Journal of Complex Networks 2(3) 313-354 (2018)

doi:10.1093/comnet/cnu004

\title{
On Global Stability of Financial Networks
}

\author{
BHASKAR DASGUPTA* \\ Department of Computer Science, University of Illinois at Chicago, Chicago, IL 60607, USA \\ ${ }^{*}$ Corresponding author: bdasgup@uic.edu \\ AND \\ LAKSHMI KALIGOUNDER \\ Department of Computer Science, University of Illinois at Chicago, Chicago, IL 60607, USA
}

[Received November 2, 2013; Accepted January 23, 2014]

\begin{abstract}
Recent crisis in the global financial world has generated renewed interests in fragilities of global financial networks among economists and regulatory authorities. In particular, a potential vulnerability of the financial networks is the "financial contagion" process in which insolvencies of individual entities propagate through the "web of dependencies" to affect the entire system. In this paper, we formalize an extension of a financial network model originally proposed by Nier et al. for scenarios such as the over-the-counter derivatives market, define a suitable global stability measure for this model, and perform a comprehensive evaluation of this stability measure over more than 700,000 combinations of networks types and parameter combinations. Based on our evaluations, we discover many interesting implications of our evaluations of this stability measure, and derive topological properties and parameter combinations that may be used to flag the network as a possible fragile network. An interactive software FIN-STAB for computing the stability is available from the website www2.cs.uic.edu/ dasgupta/financial-simulator-files.
\end{abstract}

Keywords: Financial networks, financial contagion, global stability.

2000 Math Subject Classification: 91B55, 91G99, 05C82.

\section{Introduction}

Recent unprecedented level of global financial crisis has clearly exposed potential weaknesses of the global economic system, renewing interests in the determination of fragilities of various segments of the global economy. Since financial institutions governed by borrowing, lending and participation in risky investments played a crucial role in this crisis, they have attracted a major part of the attention of economists; see [35] for a survey. The issue of instability of free market based financial systems is not new and has been under discussion among the economists starting with the early works of [30, 42] during the 1930's great depression era. However, the exact causes of such instabilities have not been unanimously agreed upon yet. Economists such as Ekelund and Thornton [27] contend that a major reason for the recent financial crisis is the enactment of an act that removed several restrictions on mixing investment and consumer banking, whereas other economists such as Calabria disagree with such an assertion [14]. Some economists such as Minsky have argued that such instabilities are are systemic for many modern market-based economic systems [52].

One motivation in this paper to investigate global stabilities of financial networks comes from the point of view of a regulatory agency (as was also the case, for example, in [35]). A regulatory agency with sufficient knowledge about a part of a global financial network is expected to periodically evaluate the stability of the network, and flag the network ex ante for further analysis if it fails some preliminary 


\section{DASGUPTA AND KALIGOUNDER}

test or exceeds some minimum threshold of vulnerability. In this motivation, flagging a network as vulnerable does not necessarily imply that such is the case, but that such a network requires further analysis based on other aspects of free market economics that are not or simply cannot be modeled 1 . While too many false positives may drain the finite resources of a regulatory agency for further analysis and investigation, this motivation assumes that vulnerability is too important an issue to be left for an $e x$ post analysis.

Similar to prior research works such as [9, 25, 35, 55, 33, 49], our study of the vulnerability of financial networks also assumes the absence of government intervention as banks become insolvent. While this is an extreme worst-case situation, the main goal of such type of studies is to see if the network can survive a shock even under extreme situations. A further reason for not allowing any intervention is, unlike the case of public health issues such as controlling spread of epidemics, government intervention in a capitalist financial system is often not allowed or requires complex political and administrative operatives.

\section{Brief review of related prior works on financial networks}

Although there is a large amount of literature on stability of financial systems (e.g., see [25, 9, 16, 6, 1, $26,43,51,45,3,24,33,31,34,47,23,46,38,55,22,7])$, very few prior papers have mathematically defined a global stability measure and performed a comprehensive evaluation of such a measure as done in this paper. A most recent research work related to our work is the paper by Minoiu and Reyes [51] in which the authors analyzed global banking networks data on cross-border banking flows for 184 countries during 1978-2010 using local connectivity and clustering measures. Below we review other related prior research works. Although ordinarily one would expect the risk of contagion to be larger in a highly interconnected banking system, prior simulation works indicate that higher connectivity among banks may sometimes lead to lower risk of contagion. Due to the large volume of prior research works, we are only able to review a selected subset of related prior research works, leaving many other exciting research results in the bibliographies of the cited papers.

Allen and Gale [3] found that when consumers have the liquidity preferences as introduced by Diamond and Dybvig [24] and have random liquidity needs, banks perfectly insure against liquidity fluctuations by exchanging interbank deposits, but the connections created by swapping deposits expose the entire system to contagion. Based on such studies, Allen and Gale [3] concluded that incomplete networks are more prone to contagion than networks with maximum connectivity since better-connected networks are more resilient via transfer of proportion of the losses in one bank's portfolio to more banks through interbank agreements. On the other hand, Gai and Kapadia [33] argued that the higher is the connectivity among banks the more will be the contagion effect during crisis. Freixas et al. [31] explored the case of banks that face liquidity fluctuations due to the uncertainty about consumers withdrawing funds. Haldane [34] suggested that contagion should be measured based on the interconnectedness of each institution within the financial system. Liedorp et al. [47] argued that both large lending and borrowing shares in interbank markets increase the riskiness of banks active in the dutch banking market.

Dasgupta [23] explored how linkages between banks, represented by cross-holding of deposits, can be a source of contagious breakdowns by investigating how depositors, who receive a private signal about fundamentals of banks, may want to withdraw their deposits if they believe that enough other

\footnotetext{
${ }^{1}$ For example, some such factors are the rumors and panics caused by the insolvency of a large bank and a possible subsequent credit freeze. While fears, panics and rumors are all real aspects in networked economics, there are hardly any universally agreed upon good way of modelling them.
} 


\section{STABILITY OF FINANCIAL NETWORKS}

depositors will do the same. Lagunoff and Schreft [46] studies a network model in which the return on an agents' portfolio depends on the portfolio allocations of other agents. Iazzetta and Manna [38] used network topology analysis on monthly data on deposits exchange to gain more insight into the way a liquidity crisis spreads. Nier et al. [55] explored the dependency of systemic risks on the structure of the banking system and the resilience (or lack thereof) of such a system to contagious defaults via graph theoretic approach. Corbo and Demange [22] explored the relationship of the structure of interbank connections to the contagion risk of defaults given the exogenous default of set of banks. Babus (7) studied how the trade-off between the benefits and the costs of being linked changes depending on the network structure, and observed that, when the network is maximal, liquidity can be redistributed in the system to make the risk of contagion minimal.

Acemoglu et al. [1] and Zawadowski [64] do investigate stability of financial networks, but differently from our study. Both [1] and [64] consider two specific network topologies, namely the ring topology and the complete network topology, as opposed to a more general class of topologies in our study. Both [1] and [64] consider only the effect of the shock propagation for a few discrete time steps, as opposed to our study; in the terminology of [9], this can be thought of as a "violent death" of the network as opposed to the "slow poisoning death" that our paper investigates. The model and structure/terms of bilateral interbank agreements in [1], namely that banks lend to one another through debt contracts with contingency covenants, is quite different from ours. As a results, the conclusions in [1, 64] do not directly apply to our model and the corresponding simulation environment.

Attribute propagation models have been investigated in the past in other contexts such as influence maximization in social networks [41, 18, 17, 13], disease spreading in urban networks [29, 19, 28], and percolation models in physics and mathematics [61]. However, the shock propagation model in this paper is very different from all these models. For example:

- Almost all of the other models include a trivial solution in which the attribute spreads to the entire network if we inject each node individually with the attribute. This is not the case with the shock propagation model.

- If shocking a subset of nodes makes $x$ nodes in the network fail, then adding more nodes to this subset may not necessarily lead to the failure of $x$ or more than $x$ nodes of the network.

- The complexity of many previous attribute propagation models arises due to the presence of cycles in the graph. In contrast, the shock propagation model may be highly complex even when the given network is acyclic. Instead, a key component of the complexity arises due to two or more directed paths sharing a node.

\section{Organization of the paper}

The rest of the paper is organized as follows:

- In Section 5 we describe the network model and the corresponding stability measure. In particular:

- In Section 5.1 we define the balance sheet equations, and the two (homogeneous and heterogeneous) versions of our model that provide an appropriate formalization and extension of the basic prior model of [25, 55].

- In Sections 5.2 5.3, we provide formalizations of how the initial failures of some nodes in the network (i.e., a shock) originate, and how such failures are propagated to other network nodes in successive time steps using a discrete-time shock propagation equation. 
DASGUPTA AND KALIGOUNDER

- In Section 5.5 we define our global network stability measure $\mathscr{K}$.

- In Sections 5.4 and 5.6 we provide rationales for the network model and the global stability measure, respectively.

- In Section 6 we describe our simulation environment and the combinations of parameters that are being explored. In particular:

- In Section 6.1, we discuss the random network models for generation of network topologies.

- In Section 6.2 we state and justify the two modes of initial failures (idiosyncratic and coordinated) that are being used in the simulation.

- In Sections 6.3 6.4, we describe the combinations of parameters used for homogeneous and heterogeneous networks and few other minor details of the simulation environment.

- In Section 7 we discuss our findings from the evaluation of the stabilities of the networks. In particular:

- Our six conclusions (1)_6 for the stability measure involving various combinations of network topology and parameters appear in Sections 7.17 .4

- In Section 7.5 we discuss two phase transition properties of the stability measure with an intuitive explanation for one of them.

Though the issue of stability of financial systems has been discussed by prior researchers [25, 9, 16, 6, 1 , $26,43,51,45,3,24,33,31,34,47,23,46,38,55,22,77$, no prior paper has performed a comprehensive evaluation of a global stability measure as done in this paper.

\section{Economic policy implications}

Returning to our original motivation of flagging financial networks for potential vulnerabilities, our results suggest that a network model similar to that used in the paper may be flagged for the following cases:

- the equity to asset ratios of most banks are low,

- the network has a highly skewed distribution of external assets and inter-bank exposures among its banks and the network is sufficiently sparse,

- the network does not have either a highly skewed distribution of external assets or a highly skewed distribution of inter-bank exposures among its banks, but the network is sufficiently dense.

\section{Our financial network model and stability measure}

Since our model has a large number of parameters, for the benefit of the reader we have included a short definition for major parameters at the beginning of each subsection where they are used. 


\section{STABILITY OF FINANCIAL NETWORKS}

\begin{tabular}{c|l|l}
\hline \multicolumn{3}{c}{ A list of major parameters used in this section } \\
\hline $\begin{array}{c}\mathscr{E} \text { total external asset } \\
=\end{array} \quad \mathscr{I}$ total inter-bank exposure & $\gamma$ ratio of equity to asset \\
$w(u, v) \quad e=(u, v)$ & $\boldsymbol{l}_{v}$ interbank asset & $\begin{array}{c}\text { effective share of } \\
\text { total external asset }\end{array}$ \\
\hline$a_{v}$ total asset & $b_{v}$ total interbank borrowing & $c_{v}$ net worth (equity) \\
\hline
\end{tabular}

\section{$5.1 \quad$ Network model and balance sheet}

We state a formalization of an ex ante financial network model similar to what has been used by researchers from Bank of England and elsewhere [25, 35, 55, 33, 49]. As was done by these prior researchers, we formulate our model in terms of balance-sheet "insolvency cascades" in a network of financial institutions (hereafter simply called "banks" and "banking networks") with interlinked balance sheets, where losses flow into the asset side of the balance sheets. The same formulation can be used to analyze cascades of cash-flow insolvency in over-the-counter derivatives markets. From now on, we will refer to balance-sheet insolvency simply as insolvency.

The banking network is represented by a parameterized node-weighted and edge-weighted directed graph $G=(V, E, \Gamma)$ in the following mannert:

- $\Gamma=\{\mathscr{E}, \mathscr{I}, \gamma\}$ is the set of parameters where

- $\mathscr{E} \in \mathbb{R}$ is the total external asset,

- $\mathscr{I} \in \mathbb{R}$ is the total inter-bank exposure,

- $A=\mathscr{I}+\mathscr{E}$ is the total asset, and

$-\gamma \in(0,1)$ is the ratio of equity to asset.

- $V$ is the set of $n$ banks where

- the node weight $\sigma_{v} \in[0,1]$ denotes the share of total external asset for each bank $v \in V$ $\left(\sum_{v} \sigma_{v}=1\right)$.

- $E$ represents the set of $m$ direct inter-bank exposures where

- $w(e)=w(u, v)>0$ is the weight of a directed edge $e=(u, v) \in E$.

The (interlocked) balance sheet for each node (bank) $v \in V$ is shown in Table 1 Two types of banking network models are considered:

Homogeneous model: $\mathscr{E}$ and $\mathscr{I}$ are equally distributed among the nodes and the edges, respectively, i.e., $\sigma_{v}=\frac{1}{n}$ for every node $v$, and $w(e)=\frac{\mathscr{I}}{m}$ for every edge $e$.

Heterogeneous model: $\mathscr{E}$ and $\mathscr{I}$ are not necessarily equally distributed among the nodes and the edges, respectively.

Both homogeneous and heterogeneous network models are relevant in practice, and have been investigated by prior researchers such as $[9,35,55,37,33,49]$.

\footnotetext{
${ }^{2}$ The parameters $\mathscr{E}, \mathscr{I}, A, \gamma$ and $\Phi$ were also used by prior researchers, and the parameters $\sigma_{v}$ and $w(e)$ are generalizations of parameters used by prior researchers.
} 
DASGUPTA AND KALIGOUNDER

Table 1. Relevant balance sheet details of a node $v$ in the network [25, 55, 9]. The total amount of external assets $\mathscr{E}$ is assumed to be large enough such that $b_{v}-\imath_{v}+\sigma_{v} \mathscr{E}$ is positive.

\begin{tabular}{|c|c|c|c|}
\hline \multicolumn{2}{|c|}{ Assets } & \multicolumn{2}{|c|}{ Liabilities } \\
\hline $\boldsymbol{u}_{v}=\sum_{(v, u) \in E} w(v, u)$ & interbank asset & & \\
\hline$e_{v}=b_{v}-u_{v}+\sigma_{v} \mathscr{E}$ & $\begin{array}{l}\text { effective share of } \\
\text { total external asset }\end{array}$ & $b_{v}=\sum_{(u, v) \in E} w(u, v)$ & $\begin{array}{l}\text { total interbank } \\
\text { borrowing }\end{array}$ \\
\hline$a_{v}=b_{v}+\sigma_{v} \mathscr{E}$ & total asset & & \\
\hline
\end{tabular}

\subsection{Initial insolvency via shocks}

\begin{tabular}{ll|l|l}
\hline \multicolumn{2}{c}{ A list of major parameters and definitions used in this section } \\
\hline$V_{\boldsymbol{*}}$ & set of initially shocked nodes & $\Phi$ severity of initial shock $t$ time variable \\
\hline shocking mechanism: & $\begin{array}{l}\text { rule to select an initial subset } \\
\text { of nodes to be shocked }\end{array}$ \\
\hline
\end{tabular}

The initial insolvencies of a banking network at time $t=0$ are caused by "shocks" received by a subset $\emptyset \subset V \mathbf{x} \subseteq V$ of nodes. Such shocks can occur, for example, due to operational risks (e.g., fraud 3 ) or credit risks, and has the effect of reducing the external assets of an selected subset of banks. Mathematically, the effect of the initial shock is to simultaneously decrease the external assets of each shocked node $v \in V_{\mathbf{X}}$ from $e_{v}$ by $s_{v}=\Phi e_{v}$, thereby reducing the net worth of $v$ from its original value $c_{v}$ to $c_{v}-s_{v}$, where $(0,1] \ni \Phi>\gamma$ is a parameter denoting the severity of the initial shock.

In the rest of the paper, by the phrase "shocking mechanism", we refer to the rule that is used to select the initial subset of nodes to be shocked5.

\subsection{Insolvency propagation equation}

\begin{tabular}{l|ll}
\hline \multicolumn{3}{c}{ A list of major parameters used in this section } \\
\hline $\operatorname{deg}^{\text {in }}(v)$ in-degree of node $v$ & $V_{\&<}\left(t, V_{\mathbf{*}}\right)$ & $\begin{array}{l}\text { set of nodes that became insolvent before time } t \\
\text { when initial shock is provided to nodes in } V_{\boldsymbol{*}}\end{array}$ \\
\hline
\end{tabular}

Let the notation deg in $(v)$ denote the in-degree of node $v$. The insolvencies propagate in discrete time units $t=0,1,2, \ldots$; we add " $\left(\ldots, t, V_{\mathbf{x}}, \ldots\right)$ " to all relevant variables to indicate their dependences on $t$ and on the set $V_{\mathbf{x}}$ of initially shocked nodes. A bank becomes insolvent if its modified net worth becomes negative, and such a bank is removed from the network in the next time step. Let $V_{8<}\left(t, V_{\mathbf{x}}\right) \subseteq V$ denote the set of nodes that became insolvent before time $t$ when an initial shock is provided to the nodes 6 in $V_{\mathbf{x}}$. The insolvencies of banks at time $t$ affect the equity of other banks in the network at the next time

${ }^{3}$ Iyer and Peydro [39] show that fraud is an important cause of bank insolvency.

${ }^{4}$ Nier et al. [55] considered shocking only one (or a few) bank and empirically studying the effect of the shock on the entire network. Berman et al. [9], on the other hand, analyzed the computational complexity issues of the problem of selecting a subset of nodes such that shocking them will make the network fail.

${ }^{5}$ Shocking mechanisms were not formally defined by prior researchers, but it was often implicit in their discussions.

${ }^{6}$ Thus, in particular, deg ${ }^{\text {in }}\left(v, t, V_{\mathbf{*}}\right)$ is the in-degree in the graph induced by the nodes in $V \backslash V_{\mathrm{S}<}\left(t, V_{\mathbf{x}}\right)$. 


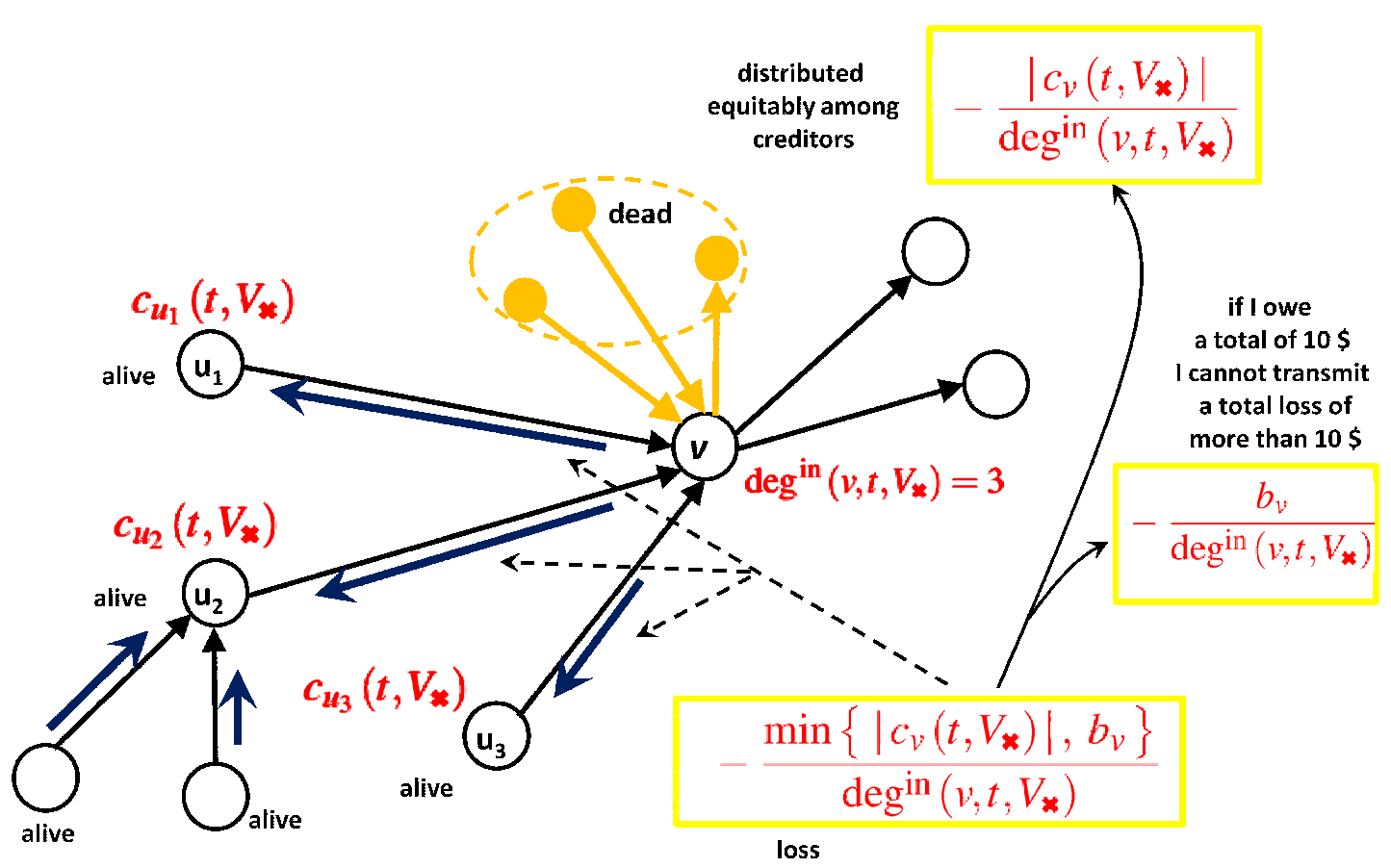

FIG. 1. Pictorial illustration of the shock transmission equation for a node $v$ from time $t$ to time $t+1$.

step $t+1$ by the following non-linear "insolvency propagation equation" 개:

$$
\begin{aligned}
\forall u \in V \backslash V_{8<}\left(t, V_{\mathbf{x}}\right): c_{u}\left(t+1, V_{\mathbf{x}}\right)=c_{u}\left(t, V_{\mathbf{x}}\right) & -\sum_{\left(c_{v}\left(t, V_{\mathbf{x}}\right)<0\right)} \frac{\min \left\{\left|c_{v}\left(t, V_{\mathbf{x}}\right)\right|, b_{v}\right\}}{\operatorname{deg} \operatorname{in}\left(v, t, V_{\mathbf{x}}\right)} \\
v: & \wedge\left(v \in V \backslash V_{s<}\left(t, V_{\mathbf{x}}\right)\right) \\
& \wedge((u, v) \in E)
\end{aligned}
$$

In Equation (5.1), the term $\frac{\left|c_{v}\left(t, V_{\mathbf{x}}\right)\right|}{\operatorname{deg}^{\text {in }}\left(v, t, V_{\mathbf{x}}\right)}$ ensures the loss of equity of an insolvent bank to be distributed equitably among its creditors that have not become insolvent yet, whereas the term $\frac{b_{v}}{\operatorname{deg}^{\text {in }}\left(v, t, V_{\mathbf{x}}\right)}$ ensures that the total loss propagated cannot be more than the total interbank exposure of the insolvent bank; see FIG. 1 for a pictorial illustration. The insolvency propagation continues until no new bank becomes insolvent. For notational convenience, we may use $(\ldots, t, \ldots)$ instead of $\left(\ldots, t, V_{\mathbf{x}}, \ldots\right)$ when $V_{\mathbf{x}}$ is clear from the context or is irrelevant.

\footnotetext{
${ }^{7}$ An equation of same flavor with some simplification and omitted details was described in words by Nier et al. [55] and Eboli [25].

${ }^{8}$ Equation [5.1] is highly non-linear. The results in [9] indicate that in general it is NP-hard to find a subset $V_{\mathbf{x}}$ of initially shocked nodes such that $\left|\lim _{t \rightarrow \infty} V_{\mathcal{S}<}\left(t, V_{\mathbf{x}}\right)\right|$ is exactly or approximately maximized.
} 


\section{DASGUPTA AND KALIGOUNDER}

\subsection{Rationale for the network model and insolvency propagation equation}

As prior researchers [55, 2, 43] have commented:

"conceptual frameworks from the theory of weighted graphs with additional parameters may provide a powerful tool for analysis of banking network models".

Several parametric graph-theoretic models, differing in the way edges are interpreted and additional parameters are used to characterize the contagion, have been used by prior researchers in finance and banking industry to study various research questions involving financial systems [9, 25, 35, 37, 55, 32, 63, 53, 5, 21, 62, 64]. As noted by researchers in [55, 5]:

"the modelling challenge in studying banking networks lies not so much in analyzing a model that is flexible enough to represent all types of insolvency cascades, but in studying a model that can mimic the empirical properties of these different types of networks".

The insolvency propagation model formalized and evaluated in this paper using a mathematically precise abstraction is similar to or a generalization of the models in [9, 25, 35, 55, 37, 33, 49, 5] that represent cascades of cash-flow insolvencies. As [5] observes, over-the-counter (OTC) derivatives and similar markets are prone to this type of cascades. In such markets parties deal directly with one another rather than passing through an exchange, and thus each party is subject to the risk that the other party does not fulfill its payment obligations. The following example from [5] illustrates chains of such interactions:

[5] “Consider two parties $A$ and $B$, such that $A$ has a receivable from party $B$ upon the realization of some event. If $B$ does not dispose of enough liquid reserves, it will default on the payment. Now consider that $B$ has entered an off-setting contract with another party $C$, hedging its exposure to the random event. If $C$ is cash-flow solvent, then the payment will flow through the intermediary $B$ and reach $A$. However, if $C$ is cash-flow insolvent and defaults, then the intermediary $B$ might become cash-flow insolvent if it depends on receivables from $C$ to meet its payment obligations to $A$ ”.

The length of such chains of interactions in some OTC markets, like the credit default swap market, is significant [20, 50], thereby increasing the probability of cascade of cash-flow insolvencies [2]. As [55] observes, an insolvency propagation model such as the one studied here

"conceptualises the main characteristics of a financial system using network theory by relating the cascading behavior of financial networks both to the local properties of the nodes and to the underlying topology of the network, allowing us to vary continuously the key parameters of the network".

Although the cascading effect studied is of somewhat special and simplified nature, as noted by [35]:

"This is a deliberate oversimplification, aimed at a clearer understanding of how an initial failure can propagate shocks throughout the system".

\subsection{A measure of global stability}

Consider a banking network model as described in Sections 5.1 5.3 . Let $\mathscr{K} \in(0,1]$ be a real number 10 denoting the fraction of nodes in $V$ that received the initial shock under a shocking mechanism $Y$ and

\footnotetext{
${ }^{9}$ A mathematically precise definition of global stability measure was omitted by most prior researchers.

${ }^{10} \mathscr{K}$ is a new parameter not used by prior researchers.
} 


\section{STABILITY OF FINANCIAL NETWORKS}

\begin{tabular}{l|ll}
\hline \multicolumn{2}{|c}{ A list of major parameters used in this section } \\
\hline$\Upsilon$ a shocking mechanism & $\begin{array}{l}\text { on an average 100x\% nodes of the network become } \\
\text { insolvent with the given values of } \gamma \text { and } \Phi \text { if we pro- } \\
\text { vide an initial shock to a random subset of } 100 \mathscr{K} \% \text { of } \\
\text { nodes selected using the shocking mechanism } \Upsilon\end{array}$ \\
\hline
\end{tabular}

let $S_{r, \mathscr{K}}$ be the set of all possible $(\mathscr{K} n)$-element subsets of $V$. The vulnerability index 11 of the network is then defined as 12

$$
\xi(\mathscr{K}, G, \gamma, \Phi, \Upsilon)=\frac{1}{n} \times \mathbb{E}\left[\left|\lim _{t \rightarrow \infty} V_{\mathscr{S}<}\left(t, V_{\boldsymbol{*}}\right)\right|: V_{\mathbf{*}} \text { is selected randomly from } S_{\Upsilon, \mathscr{K}}\right]
$$

In the above definition, the $\frac{1}{n}$ factor is only for a min-max normalization [36] to ensure that $0 \leqslant$ $\xi(\mathscr{K}, G, \gamma, \Phi, \Upsilon) \leqslant 1$. Noting that no new node in the network may fail at a time $t \geqslant n$, we may simplify the above expression for $\xi$ as:

$$
\begin{aligned}
\xi(\mathscr{K}, G, \gamma, \Phi, \Upsilon)=\frac{1}{n} \times \mathbb{E}\left[\left|V_{\mathcal{S}}\left(n, V_{\mathbf{x}}\right)\right|: V_{\boldsymbol{*}} \text { is selected randomly from } S_{\Upsilon, \mathscr{K}}\right] \\
\Longrightarrow \operatorname{Pr}\left[\left|V_{\mathcal{S}}\left(n, V_{\boldsymbol{*}}\right)\right| \geqslant n \xi(\mathscr{K}, G, \gamma, \Phi, \Upsilon): V_{\boldsymbol{*}} \text { is selected randomly from } S_{\Upsilon, \mathscr{K}}\right]>0
\end{aligned}
$$

As an example, $\xi(0.1, G, 0.3,0.5$, random $)=0.9$ means that with positive probability $90 \%$ nodes of the network $G$ become insolvent with $\gamma=0.3$ and $\Phi=0.5$ if we provide an initial shock to a random subset of $10 \%$ of nodes of $G$. Note that lower values of $\xi$ imply higher global stability of a network. For simplicity, we may omit the arguments of $\xi$ when they are clear from the context. A pseudo-code for calculating $\xi$ is shown in Fig. 2

\subsection{Rationale for the global stability measure}

It is possible to think of other alternate measures of global stability than the one quantified above. However, the measure introduced above is in tune with the ideas that references [25, 55, 35, 37] directly (and, some other references such as [32, 63, 49] implicitly) used to empirically study their networks. Thus, in formalizing our global stability measure, we have decided to follow the cue provided by other researchers in the banking industry who have studied various insolvency propagation models. Measures of similar flavor have also been used by prior researchers in social networks in the context of influence maximization [17, 18].

\section{Simulation environment and explored parameter space}

In Table 2 we provide a summary of our simulation environment and explored parameter space. Individual components of the summary are discussed in Sections 6.1 6.4

\footnotetext{
${ }^{11}$ Although simple topological properties such as clustering coefficients have been used by authors to study properties of networks [56, 60], they are too simplistic for stability analysis of financial networks.

${ }^{12}$ In this definition, we implicitly assume that the shocking mechanism $\Upsilon$ allows one to select at least one set of $\mathscr{K} n$ nodes for the initial shock. Otherwise, we define $\xi$ to be zero.
} 


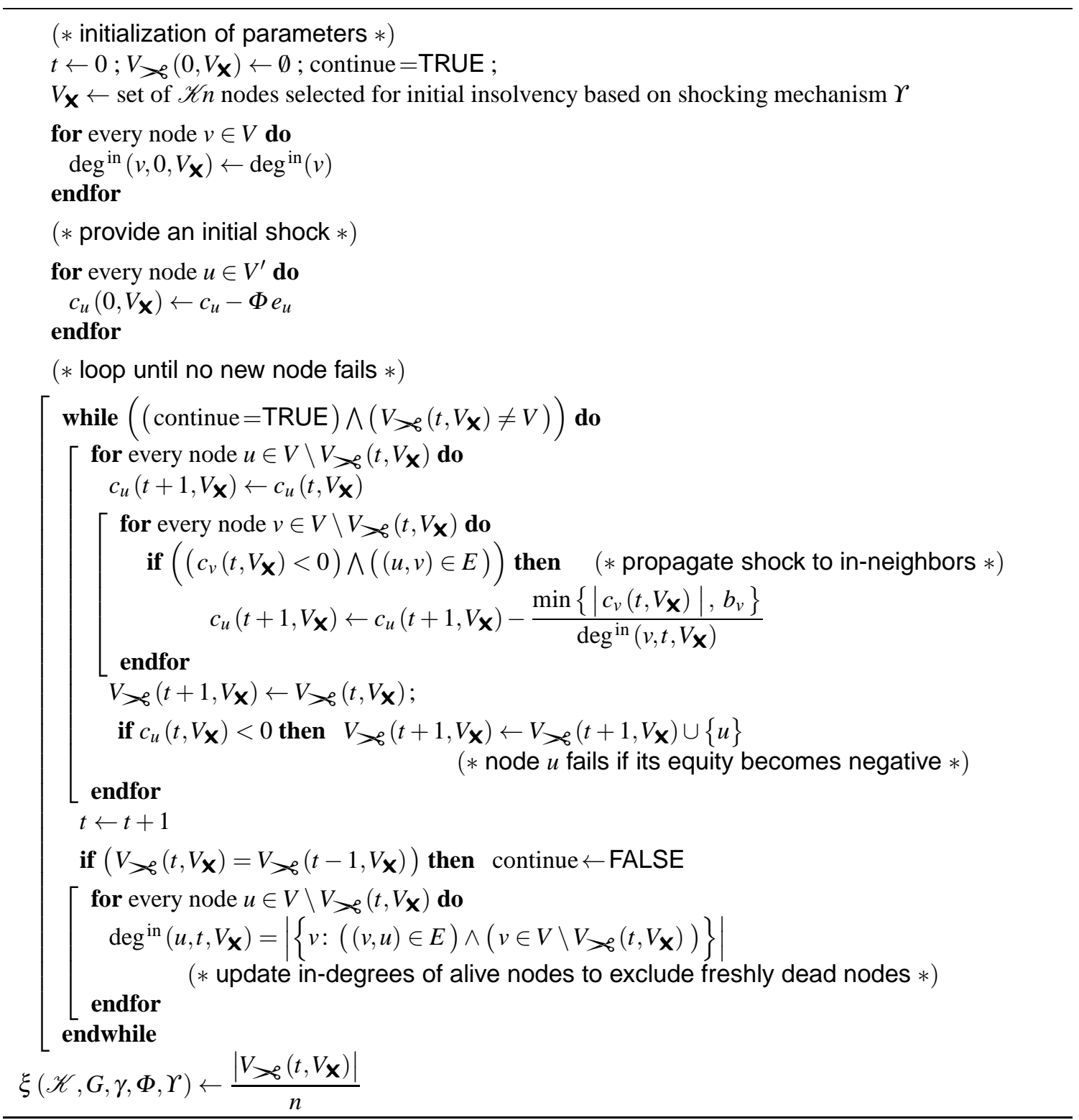

FIG. 2. Pseudo-code for calculating $\xi(\mathscr{K}, G, \gamma, \Phi, \Upsilon)$. Comments in the pseudo-code are enclosed by (* and $*)$. An implementation of the pseudo-code is available at www2.cs.uic.edu/ dasgupta/financial-simulator-files 
STABILITY OF FINANCIAL NETWORKS

\begin{tabular}{|c|c|c|c|}
\hline parameter & \multicolumn{2}{|c|}{ explored values for the parameter } & \multirow{11}{*}{$\begin{array}{c}\text { total } \\
\text { number of } \\
\text { parameter } \\
\text { combinations } \\
>700,000\end{array}$} \\
\hline \multirow{2}{*}{ network type } & \multicolumn{2}{|l|}{ homogeneous } & \\
\hline & $(\alpha, \beta)$-heterogeneous & $\begin{array}{l}\alpha=0.1, \beta=0.95 \\
\alpha=0.2, \beta=0.6\end{array}$ & \\
\hline \multirow[t]{2}{*}{ network topology } & directed scale-free & $\begin{array}{l}\text { age degree } 1 \text { (in-arborescence) } \\
\text { average degree } 3 \\
\text { average degree } 6\end{array}$ & \\
\hline & directed Erdös-Rényi & $\begin{array}{l}\text { average degree } 3 \\
\text { average degree } 6\end{array}$ & \\
\hline shocking mechanism & \multicolumn{2}{|c|}{ idiosyncratic, coordinated } & \\
\hline number of nodes & \multicolumn{2}{|l|}{$50,100,300$} & \\
\hline$E / I$ & \multicolumn{2}{|c|}{$0.25,0.5,0.75,1,1.25,1.5,1.75,2,2.25,2.5,2.75,3,3.25,3.5$} & \\
\hline$\Phi$ & \multicolumn{2}{|l|}{$0.5,0.6,0.7,0.8,0.9$} & \\
\hline $\mathscr{K}$ & \multicolumn{2}{|c|}{$0.1,0.2,0.3,0.4,0.5,0.6,0.7,0.8,0.9$} & \\
\hline$\gamma$ & \multicolumn{2}{|c|}{$0.05,0.1,0.15, \ldots, \Phi-0.05$} & \\
\hline
\end{tabular}

Table 2. A summary of simulation environment and explored parameter space.

\section{$6.1 \quad$ Network topology 13}

We consider two topology models previously used by economists to generate random financial networks:

- the directed scale-free (SF) network model [8] that has been used by prior financial network researchers such as [59, 54, 5, 21], and

- the directed Erdös-Rényi (ER) network model [11] that has been used by prior financial network researchers such as [58, 33, 48, 22, 15].

Generation of directed ER networks is computationally trivial: given a value $0<p<1$ that parameterizes the ER network, for every ordered pair of distinct nodes $(u, v)$ we let $\operatorname{Pr}[(u, v) \in E]=1 / p$. Letting $p=d / n$ generates a random ER network whose average degree is $d$ with high probability.

The directed SF networks in this paper are generated using the algorithm outlined by Bollobas et al. [11]. The algorithm works as follows. Let $a, b, \eta, \delta_{\text {in }}$ (in-degree) and $\delta_{\text {out }}$ (out-

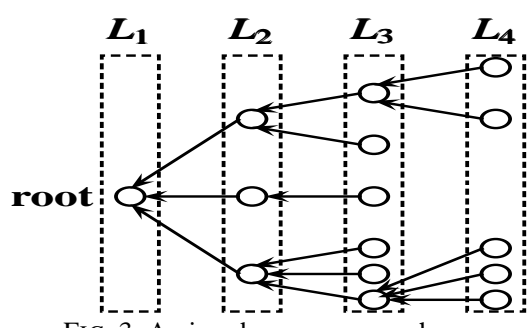

FIG. 3. An in-arborescence graph. degree) be non-negative real numbers with $a+b+\eta=1$. The initial graph $G(0)$ at step $\ell=0$ has just one node with no edges. At step $\ell>0$ the graph $G(\ell)$ has exactly $\ell$ edges and a random number $n_{\ell}$ of nodes. For $\ell \geqslant 0, G(\ell+1)$ is obtained from $G(\ell)$ by using the following rules:

\footnotetext{
${ }^{13}$ One may obviously ask: why not use "real" networks? There are several obstacles however that make this desirable goal impossible to achieve. For example: $(\boldsymbol{a})$ Due to their highly sensitive nature, such networks with all relevant parameters are rarely publicly available. (b) For the kind of inferences that we make in this paper, we need hundreds of thousands of large networks to have any statistical validity (in this paper, we explore more than 700,000 networks).
} 


\section{DASGUPTA AND KALIGOUNDER}

- With probability $a$, add a new node $v$ together with an edge from $v$ to an existing node $w$, where $w$ is chosen randomly such that

$$
\operatorname{Pr}[w=u]=\frac{\left(d_{\text {in }}(u)+\delta_{\text {in }}\right)}{\left(\ell+\delta_{\text {in }} n_{\ell}\right)}
$$

for every existing node $u$, where $d_{\text {in }}(u)$ is the in-degree of node $u$ in $G(\ell)$.

- With probability $b$, add an edge from an existing node $v$ to an existing node $w$, where $v$ and $w$ are chosen independently, such that

$$
\begin{gathered}
\operatorname{Pr}[v=u]=\frac{d_{\text {out }}(u)+\delta_{\text {out }}}{\ell+\delta_{\text {out }} n_{\ell}} \text { for every existing node } u \\
\operatorname{Pr}[w=u]=\frac{d_{\text {in }}(u)+\delta_{\text {in }}}{\ell+\delta_{\text {in }} n_{\ell}} \text { for every existing node } u
\end{gathered}
$$

where $d_{\text {out }}(u)$ is the out-degree of node $u$ in $G(\ell)$.

- With probability $\eta$, add a new node $w$ and an edge from an existing node $v$ to $w$, where $v$ is chosen such that $\operatorname{Pr}[v=u]=\frac{d_{\text {out }}(u)+\delta_{\text {out }}}{\ell+\delta_{\text {out }} n_{\ell}}$ for every existing node $u$.

To study the effect of connectivity on network stability, we generated random SF and ER networks with average degree 14 of 3 and 6.

In addition, to study the effect of sparse hierarchical topology on network stability, we used the Barábasi-Albert preferential-attachment model [8] to generate random in-arborescence networks. Inarborescences are directed rooted trees with all edges oriented towards the root (see Fig. 3), and have the following well-known topological properties:

- They belong to the class of sparsest connected directed acyclic graphs.

- They are hierarchical networks, i.e., the nodes can be partitioned into levels $L_{1}, L_{2}, \ldots, L_{p}$ such that $L_{1}$ has exactly one node (the "root") and nodes in any level $L_{i}$ have directed edges only to nodes in $L_{i-1}$ (see Fig. 3). The root may model a "central bank" that lends to other banks but does not borrow from any bank.

The algorithm for generating a random in-arborescence network $G$ using the preferential-attachment model [8] is as follows:

- Initialize $G=(V, E)$ to have one node (the root) and no edges.

- Repeat the following steps till $G$ has $n$ nodes:

- Randomly select a node $u$ in $G$ such that, for every node $v$ in $G, \operatorname{Pr}[u=v]=\frac{\operatorname{deg}(v)}{\sum_{w \in V} \operatorname{deg}(w)}$ where $\operatorname{deg}(y)$ denotes the degree of node $y$ in $G$.

- Add a new node $x$ and an undirected edge $\{x, u\}$ in $G$.

- Orient all the edges towards the root.

${ }^{14}$ There are many ways to fix the parameters to get the desired average degree. For example, as observed in [11], letting $\delta_{\text {out }}=0$ and $\alpha>0$, one obtains $\mathbb{E}[$ number of nodes in $G(t)$ of in-degree $x] \propto x^{-\left(1+\frac{1+\delta_{\text {in }}(\alpha+\eta)}{\alpha+\beta}\right)} t$ and $\mathbb{E}[$ number of nodes in $G(t)$ of out-degree $x] \propto x^{-\left(\frac{2-\alpha}{1-\alpha}\right)} t$. 


\section{STABILITY OF FINANCIAL NETWORKS}

\subsection{Shocking mechanisms $\Upsilon$}

Recall that a shocking mechanism $\Upsilon$ provides a rule to select the initial subset of nodes to be shocked. The following two mechanisms are used to select the nodes to receive the initial shock.

Idiosyncratic (random) shocking mechanism We select a subset of nodes uniformly at random. This corresponds to random idiosyncratic initial insolvencies of banks, and is a choice that has been used by prior researchers such as [55, 37, 35, 33, 49].

Coordinated shocking mechanism 15 . In this type of non-idiosyncratic correlated shocking mechanism, we seek to play an adversarial role 16 in selecting nodes for the initial shock that may cause more damage to the stability of the network. The selection of an adversarial strategy depends on whether the network is homogeneous or heterogeneous. The coordinated shocking mechanism falls under the general category of correlated shocks where the nodes with high (weighted) in-degrees are correlated.

For homogeneous networks, recall that all nodes have the same share of the total external asset $\mathscr{E}$. However, the total interbank exposure $b_{v}$ of a node $v$ is directly proportional to the in-degree of $v$, and, as per Equation (5.1), nodes with higher inter-bank exposures are more likely to transmit the shock to a larger number of other nodes. Thus, we play an adversarial role by selecting a set of $\mathscr{K} n$ nodes in non-increasing order of their in-degrees starting from a node with the highest in-degree.

For heterogeneous networks, nodes with higher "weighted" in-degrees (i.e., with higher values of the sum of weights of incoming edges) represent nodes that have larger external assets than other nodes, and have more inter-bank exposures. Thus, for heterogeneous networks we play an adversarial role by selecting $\mathscr{K} n$ nodes in non-increasing order of their weighted in-degrees starting from a node with the highest weighted in-degree.

\subsection{Network type: $(\alpha, \beta)$-heterogeneous networks}

Recall that in a heterogeneous network it is possible to have a few banks whose external assets or interbank exposures are significantly larger than the rest of the banks, i.e., it is possible to have a few banks that are "too big", and thus heterogeneous networks permit investigation of the effect of such big banks on the global stability of the entire network. To this end, we define a $(\alpha, \beta)$-heterogeneous network as follows.

DEFINITION $6.1((\boldsymbol{\alpha}, \boldsymbol{\beta})$-heterogeneous network) Let $\widetilde{V} \subseteq V$ be a random subset $V$ of $\alpha n$ nodes and let $\widetilde{E}$ be the set of edges that have at least one end-point from $\widetilde{V}$. For $0<\alpha, \beta<1$, a $(\alpha, \beta)$-heterogeneous network $G=(V, E)$ is one in which the total external and internal assets are distributed in the following manner:

\section{Distribution of $\mathscr{E}$ :}

\footnotetext{
${ }^{15}$ While correlated shocking mechanisms affecting a correlated subset of banks are relevant in practice, prior researchers such as [55, 35, 37, 33, 49] have mostly used idiosyncratic shocking mechanisms. There are at least two reasons for this. Firstly, idiosyncratic shocks are a cleaner way to study the stability of the topology of the banking network. Secondly, it is not a priori clear what kind of correlations in the shocking mechanism would lead to failure of more nodes than idiosyncratic shocks in a statistically significant way. Our coordinated shocking mechanism intuitively corresponds shocks in which banks that are "too big to fail" in terms of their assets are correlated. Our conclusion (6) shows that coordinated shocks do indeed cause more statistically significant damage to the stability of the network as opposed to random shocks.

${ }^{16}$ Usage of adversarial strategies in measuring the worst-case bounds for network properties are very common in the algorithmic literature; see, for example, see the book [12].
} 


\section{DASGUPTA AND KALIGOUNDER}

- distribute $\beta \mathscr{E}$ part of the total external asset $\mathscr{E}$ equally among the $\alpha n$ nodes in $\widetilde{V}$, and

- distribute the remaining part $(1-\beta) \mathscr{E}$ of $\mathscr{E}$ equally among the remaining $(1-$ $\alpha) n$ nodes.

\section{Distribution of $\mathscr{I}$ :}

- Distribute $\beta \mathscr{I}$ part of the total interbank exposure $\mathscr{I}$ equally among a random subset of $\alpha|\widetilde{E}|$ of edges from the edges in $\widetilde{E}$, and

- distribute the remaining part $(1-\beta) \mathscr{I}$ of $\mathscr{I}$ equally among the remaining $|E|-\alpha|\widetilde{E}|$ edges.

We performed our simulations for $(\alpha, \beta)$-heterogeneous networks for $(\alpha, \beta)=(0.1,0.95)$ and $(\alpha, \beta)=$ $(0.2,0.6)$. The combination $(\alpha, \beta)=(0.1,0.95)$ corresponds to the extreme situation in which $95 \%$ of the assets and exposures involve $10 \%$ of banks, thus creating a minority of banks that are significantly larger than the remaining banks. The other combination $(\alpha, \beta)=(0.2,0.6)$ corresponds to a less extreme situation in which there are a larger number of moderately large banks.

\subsection{Other minor details}

To correct statistical biases, for each combinations of parameters, shock types and network types, we generated 10 corresponding random networks and computed the average value of the vulnerability index over these 10 runs. For ER and SF random networks, we selected the values of network generation parameters such that the expected number of edges of the network is $3 n$ or $6 n$ depending on whether we require the average degree of the network to be 3 or 6 , respectively.

The minimum difference between two non-identical values of the average vulnerability index over 10 runs for two $n$-node networks is $1 /(10 n)$. Thus, to allow for minor statistical biases introduced by any random graph generation method, we consider two vulnerability indices to be same (within the margin of statistical error) if their absolute difference is no more than $1 /(3 n)$, which is above $1 /(10 n)$ but no more than $0.7 \%$ of the total range of the vulnerability indices.

Finally, we can assume without loss of generality that $\mathscr{I}=m$, since otherwise if $\mu=\frac{\mathscr{q}}{m} \neq 1$ then we can divide each of the quantities $\imath_{v}, b_{v}$ and $\mathscr{E}$ by $\mu$ without changing the outcome of the insolvency propagation procedure.

\section{Results}

In this section, we discuss our uncovering of many interesting relationships of the stability with other relevant parameters of the network based on our comprehensive evaluation and analysis of this stability measure. It is easy to see that there are many (at least several thousands, but significantly more in most cases) networks in the original sets of networks that are compared in two different scenarios in Tables 3/45[6]7/9[10]11 and related tables in the supplementary documents, thereby assuring the statistical validity of the comparison results.

\subsection{Effect of unequal distribution of total assets $\mathscr{E}$ and $\mathscr{I}$}

As our analysis shows, nodes with disproportionately large external assets affect the stability of the entire network in an adverse manner, and more uneven distribution of assets among nodes in the network makes the network less stable. 


\section{STABILITY OF FINANCIAL NETWORKS}

7.1.1 Effect on global stability For the same value of the common parameters $n, \frac{\mathscr{E}}{\mathscr{L}}, \mathscr{K}, \Phi$ and $\gamma$, for the same for network type (ER, SF or in-arborescence) of same average degree $(6,3$ or 1$)$ and for the same shocking mechanism $\Upsilon$ (coordinated or idiosyncratic), we compared the value of $\xi$ for the homogeneous network with the corresponding values of $\xi$ for $(0.1,0.95)$-heterogeneous and $(0.2,0.6)$ heterogeneous networks. The comparison results shown in Table 3 show most of the entries as being at least $90 \%$. Thus, we conclude:

(1) networks with all nodes having the same external assets display higher stability over similar networks with fewer nodes having disproportionately higher external assets.

Table 3. Comparison of stabilities of $(\alpha, \beta)$-heterogeneous networks with their homogeneous counter-parts over all parameter

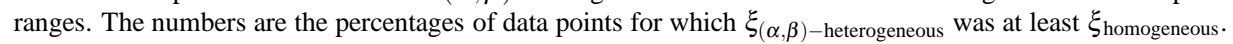

\begin{tabular}{|c|c|c|c|c|c|c|c|c|c|c|}
\hline \multirow[b]{3}{*}{$\begin{array}{l}\text { coordinated } \\
\text { shock }\end{array}$} & \multirow{2}{*}{\multicolumn{2}{|c|}{$\begin{array}{c}\text { In-arborescence } \\
\begin{array}{c}\alpha=0.1 \\
\beta=0.95\end{array} \mid \begin{array}{l}\alpha=0.2 \\
\beta=0.6\end{array}\end{array}$}} & \multicolumn{2}{|c|}{\begin{tabular}{|c|} 
ER \\
average degree 3
\end{tabular}} & \multicolumn{2}{|c|}{$\begin{array}{c}\text { ER } \\
\text { average degree } 6\end{array}$} & \multicolumn{2}{|c|}{\begin{tabular}{c|} 
SF \\
average degree 3
\end{tabular}} & \multicolumn{2}{|c|}{$\begin{array}{c}\text { SF } \\
\text { average degree } 6\end{array}$} \\
\hline & & & $\begin{array}{c}\alpha=0.1 \\
\beta=0.95\end{array}$ & $\begin{array}{l}\alpha=0.2 \\
\beta=0.6\end{array}$ & $\begin{array}{c}\alpha=0.1 \\
\beta=0.95\end{array}$ & $\left|\begin{array}{l}\alpha=0.2 \\
\beta=0.6\end{array}\right|$ & $\begin{array}{c}\alpha=0.1 \\
\beta=0.95\end{array}$ & $\mid \begin{array}{l}\alpha=0.2 \\
\beta=0.6\end{array}$ & $\begin{array}{c}\alpha=0.1 \\
\beta=0.95\end{array}$ & $\begin{array}{l}\alpha=0.2 \\
\beta=0.6\end{array}$ \\
\hline & $.91 \%$ & $\%$ & $99.26 \%$ & $98.91 \%$ & $98.46 \%$ & $98.00 \%$ & $98.22 \%$ & $91.68 \%$ & $99.13 \%$ & $97.4 \%$ \\
\hline $\begin{array}{l}\text { idiosyncratic } \\
\text { shock }\end{array}$ & $92.75 \%$ & $81.79 \%$ & $97.76 \%$ & $96.81 \%$ & $98.16 \%$ & $97.61 \%$ & $98.86 \%$ & $94.84 \%$ & $98.83 \%$ & $97.22 \%$ \\
\hline
\end{tabular}

\section{Formal intuition behind the conclusion in 1}

In spite of the highly non-linear nature of Equation (5.1), the following formal intuition may help to explain the conclusion in 1 .

LEMma 7.1 (see Section A of the appendix for a proof) Fix $\gamma, \Phi, \mathscr{E}, \mathscr{I}$ and the graph $G$. Consider any node $v \in V_{\mathbf{X}}$ and suppose that $v$ fails due to the initial shock. For every edge $(u, v) \in E$, let $\Delta_{\text {homo }}(u)$ and $\Delta_{\text {hetero }}(u)$ be the amount of shock received by node $u$ at time $t=1$ if $G$ is homogeneous or heterogeneous, respectively. Then,

$$
\mathbb{E}\left[\Delta_{\text {hetero }}(u)\right] \geqslant \frac{\beta}{\alpha} \mathbb{E}\left[\Delta_{\text {homo }}(u)\right]=\begin{aligned}
9.5 \mathbb{E}\left[\Delta_{\text {homo }}(u)\right], & \text { if }(\alpha, \beta)=(0.1,0.95) \\
3 \mathbb{E}\left[\Delta_{\text {homo }}(u)\right], & \text { if }(\alpha, \beta)=(0.2,0.6)
\end{aligned}
$$

Lemma 7.1 implies that $\mathbb{E}\left[\Delta_{\text {hetero }}(u)\right]$ is much bigger than $\mathbb{E}\left[\Delta_{\text {homo }}(u)\right]$, and thus more nodes are likely to fail beyond $t>0$ leading to a lower stability for heterogeneous networks.

7.1.2 Effect on residual instability For homogeneous networks, if the equity to asset ratio $\gamma$ is close enough to the severity of the shock $\Phi$ then the network almost always tends to be perfectly stable, as one would intuitively expect. However, the above property is not true in general for highly heterogeneous 


\section{DASGUPTA AND KALIGOUNDER}

networks in the sense that, even when $\gamma$ is close to $\Phi$, these networks (irrespective of their topologies and densities) have a minimum amount of global instability (which we term as the residual instability) 17 .

In Table 4 and supplemental Tables S1-S9 we tabulated residual instabilities for different types of homogeneous and heterogeneous networks under coordinated and idiosyncratic shocks. The numbers in these tables show, for each combination of network types, $|V|$, shocking mechanism and values of $\Phi$ and $\gamma$ such that $|\Phi-\gamma|=0.05$, the percentage of networks with this combination for which the vulnerability index $\xi$ was less than $0.05,0.1$ or 0.2 . As the reader will observe, all the numbers for heterogeneous networks are significantly lower than their homogeneous counter-parts. Thus, we conclude:

(2) a heterogeneous network, in contrast to its corresponding homogeneous version, has a residual minimum instability even if its equity to asset ratio is very large and close to the severity of the shock.

\subsection{Effect of total external assets}

A controversial belief regarding the cause of the collapse of many major financial institutions around 2007 asserts that removal of the separation between investment and consumer banking allowed a ripple effect of insolvencies of individual banks to other banks [27, 14]. In our setting, the quantity $\mathscr{E} / \mathscr{I}$ controls the total (normalized) amount of external investments of all banks in the network. Thus, varying the ratio $\mathscr{E} / \mathscr{I}$ allows us to investigate the role of the magnitude of total external investments on the stability of our banking network (see Table 5). All the entries in Table 5 are close to 0 , showing that heterogeneous networks exhibited very small average changes in the vulnerability index $\xi$ when $E / I$ was varied. Thus, we conclude:

(3) for heterogeneous banking networks, global stabilities are affected very little by the amount of the total external asset $\mathscr{E}$ in the system.

Visual illustrations of (3) are shown in supplemental FIG. S4 and FIG. S5] for homogeneous and heterogeneous networks, respectively.

\subsection{Effect of network connectivity}

Although it is clear that connectivity properties of a banking network has a crucial effect on its stability, prior researchers have drawn mixed conclusions on this. For example, Allen and Gale [3] concluded that networks with less connectivity are more prone to contagion than networks with higher connectivity due to improved resilience of banking network topologies with higher connectivity via transfer of proportion of the losses in one bank's portfolio to more banks through interbank agreements. On the other hand, Babus [7] observed that, when the network connectivity is higher, liquidity can be redistributed in the system to make the risk of contagion lower, and Gai and Kapadia [33] observed that higher connectivity among banks leads to more contagion effect during a crisis. The mixed conclusions arise because links between banks have conceptually two conflicting effects on contagion, namely,

\footnotetext{
${ }^{17}$ For visual illustrations to this phenomena, see supplemental FIG. S1]S3 For example, in supplemental FIG. S1] when $\gamma$ is $45 \%$ and $\Phi$ is only $5 \%$ more than $\gamma$, the vulnerability index $\xi$ is approximately 0 for all the 9 combinations of parameters, but in supplemental FIG. S2 S3 all the 18 networks have a value of $\xi \geqslant 0.1$ even when $\gamma$ is $45 \%$ and the severity of the shock is only $5 \%$ more than $\gamma$.
} 
STABILITY OF FINANCIAL NETWORKS

Table 4. Residual instabilities of homogeneous versus heterogeneous networks under coordinated shocks. The percentages shown are the percentages of networks for which $\xi<0.05$ or $\xi<0.1$ or $\xi<0.2$. See also supplementary Tables S1 $\mathbf{S 9}$

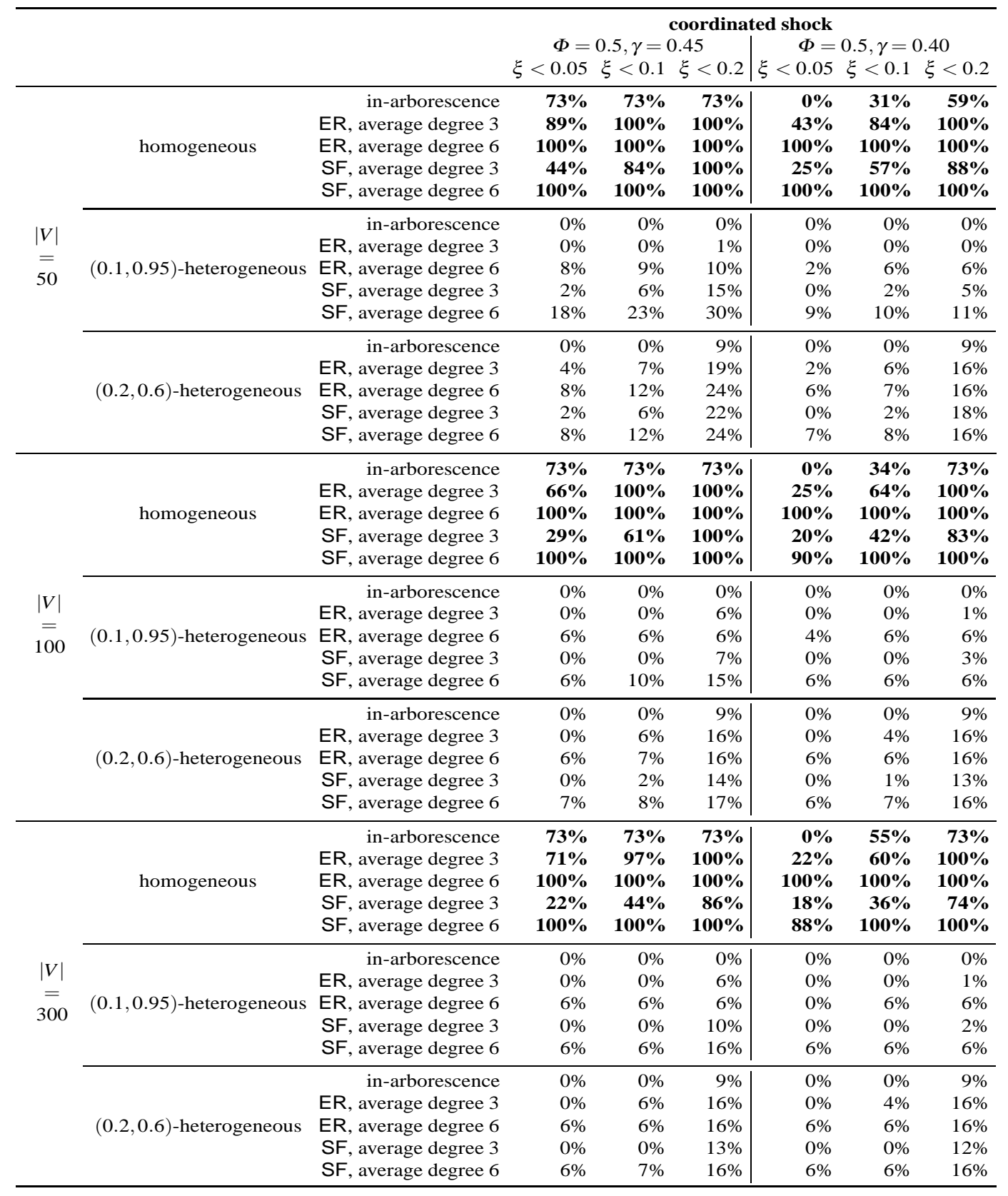


DASGUPTA AND KALIGOUNDER

Table 5. Absolute values of the largest change of the vulnerability index $\xi$ in the range $0.25 \leqslant \mathscr{E} / \mathscr{I} \leqslant 3.5$.

\begin{tabular}{rr|cc}
\hline average values of & $\max _{0.25 \leqslant \frac{\delta}{\zeta} \leqslant 3.5}\{\xi\}-\min _{0.25 \leqslant \frac{\delta}{\xi} \leqslant 3.5}\{\xi\} \mid$ \\
& \multicolumn{1}{c}{ coordinated shock } & idiosyncratic shock \\
\cline { 2 - 3 }$(0.1,0.95)$-heterogeneous in-arborescence & $\mathbf{0 . 0 1 7}$ & $\mathbf{0 . 0 4 5}$ \\
$(0.2,0.6)$-heterogeneous in-arborescence & $\mathbf{0 . 0 0 7}$ & $\mathbf{0 . 0 1 7}$ \\
$(0.1,0.95)$-heterogeneous ER, average degree 3 & $\mathbf{0 . 0 6 6}$ & $\mathbf{0 . 0 7 3}$ \\
$(0.2,0.6)$-heterogeneous ER, average degree 3 & $\mathbf{0 . 0 4 0}$ & $\mathbf{0 . 0 4 1}$ \\
$(0.1,0.95)$-heterogeneous ER, average degree 6 & $\mathbf{0 . 1 1 1}$ & $\mathbf{0 . 1 1 6}$ \\
$(0.2,0.6)$-heterogeneous ER, average degree 6 & $\mathbf{0 . 0 8 4}$ & $\mathbf{0 . 0 7 8}$ \\
$(0.1,0.95)$-heterogeneous SF, average degree 3 & $\mathbf{0 . 1 1 9}$ & $\mathbf{0 . 0 9 4}$ \\
$(0.2,0.6)$-heterogeneous SF, average degree 3 & $\mathbf{0 . 0 3 4}$ & $\mathbf{0 . 0 3 2}$ \\
$(0.1,0.95)$-heterogeneous SF, average degree 6 & $\mathbf{0 . 2 0 0}$ & $\mathbf{0 . 1 7 9}$ \\
$(0.2,0.6)$-heterogeneous SF, average degree 6 6 & $\mathbf{0 . 0 5 4}$ & $\mathbf{0 . 0 5 4}$ \\
\hline
\end{tabular}

- more interbank links increases the opportunity for spreading insolvencies to other banks,

- but, more interbank links also provide banks with co-insurance against fluctuating liquidity flows.

As our findings show, these two conflicting effects have different strengths in homogeneous versus highly heterogeneous networks, thus justifying the mixed conclusions of past researchers.

Table 6. Effect of connectivity on the stability for homogeneous networks under coordinated and idiosyncratic shocks. The percentage shown for a comparison of the type "network A versus network B" indicates the percentage of data points for which the stability of networks of type A was at least as much as that of networks of type B.

\begin{tabular}{|c|c|c|c|}
\hline \multicolumn{2}{|c|}{ ER average degree 3 versus ER average degree 6} & \multicolumn{2}{|c|}{ SF average degree 3 versus SF average degree 6} \\
\hline coordinated shock & idiosyncratic shock & coordinated shock & idiosyncratic shock \\
\hline $\mathbf{9 7 . 4 3 \%}$ & $97.05 \%$ & 98.89\% & $98.29 \%$ \\
\hline
\end{tabular}

Homogeneous networks Recall that in a homogeneous network all banks have the same external asset. Table 6 shows sparser homogeneous networks with lower average degrees to be more stable for same values of other parameters. Thus, we conclude:

(4) for homogeneous networks, higher connectivity leads to lower stability.

Heterogeneous networks In a heterogeneous network, there are banks that are "too big" in the sense that these banks have much larger external assets and inter-bank exposures compared to the remaining 
STABILITY OF FINANCIAL NETWORKS

Table 7. Effect of connectivity on the stability under coordinated and idiosyncratic shocks for (A) $(\alpha, \beta)$-heterogeneous ER and $\mathrm{SF}$ networks and $(\mathbf{B})(\alpha, \beta)$-heterogeneous in-arborescence versus $(\alpha, \beta)$-heterogeneous $\mathrm{SF}$ networks. The percentage shown for a comparison of the type "network A versus network B" indicates the percentage of data points for which the stability of networks of type A was at least as much as that of networks of type B.

\begin{tabular}{|c|c|c|c|c|c|c|c|}
\hline \multicolumn{2}{|c|}{$\begin{array}{c}(0,1,0.95) \text { ER average degree } 6 \\
\quad \text { versus } \\
(0,1,0.95) \text { ER average degree } 3\end{array}$} & \multicolumn{2}{|c|}{$\begin{array}{c}(0.2,0.6) \text { ER average degree } 6 \\
\quad \text { versus } \\
(0.2,0.6) \text { ER average degree } 3\end{array}$} & \multicolumn{2}{|c|}{$\mid \begin{array}{c}(0,1,0.95) \text { SF average degree } 6 \\
\quad \text { versus } \\
(0,1,0.95) \text { SF average degree } 3\end{array}$} & \multicolumn{2}{|c|}{$\begin{array}{c}(0.2,0.6) \text { SF average degree } 6 \\
\text { versus } \\
(0.2,0.6) \text { SF average degree } 3\end{array}$} \\
\hline $\begin{array}{l}\text { oordinated } \\
\text { shock }\end{array}$ & $\begin{array}{r}\text { idiosy } \\
\text { sh }\end{array}$ & $\begin{array}{c}\text { coordinated } \\
\text { shock }\end{array}$ & $\begin{array}{r}\text { idios } \\
\text { sl }\end{array}$ & $\begin{array}{c}\text { coordinated } \\
\text { shock }\end{array}$ & $\begin{array}{c}\text { idiosyncratic } \\
\text { shock }\end{array}$ & $\begin{array}{c}\text { coordinated } \\
\text { shock }\end{array}$ & $\begin{array}{l}\text { idiosyncratic } \\
\text { shock }\end{array}$ \\
\hline $89.3 \%$ & $82.39 \%$ & $68.12 \%$ & $61.46 \%$ & $85.51 \%$ & $73.81 \%$ & $69.29 \%$ & $73.07 \%$ \\
\hline
\end{tabular}

(A)

\begin{tabular}{|c|c|}
\hline $\begin{array}{c}(0,1,0.95) \text { SF average degree } 3 \text { and average degree } 6 \\
\quad \text { versus } \\
(0.1,0.95) \text {-heterogeneous in-arborescence (SF ave. degree } 1)\end{array}$ & $\begin{array}{c}(0.2,0.6) \mathrm{SF} \text { average degree } 3 \text { and average degree } 6 \\
\quad \text { versus } \\
(0.2,0.6) \text {-heterogeneous in-arborescence (SF ave. degree } 1)\end{array}$ \\
\hline idiosyncratic shock & idiosyncratic shock \\
\hline $81.86 \%$ & $51.07 \%$ \\
\hline
\end{tabular}

(B)

banks. Table 7 shows that for heterogeneous network models denser networks with higher average degree are more stable than the corresponding sparser networks for same values of other parameters, especially when the heterogeneity of the network is larger (i.e., when $\alpha=0.1, \beta=0.95$ ). Thus, we conclude:

\section{(5) for heterogeneous networks, higher connectivity leads to higher stability.}

\section{Formal intuition behind the conclusions in (4) and (5)}

Informally, conclusions (4) and (5) indicate that in homogeneous networks higher connectivity leads to more opportunity for spreading insolvencies to other banks whereas in heterogeneous networks higher connectivity provides banks with co-insurance against fluctuating liquidity flows through shared interbank assets. However, a precise formal treatment of mechanism that drives such conclusions is complicated due to several reasons such as the random nature of the networks, the randomness in asset distribution for heterogeneous networks and the non-linear nature of the insolvency propagation equation. Nevertheless, we provide the following, somewhat simplified, formal reasoning. We will use the following notations and conventions:

- $\operatorname{deg}_{\text {ave }}=\frac{|E|}{n}$ will denote the average degree of a graph $G$. It is assumed that $\operatorname{deg}_{\text {ave }}$ is a small positive integer constant independent of $n$ (e.g., in our simulation work, $\operatorname{deg}_{\text {ave }} \in\{1,3,6\}$ ).

- $\Delta x$ will denote a small change for the value $x$ of a variable.

\footnotetext{
${ }^{18}$ In standard algorithmic analysis terminologies, $f \approx g$ implies $\frac{f(r)}{g(r)}=1 \pm \mathrm{o}(1)$.
} 


\section{DASGUPTA AND KALIGOUNDER}

- For two functions $f(r)$ and $g(r)$ of a variable $r$, we will use the notation $f \approx g$ (respectively, $f \lesssim g$, $f \gtrsim g$ ) if $\lim _{r \rightarrow \infty} \frac{f(r)}{g(r)}=1$ (respectively, $\lim _{r \rightarrow \infty} \frac{f(r)}{g(r)} \leqslant 1, \lim _{r \rightarrow \infty} \frac{f(r)}{g(r)} \geqslant 1$ ).

- The standard phrase "with high probability" (or w.h.p. in short) refers to a probability $p(n)$ such that $\lim _{n \rightarrow \infty} p(n)=0$.

- If necessary, we will use the superscripts "homo" and "hetero" to denote the value of a quantity for homogeneous and heterogeneous networks, respectively.

Consider a node $v \in V_{\mathbf{x}}$ with $\operatorname{deg}^{\operatorname{in}}(v)>1$ and suppose that $v$ fails due to the initial shock at $t=0$. By Equation (5.1), for every edge $(u, v) \in E$, the amount of shock $u$ receives from $v$ is given by $\mathbb{B}=$ $\min \left\{\mathbb{A}, c_{1}\right\}$ with

$$
\begin{aligned}
\mathbb{A} & =\frac{\Phi\left(c_{1} \operatorname{deg}^{\text {in }}(v)-c_{1} \operatorname{deg}^{\text {out }}(v)+c_{2} \mathscr{E}\right)-\gamma\left(c_{1} \operatorname{deg}^{\text {in }}(v)+c_{2} \mathscr{E}\right)}{\operatorname{deg}^{\text {in }}(v)} \\
& =c_{1}(\Phi-\gamma)+c_{2}(\Phi-\gamma) \frac{\mathscr{E}}{\operatorname{deg}^{\text {in }}(v)}-c_{1} \Phi \frac{\operatorname{deg}^{\text {out }}(v)}{\operatorname{deg}^{\text {in }}(v)}
\end{aligned}
$$

for some appropriate positive quantities $c_{1}$ and $c_{2}$ that may be estimated as follows:

- If $G$ is homogeneous then $c_{1}^{\text {homo }}=\frac{\mathscr{I}}{n \operatorname{deg}_{\text {ave }}}=1$ and $c_{2}^{\text {homo }}=\frac{1}{n}$.

- If $G$ is $(\alpha, \beta)$-heterogeneous then $c_{1}^{\text {hetero }}$ and $c_{2}^{\text {hetero }}$ are random variables independent of deg ave. Using the notations in Definition 6.1 the expected value of $c_{2}^{\text {hetero }}$ may be estimated as follows:

$$
\mathbb{E}\left[c_{2}^{\text {hetero }}\right]=\frac{\operatorname{Pr}[v \in \widetilde{V}] \frac{\beta \mathscr{E}}{\alpha n}+\operatorname{Pr}[v \notin \widetilde{V}] \frac{(1-\beta) \mathscr{E}}{(1-\alpha) n}}{\mathscr{E}}=\frac{\alpha \frac{\beta \mathscr{E}}{\alpha n}+(1-\alpha) \frac{(1-\beta) \mathscr{E}}{(1-\alpha) n}}{\mathscr{E}}=\frac{1}{n}
$$

The expected value of $c_{1}^{\text {hetero }}$ depend on the nature (SF or ER) of the random network; its estimation is therefore deferred until later.

Our goal is to provide evidence for a claim of the following nature for either random SF or random ER networks:

For many realistic network parameter combinations, $\mathrm{w} . \mathrm{h} \cdot \mathrm{p}$. increasing connectivity from $d$ to $d+\Delta d$ decreases the expected amount of shock transmitted by a failed node $v$ to $u$ in homogeneous networks (causing improved stability) but increases the expected amount of shock transmitted by a failed node $v$ to $u$ in heterogeneous networks (leading to worse stability).

The case of random SF networks If $G$ is a directed SF network, then the discrete probability density function for the degree of any node $v$ in $G$ is given by:

$$
\forall k \in\{1,2, \ldots, n-1\}: \operatorname{Pr}\left[\operatorname{deg}^{\text {in }}(v)=k\right]=\operatorname{Pr}\left[\operatorname{deg}^{\text {out }}(v)=k\right]=C k^{-\mu}
$$

where $\mu>2$ is the constant for the exponent of the distribution and $C>0$ is a constant such that $\mathbb{E}\left[\operatorname{deg}^{\text {in }}(v)\right]=\mathbb{E}\left[\operatorname{deg}^{\text {out }}(v)\right]=\operatorname{deg}_{\text {ave. }}$ For example, for our random in-arborescence networks, the results in [8] imply $\mu=3$. To simplify exposure, in the following we assume that $\mu=3$, though 


\section{STABILITY OF FINANCIAL NETWORKS}

the analysis can be extended in a straightforward manner for any other $\mu>2 . \zeta(s)=\sum_{x=1}^{\infty} x^{-s}$ is the well-known Riemann zeta function; it is well known that $\zeta(s) \approx \sum_{x=1}^{n-1} x^{-s}$ for any $s>2$ and for all large $n$ (see [44, page 74-75]) and $\zeta(s)<\frac{2^{s-1}}{2^{s-1}-1}$ for any $s>1$ (see [44, page 489]). In particular, it is known that $\zeta(2)=\frac{\pi^{2}}{6}, \zeta(3)=1.2020569 \cdots$ and $\zeta(4)=\frac{\pi^{4}}{90}$. Note that

$$
\mathbb{E}\left[\operatorname{deg}^{\text {in }}(v)\right]=\operatorname{deg}_{\text {ave }} \equiv \sum_{k=1}^{n-1} k\left(C k^{-3}\right)=\operatorname{deg}_{\text {ave }} \Rightarrow C \approx \frac{\operatorname{deg}_{\text {ave }}}{\zeta(2)}=\frac{6 \operatorname{deg}_{\text {ave }}}{\pi^{2}}
$$

LEMmA 7.2 (see Section $\mathbb{B}$ of the appendix for a proof) $\mathbb{E}\left[\frac{1}{\operatorname{deg}^{\text {in }}(v)} \mid \operatorname{deg}^{\text {in }}(v)>0\right] \approx \frac{\pi^{2}}{15} \operatorname{deg}_{\text {ave }}$ and $\operatorname{Var}\left[\operatorname{deg}^{\text {in }}(v)\right] \approx \frac{6 \operatorname{deg}_{\text {ave }}}{\pi^{2}} \ln n$.

We now provide an estimation of $c_{1}^{\text {hetero }}$ using the notations in Definition 6.1.

LEMma 7.3 (see Section $\$ of the appendix for a proof) w.h.p. $\quad 1+\alpha-\beta-\frac{\alpha \beta}{2} \leqslant \mathbb{E}\left[c_{1}^{\text {hetero }}\right] \leqslant$ $\frac{1+\alpha \beta-\alpha^{2}-\beta-\alpha^{2} \beta}{1-2 \alpha}$.

Due to the above lemma, we may assume that

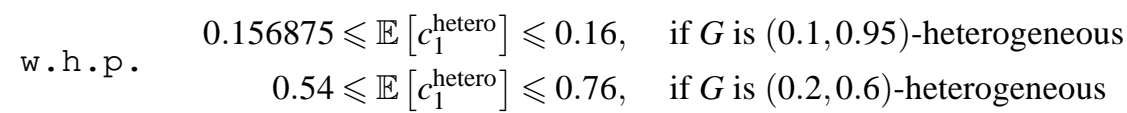

We will investigate the sensitivity of the amount of shock $\mathbb{A}$ transmitted from $v$ to $u$ as the average degree $\operatorname{deg}_{\text {ave }}$ is changed while keeping all other parameters the same. For notational convenience, the parameters are normalized such that $\mathscr{I}=n \mathrm{deg}_{\text {ave }}$ at the initial value $d$ of deg ave. As deg ave is increased from $d$ to $d+\Delta d, \mathscr{I}$ is still kept the same. Thus,

$$
\left.\mathscr{I}\right|_{\operatorname{deg}_{\operatorname{ave}}^{\text {at }=d+\Delta d}}=\left.\left.\mathscr{I}\right|_{\operatorname{deg}_{\text {ave }=d}^{\text {at }}=d} \Rightarrow c_{1}^{\text {homo }}\right|_{\operatorname{deg}_{\text {ave }=d+\Delta d}^{\text {at }}=d}=\frac{\left.\mathscr{I}\right|_{\operatorname{deg}_{\text {ave }}^{\text {at }}=d+\Delta d}}{n(d+\Delta d)}=\frac{n d}{n(d+\Delta d)}=\frac{d}{d+\Delta d}
$$

Equation (7.1) gives the following for homogeneous and heterogeneous networks:

- If $G$ is a homogeneous network then

$$
\begin{aligned}
& \mathbb{E}\left[\mathbb{A}^{\text {homo }} \mid \operatorname{deg}_{\text {ave }}=d\right] \\
\approx & (\Phi-\gamma)+\frac{\mathscr{E}(\Phi-\gamma)}{n} \mathbb{E}\left[\frac{1}{\operatorname{deg}^{\operatorname{in}(v)}} \mid \operatorname{deg}^{\text {in }}(v)>0\right]-\Phi \mathbb{E}\left[\operatorname{deg}^{\text {out }}(v)\right] \mathbb{E}\left[\frac{1}{\operatorname{deg}^{\text {in }}(v)} \mid \operatorname{deg}^{\text {in }}(v)>0\right] \\
= & (\Phi-\gamma)+\frac{\pi^{2} \mathscr{E}(\Phi-\gamma)}{15 n} d-\frac{\pi^{2} \Phi}{15} d^{2} \\
& \mathbb{E}\left[\mathbb{A}^{\text {homo }} \mid \operatorname{deg}_{\text {ave }}=d+\Delta d\right] \\
\approx & \frac{d}{d+\Delta d}(\Phi-\gamma)+\frac{\mathscr{E}(\Phi-\gamma)}{n} \mathbb{E}\left[\frac{1}{\operatorname{deg}^{\text {in }(v)}} \mid \operatorname{deg}^{\operatorname{in}(v)>0]-\frac{d}{d+\Delta d}} \Phi \mathbb{E}\left[\operatorname{deg}^{\text {out }}(v)\right] \mathbb{E}\left[\frac{1}{\operatorname{deg}^{\text {in }}(v)} \mid \operatorname{deg}^{\operatorname{in}(v)>0}\right]\right.
\end{aligned}
$$


DASGUPTA AND KALIGOUNDER

$$
\begin{aligned}
= & \frac{d}{d+\Delta d}(\Phi-\gamma)+\frac{\pi^{2} \mathscr{E}(\Phi-\gamma)}{15 n}(d+\Delta d)-\frac{d}{d+\Delta d} \frac{\pi^{2} \Phi}{15}(d+\Delta d)^{2} \\
& \Delta \mathbb{E}\left[\mathbb{A}^{\text {homo }}\right] \\
= & \mathbb{E}\left[\mathbb{A}^{\text {homo }} \mid \operatorname{deg}_{\text {ave }}=d+\Delta d\right]-\mathbb{E}\left[\mathbb{A}^{\text {homo }} \mid \operatorname{deg}_{\text {ave }}=d\right] \\
\approx & -\frac{\Delta d}{d+\Delta d}(\Phi-\gamma)+\frac{\pi^{2} \mathscr{E}(\Phi-\gamma)}{15 n} \Delta d-\frac{\pi^{2} \Phi}{15} d \Delta d
\end{aligned}
$$

- If $G$ is a heterogeneous network then

$$
\begin{aligned}
\mathbb{E}\left[\mathbb{A}^{\text {hetero }}\right]=(\Phi-\gamma) \mathbb{E}\left[c_{1}^{\text {hetero }}\right]+ & \frac{\mathscr{E}(\Phi-\gamma)}{n} \mathbb{E}\left[\frac{1}{\operatorname{deg}^{\text {in }}(v)} \mid \operatorname{deg}^{\text {in }}(v)>0\right] \\
& -\Phi \mathbb{E}\left[c_{1}^{\text {hetero }}\right] \mathbb{E}\left[\operatorname{deg}^{\text {out }}(v)\right] \mathbb{E}\left[\frac{1}{\operatorname{deg}^{\text {in }}(v)} \mid \operatorname{deg}^{\text {in }}(v)>0\right]
\end{aligned}
$$

which provides the following bounds:

$$
\begin{aligned}
\mathbb{E}\left[\mathbb{A}^{\text {hetero }} \mid \operatorname{deg}_{\text {ave }}=d\right] & \approx(\Phi-\gamma) \mathbb{E}\left[c_{1}^{\text {hetero }}\right]+\frac{\pi^{2} \mathscr{E}(\Phi-\gamma)}{15 n} d-\frac{\pi^{2} \Phi}{15} d^{2} \mathbb{E}\left[c_{1}^{\text {hetero }}\right] \\
\mathbb{E}\left[\mathbb{A}^{\text {hetero }} \mid \operatorname{deg}_{\text {ave }}=d+\Delta d\right] & \approx(\Phi-\gamma) \mathbb{E}\left[c_{1}^{\text {hetero }}\right]+\frac{\pi^{2} \mathscr{E}(\Phi-\gamma)}{15 n}(d+\Delta d)-\frac{\pi^{2} \Phi}{15} d^{2} \mathbb{E}\left[c_{1}^{\text {hetero }}\right] \\
\Delta \mathbb{E}\left[\mathbb{A}^{\text {hetero }}\right] & =\mathbb{E}\left[\mathbb{A}^{\text {hetero }} \mid \operatorname{deg}_{\text {ave }}=d+\Delta d\right]-\mathbb{E}\left[\mathbb{A}^{\text {hetero }} \mid \operatorname{deg}_{\text {ave }}=d\right] \\
& \approx \frac{\pi^{2} \mathscr{E}(\Phi-\gamma)}{15 n} \Delta d-\frac{\pi^{2} \Phi}{15} d \Delta d \mathbb{E}\left[c_{1}^{\text {hetero }}\right]
\end{aligned}
$$

Now note that

$$
\begin{array}{r}
\Delta \mathbb{E}\left[\mathbb{A}^{\text {homo }}\right] \lesssim 0 \Rightarrow \frac{\Phi-\gamma}{d+\Delta d}+\frac{\pi^{2} \Phi d}{15}>\frac{\pi^{2} \mathscr{E}(\Phi-\gamma)}{15 n} \\
\equiv\left(\frac{1}{d+\Delta d}+\frac{\pi^{2} d}{15}-\frac{\pi^{2} \mathscr{E}}{15 n}\right) \Phi>\left(\frac{1}{d+\Delta d}-\frac{\pi^{2} \mathscr{E}}{15 n}\right) \gamma \\
\Delta \mathbb{E}\left[\mathbb{A}^{\text {hetero }}\right] \gtrsim 0 \Rightarrow \frac{\mathscr{E}(\Phi-\gamma)}{n}>\Phi d \mathbb{E}\left[c_{1}^{\text {hetero }}\right] \equiv\left(\frac{\mathscr{E}}{n}-d \mathbb{E}\left[c_{1}^{\text {hetero }}\right]\right) \Phi>\left(\frac{\mathscr{E}}{n}\right) \gamma \\
\Rightarrow \text { w.h.p. } \quad\left(\frac{\mathscr{E}}{n}-d \frac{1+\alpha \beta-\alpha^{2}-\beta-\alpha^{2} \beta}{1-2 \alpha}\right) \Phi>\left(\frac{\mathscr{E}}{n}\right) \gamma
\end{array}
$$

It is easy to verify that constraints (7.2) and (7.3) are satisfied for many natural combinations of parameters. In fact, the constraints $(\mathbf{7 . 2})$ and $(7.3)$ are almost always satisfied when $\mathscr{E}$ grows moderately linearly 
with $n$. To see this informally, note that since $\alpha \ll \beta$ and $\alpha$ is small, $\frac{1+\alpha \beta-\alpha^{2}-\beta-\alpha^{2} \beta}{1-2 \alpha} \approx 1-\beta$ and thus (7.3) is approximately

$$
\left(\frac{\mathscr{E}}{n}-d(1-\beta)\right) \Phi>\left(\frac{\mathscr{E}}{n}\right) \gamma
$$

Now suppose that $\mathscr{E}<d(1-\beta) n$. Then, (7.2) and (7.3) $)$ are always satisfied since $\Phi>\gamma$. For a numerical example, suppose that $G$ is a $(0.1,0.95)$-heterogeneous network (i.e., $\alpha=0.1$ and $\beta=0.95$ ), $d=3, \Delta d=1, \gamma=0.2$ and $\Phi=0.4$. Then constraints (7.2) and (7.3) reduce to:

$$
0.5+0.4 \pi^{2}-\frac{2 \pi^{2} \mathscr{E}}{15 n}>0.25-\frac{\pi^{2} \mathscr{E}}{15 n} \quad \text { and } \quad \frac{2 \mathscr{E}}{n}-0.94125>\frac{\mathscr{E}}{n}
$$

and these constraints can be satisfied when $\mathscr{E}$ grows moderately linearly with $n$, i.e., when $0.94125 n<$ $\mathscr{E}<6.38 n$.

The case of random ER networks In a random ER network, the probability of having a particular edge is given by the following set of independent Bernoulli trials:

$$
\forall u, v \in V \text { with } u \neq v: \operatorname{Pr}[(u, v) \in E]=p=\frac{\operatorname{deg}_{\text {ave }}}{n-1}
$$

Thus, for every $k \in\{0,1,2, \ldots, n-1\}$ :

$$
\begin{aligned}
\operatorname{Pr}\left[\operatorname{deg}^{\text {in }}(v)=k\right] & =\operatorname{Pr}\left[\operatorname{deg}^{\text {out }}(v)=k\right]=\left(\begin{array}{c}
n-1 \\
k
\end{array}\right) p^{k}(1-p)^{(n-1)-k} \\
\mathbb{E}\left[\operatorname{deg}^{\text {in }}\right] & =\mathbb{E}\left[\operatorname{deg}^{\text {out }}\right]=p(n-1)=\operatorname{deg}_{\text {ave }} \\
\operatorname{Var}\left[\operatorname{deg}^{\text {in }}(v)\right] & =\operatorname{Var}\left[\operatorname{deg}^{\text {out }}(v)\right]=p(1-p)(n-1)=\left(1-\frac{\operatorname{deg}_{\text {ave }}}{n-1}\right) \operatorname{deg}_{\text {ave }}
\end{aligned}
$$

Since deg $\operatorname{ave}=p(n-1)$ is a constant, one can approximate the above binomial distribution by a Poisson's distribution [57, page 72] to obtain

$$
\operatorname{Pr}\left[\operatorname{deg}^{\text {in }}(v)=k\right]=\operatorname{Pr}\left[\operatorname{deg}^{\text {out }}(v)=k\right] \approx \mathrm{e}^{-\operatorname{deg}_{\text {ave }}} \frac{\left(\operatorname{deg}_{\text {ave }}\right)^{k}}{k !}
$$

LEMMA 7.4 (see SectionDof the appendix for a proof) $\left|\mathbb{E}\left[\frac{1}{\operatorname{deg}^{\text {in }}(v)}\right]-\sum_{k=1}^{\left\lfloor 3 \operatorname{deg}_{\text {ave }}+10\right\rfloor}(1 / k) \mathrm{e}^{-\operatorname{deg}_{\text {ave }}} \frac{\left(\operatorname{deg}_{\text {ave }}\right)^{k}}{k !}\right| \lesssim$ $10^{-10}$ and $\frac{\partial}{\partial d} \mathbb{E}\left[\frac{1}{\operatorname{deg}(v)} \mid \operatorname{deg}_{\mathrm{ave}}=d\right] \approx \frac{1-\mathrm{e}^{-d}}{d}$.

For notational convenience, let $\Upsilon(x)=\sum_{k=1}^{\lfloor 3 x+10\rfloor} \frac{1}{k} \mathrm{e}^{-x} \frac{x^{k}}{k !}$. Since $10^{-10}$ is an extremely small number for our purposes, we will just use $\mathbb{E}\left[\frac{1}{\operatorname{deg}^{\mathrm{in}}(v)}\right] \approx \Upsilon\left(\operatorname{deg}_{\text {ave }}\right)$ in the sequel. Values of $\Upsilon(x)$ and $\frac{\partial \Upsilon(x)}{\partial x}=\frac{1-\mathrm{e}^{-x}}{x}$ for a few small integral values of $x$ are shown in Table 8 It is easy to see that $\lim _{x \rightarrow \infty} \Upsilon(x)=0$ and, for large $x, \frac{\partial \Upsilon(x)}{\partial x} \approx 1 / x$.

As before, we first provide an estimation of $c_{1}^{\text {hetero }}$ using the notations in Definition 6.1 . 
DASGUPTA AND KALIGOUNDER

Table 8 . Values of $\Upsilon(x)$ and $\frac{\partial \Upsilon(x)}{\partial x}$ for a few small integral values of $x$ using straightforward calculations.

\begin{tabular}{l|ccccccccccc}
\hline & & & \multicolumn{1}{c}{$x=$} \\
$r(x) \approx$ & 2 & 3 & 4 & 5 & 6 & 7 & 8 & 9 & 10 & 11 & 12 \\
\hline & 0.499 & 0.411 & 0.324 & 0.256 & 0.207 & 0.172 & 0.147 & 0.128 & 0.113 & 0.101 & 0.091 \\
\hline$\frac{\partial r(x)}{x} \approx$ & 0.432 & 0.284 & 0.245 & 0.256 & 0.199 & 0.166 & 0.143 & 0.125 & 0.111 & 0.100 & 0.090 \\
\hline
\end{tabular}

LEMmA 7.5 (see Section $\mathbb{E}$ of the appendix for a proof) w.h.p. $1+\alpha-\beta-\frac{\alpha \beta}{2} \leqslant \mathbb{E}\left[c_{1}^{\text {hetero }}\right] \leqslant$ $\frac{1+\alpha \beta-\alpha^{2}-\beta-\alpha^{2} \beta}{1-2 \alpha}$.

Using the above result, the following bounds hold:

- If $G$ is a homogeneous network then

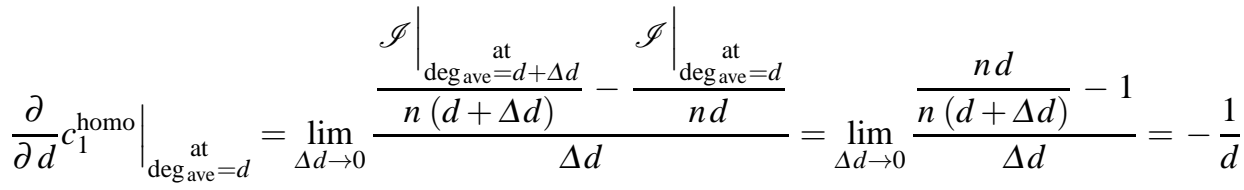

$$
\begin{aligned}
& \mathbb{E}\left[\mathbb{A}^{\text {homo }} \mid \operatorname{deg}_{\text {ave }}=d\right] \\
& =c_{1}^{\text {homo }}(\Phi-\gamma)+\frac{\mathscr{E}(\Phi-\gamma)}{n} \mathbb{E}\left[\frac{1}{\operatorname{deg}^{\operatorname{in}(v)}} \mid \operatorname{deg}^{\text {in }}(v)>0\right]-c_{1}^{\text {homo }} \Phi \mathbb{E}\left[\operatorname{deg}^{\text {out }}(v)\right] \mathbb{E}\left[\frac{1}{\operatorname{deg}^{\operatorname{in}(v)}} \mid \operatorname{deg}^{\operatorname{in}}(v)>0\right] \\
& \approx c_{1}^{\text {homo }}(\Phi-\gamma)+\frac{\mathscr{E}(\Phi-\gamma)}{n} \Upsilon(d)-c_{1}^{\text {homo }} \Phi d \Upsilon(d) \\
& \frac{\partial}{\partial d} \mathbb{E}\left[\mathbb{A}^{\text {homo }} \mid \operatorname{deg}_{\text {ave }}=d\right] \\
& \left.\approx(\Phi-\gamma) \frac{\partial}{\partial d} c_{1}^{\text {homo }}\right|_{\operatorname{deg}_{\text {ave }=d}^{\text {at }}}+\frac{\mathscr{E}(\Phi-\gamma)}{n} \frac{\partial}{\partial d} \Upsilon(d)-\Phi \frac{\partial}{\partial d}\left(\left.c_{1}^{\text {homo }}\right|_{\substack{\text { deg ave }=d \\
\text { at }}} d \Upsilon(d)\right) \\
& =-\frac{\Phi-\gamma}{d}+\left(\frac{\mathscr{E}(\Phi-\gamma)}{n}\right)\left(\frac{1-\mathrm{e}^{-d}}{d}\right)-\Phi\left(-\Upsilon(d)+\Upsilon(d)+1-\mathrm{e}^{-d}\right) \quad \begin{array}{l}
\text { using product rule } \\
\text { of derivatives }
\end{array} \\
& =-\frac{\Phi-\gamma}{d}+\left(\frac{\mathscr{E}(\Phi-\gamma)}{n}\right)\left(\frac{1-\mathrm{e}^{-d}}{d}\right)-\Phi\left(1-\mathrm{e}^{-d}\right)
\end{aligned}
$$


- If $G$ is a heterogeneous network then

$$
\begin{aligned}
\mathbb{E}\left[\mathbb{A}^{\text {hetero }}\right]=(\Phi-\gamma) \mathbb{E}\left[c_{1}^{\text {hetero }}\right]+ & \frac{\mathscr{E}(\Phi-\gamma)}{n} \mathbb{E}\left[\frac{1}{\operatorname{deg}^{\text {in }}(v)} \mid \operatorname{deg}^{\text {in }}(v)>0\right] \\
& -\Phi \mathbb{E}\left[c_{1}^{\text {hetero }}\right] \mathbb{E}\left[\operatorname{deg}^{\text {out }}(v)\right] \mathbb{E}\left[\frac{1}{\operatorname{deg}^{\text {in }}(v)} \mid \operatorname{deg}^{\text {in }}(v)>0\right]
\end{aligned}
$$

which provides the following bounds w.h.p.:

$$
\begin{aligned}
& \mathbb{E}\left[\mathbb{A}^{\text {hetero }} \mid \operatorname{deg}_{\text {ave }}=d\right] \approx(\Phi-\gamma) \mathbb{E}\left[c_{1}^{\text {hetero }}\right]+\frac{\mathscr{E}(\Phi-\gamma)}{n} \Upsilon(d)-\Phi d \Upsilon(d) \mathbb{E}\left[c_{1}^{\text {hetero }}\right] \\
& \frac{\partial}{\partial d} \mathbb{E}\left[\mathbb{A}^{\text {hetero }} \mid \operatorname{deg}_{\text {ave }}=d\right] \\
& \gtrsim \frac{\mathscr{E}(\Phi-\gamma)}{n} \frac{\partial}{\partial d} \Upsilon(d)-\Phi\left(\frac{1+\alpha \beta-\alpha^{2}-\beta-\alpha^{2} \beta}{1-2 \alpha}\right) \frac{\partial}{\partial d}(d \Upsilon(d)) \\
& =\left(\frac{\mathscr{E}(\Phi-\gamma)}{n}\right)\left(\frac{1-\mathrm{e}^{-d}}{d}\right)-\Phi\left(\frac{1+\alpha \beta-\alpha^{2}-\beta-\alpha^{2} \beta}{1-2 \alpha}\right)\left(1-\mathrm{e}^{-d}+\Upsilon(d)\right) \begin{array}{l}
\text { using product } \\
\text { rule of } \\
\text { derivatives }
\end{array}
\end{aligned}
$$

Now note the following:

$$
\begin{aligned}
& \frac{\partial}{\partial d} \mathbb{E}\left[\mathbb{A}^{\text {homo }} \mid \operatorname{deg}_{\text {ave }}=d\right] \geqq 0 \\
& \Rightarrow \frac{\Phi-\gamma}{d}+\Phi\left(1-\mathrm{e}^{-d}\right)>\left(\frac{\mathscr{E}(\Phi-\gamma)}{n}\right)\left(\frac{1-\mathrm{e}^{-d}}{d}\right) \\
& \equiv\left(\frac{1}{d}+1-\mathrm{e}^{-d}-\frac{\mathscr{E}}{n}\left(\frac{1-\mathrm{e}^{-d}}{d}\right)\right) \Phi>\left(\frac{1}{d}-\frac{\mathscr{E}}{n}\left(\frac{1-\mathrm{e}^{-d}}{d}\right)\right) \gamma \\
& \frac{\partial}{\partial d} \mathbb{E}\left[\mathbb{A}^{\text {hetero }} \mid \operatorname{deg}_{\text {ave }}=d\right] \gtrsim 0 \\
& \Rightarrow\left(\frac{\mathscr{E}(\Phi-\gamma)}{n}\right)\left(\frac{1-\mathrm{e}^{-d}}{d}\right)>\Phi\left(\frac{1+\alpha \beta-\alpha^{2}-\beta-\alpha^{2} \beta}{1-2 \alpha}\right)\left(1-\mathrm{e}^{-d}+\Upsilon(d)\right) \\
& \equiv\left(\frac{\mathscr{E}}{n}\left(\frac{1-\mathrm{e}^{-d}}{d}\right)-\left(\frac{1+\alpha \beta-\alpha^{2}-\beta-\alpha^{2} \beta}{1-2 \alpha}\right)\left(1-\mathrm{e}^{-d}+\Upsilon(d)\right)\right) \Phi>\frac{\mathscr{E}}{n}\left(\frac{1-\mathrm{e}^{-d}}{d}\right) \gamma
\end{aligned}
$$

It is easy to verify that constraints (7.4) and (7.5) are satisfied for many natural combinations of parameters. For example, the constraints (7.4) and (7.5) are almost always satisfied if $d$ is sufficiently large. To see this informally, note that if $d$ is large then $\frac{1}{d}, \mathrm{e}^{-d}, \Upsilon(d) \approx 0$. Moreover, since $\alpha \ll \beta$ and $\alpha$ is small, $\frac{1+\alpha \beta-\alpha^{2}-\beta-\alpha^{2} \beta}{1-2 \alpha} \approx 1-\beta$ and then constraints (7.4) and (7.5) reduce to

$$
\left(1-\frac{\mathscr{E}}{n d}\right) \Phi \gtrsim-\frac{\mathscr{E}}{n d} \gamma \equiv \Phi \lesssim \frac{\frac{\mathscr{E}}{n d}}{\frac{\mathscr{E}}{n d}-1} \gamma
$$


DASGUPTA AND KALIGOUNDER

Table 9. Comparisons of strengths of coordinated versus idiosyncratic shocks. The percentages indicate the percentage of total number of data points (combinations of parameters $\Phi, \gamma, \mathscr{E}$ and $\mathscr{K}$ ) for that network type that resulted in $\xi_{c} \geqslant \xi_{r}$, where $\xi_{c}$ and $\xi_{r}$ are the vulnerability indices under coordinated and idiosyncratic shocks, respectively.

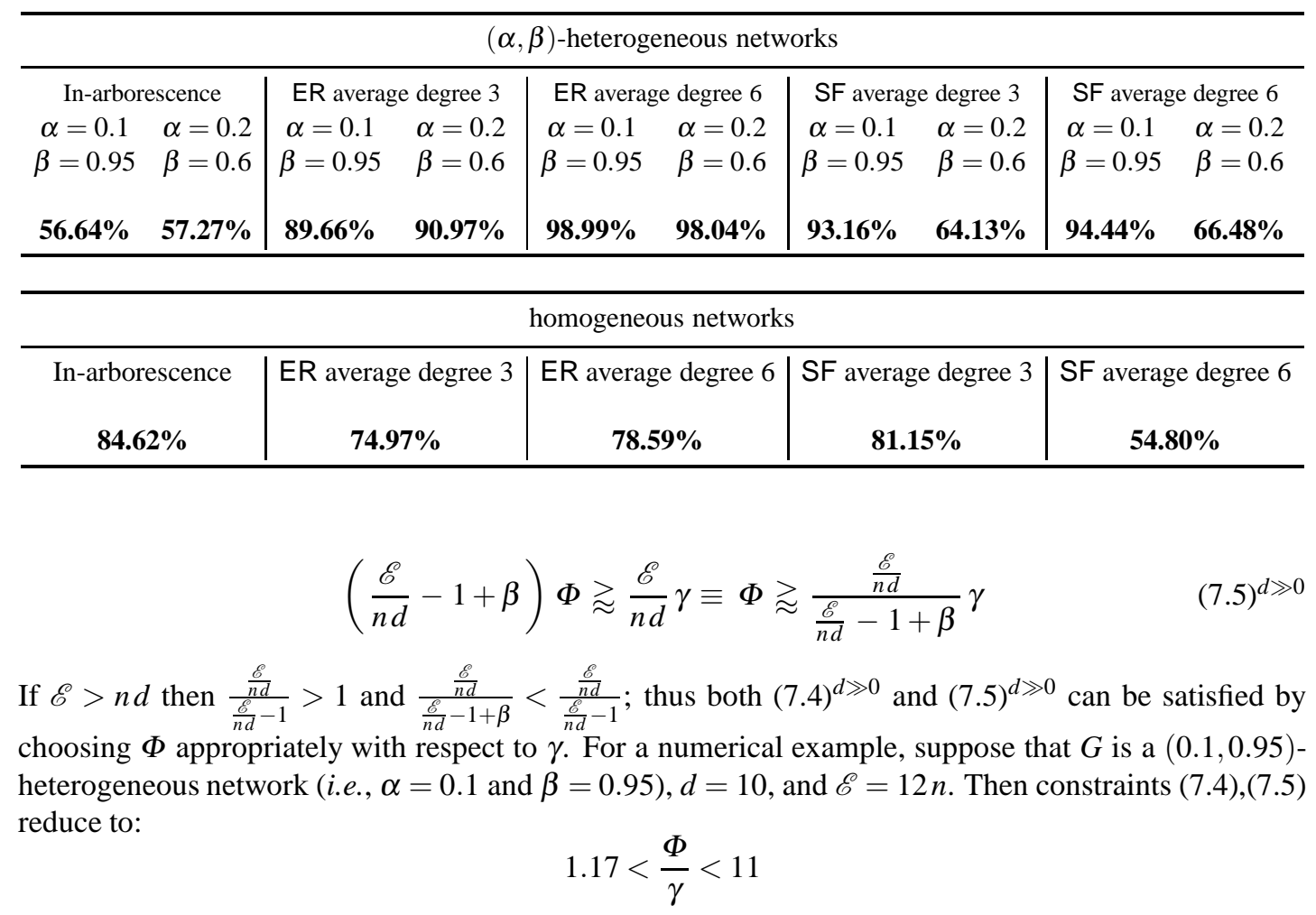

which corresponds to most settings of $\Phi$ and $\gamma$ used in our simulation.

\subsection{Random versus correlated initial failures}

For most parameter combinations, our results, tabulated in Table 9 , show that coordinated shocks, which are a type of correlated shocks, resulted in insolvencies of higher number of nodes as opposed to idiosyncratic shocks for the same network with the same parameters, often by a factor of two or more. For example, Table 9 shows that for $(0.1,0.95)$-heterogeneous ER networks of average degree 6 the vulnerability index under coordinated shocks is at least as much as the vulnerability index under idiosyncratic shocks $98.99 \%$ of the time. Thus, we conclude:

(6) correlated shocking mechanisms are more appropriate to measure the worst-case stability compared to idiosyncratic shocking mechanisms.

For visual illustrations of (6), see supplemental FIG. S6 -S11.

\subsection{Phase transition properties of stability}

Phase transitions are quite common when one studies various topological properties of graphs [10]. The stability measure exhibits several sharp phase transitions for various banking networks and com- 


\section{STABILITY OF FINANCIAL NETWORKS}

binations of parameters; see supplemental FIG. $\mathbf{S 1}-\mathbf{S} 4$ for visual illustrations. We discuss two such interesting phase transitions in the following, with an intuitive theoretical explanation for one of them.

7.5.1 Dense homogeneous networks Based on the behavior of $\xi$ with respect to $(\Phi-\gamma)$, we observe that, for smaller value of $\mathscr{K}$ and for denser ER and SF networks under either coordinated or idiosyncratic shocks, there is often a sharp decrease of stability when $\gamma$ was decreased beyond a particular threshold value. For example, with $\Phi=0.5$ and $\mathscr{K}=0.1,100$-node dense (average degree 6) SF and ER homogeneous networks exhibited more than ninefold increase in $\xi$ around $\gamma=0.15$ and $\gamma=0.1$, respectively; see supplemental FIG. S1 for visual illustrations.

To investigate the global extent of such a sharp decrease at a threshold value of $\gamma$ in the range $[0.05,0.2]$, we computed, for each of the five homogeneous network types under coordinated shocks and for each values of the parameters $|V|, \Phi, \frac{\mathscr{E}}{\mathscr{I}}$ and $\mathscr{K}$, , the ratio

$$
\Lambda\left(n, \Phi, \frac{\mathscr{E}}{\mathscr{I}}, \mathscr{K}\right)=\frac{\max _{0.05 \leqslant \gamma \leqslant 0.2}\{\xi\}-\min _{0.05 \leqslant \gamma \leqslant 0.2}\{\xi\}}{\max _{\text {entire range of } \gamma}\{\xi\}-\min _{\text {entire range of } \gamma}\{\xi\}}
$$

that provides the maximum percentage of the total change of the vulnerability index that occurred within this narrow range of $\gamma$. The values of $\Lambda\left(n, \Phi, \frac{\mathscr{E}}{\mathscr{J}}, \mathscr{K}\right)$ for the dense ER and SF homogeneous networks under coordinated shocks are shown in Table 10 for $\Phi=0.5,0.8$ and $\mathscr{K}=0.1,0.2,0.3,0.4,0.5$ (the behaviour of $\xi$ is similar for other intermediate values of $\Phi$ ). If the growth of $\xi$ with respect to $\gamma$ was uniform or near uniform over the entire range of $\gamma, \Lambda$ would be approximately $\lambda=\frac{0.2-0.05}{0.45-0.05}=0.375$; thus, any value of $\Lambda$ significantly higher than $\lambda$ indicates a sharp transition within the above-mentioned range of values of $\gamma$. As Table 10 shows, a significant majority of the entries for $\Phi \leqslant 0.8$ and $\kappa \leqslant 0.2$ are $2 \lambda$ or more.

7.5.2 Homogeneous in-arborescence networks Homogeneous in-arborescence networks under coordinated shocks exhibited a sharp increase in stability as the ratio $\frac{\mathscr{E}}{\mathscr{I}}$ of the total external asset to the total interbank exposure the system is increased beyond a particular threshold provided the equity to asset ratio $\gamma$ was approximately $\Phi / 2$. For example, for a 50-node homogeneous in-arborescence network under coordinated shock, $\xi$ exhibited a sharp decrease from 0.76 to 0.18 for $\frac{\mathscr{E}}{\mathscr{I}} \in[0.75,1], \mathscr{K}=0.1$, $\Phi=0.5$ and $\gamma=0.25=\Phi / 2$; see supplemental FIG. S4 for a visual illustration.

To investigate the global extent of such a sharp decrease of $\xi$ around a threshold value of $\frac{\mathscr{E}}{\mathscr{I}}$ in the range $[0.5,1]$ with $\gamma \approx \Phi / 2$, we computed, for each type of shocking mechanism, and for each values of the parameters $n, \Phi, \gamma \approx \Phi / 2$, and $\kappa$ of the homogeneous in-arborescence network, the ratio

$$
\Delta(n, \Phi, \gamma, \mathscr{K})=\frac{\max _{0.5 \leqslant \frac{\delta}{g} \leqslant 1}\{\xi\}-\min _{0.5 \leqslant \frac{\mathscr{\xi}}{g} \leqslant 1}\{\xi\}}{\max _{\text {entire range of } \mathscr{E}}\{\xi\}-\min _{\text {entire range of } \mathscr{E}}\{\xi\}}
$$

that provides the maximum percentage of the total change of the vulnerability index that occurred within this range of $\frac{\mathscr{E}}{\mathscr{I}}$. If the growth of $\xi$ with respect to $\mathscr{E} / \mathscr{I}$ was uniform or near uniform over the entire range of $\frac{\mathscr{E}}{\mathscr{J}}, \Delta$ would be approximately $\delta=\frac{1-0.5}{3.5-0.25} \approx 0.16$; thus, any value of $\Delta$ significantly higher than $\delta$ indicates a sharp transition within the above-mentioned range of $\mathscr{E} / \mathscr{I}$. As Table 11 shows, when $\gamma=\Phi / 2$ a significant majority of the entries are coordinated shocks and many entries under idiosyncratic shocks are at least $2 \delta$. 


\section{DASGUPTA AND KALIGOUNDER}

Table 10. Values of $\Lambda(n, \Phi, E / I, \mathscr{K})=\frac{\max _{0.05 \leqslant \gamma \leqslant 0.2}\{\xi\}-\min _{0.05 \leqslant \gamma \leqslant 0.2}\{\xi\}}{\max _{\text {entire range of } \gamma}\{\xi\}-\min _{\text {entire range of } \gamma}\{\xi\}}$ for homogeneous dense ER and SF networks under coordinated shocks. Entries that are at least $2 \times 0.375$ are shown in boldface.

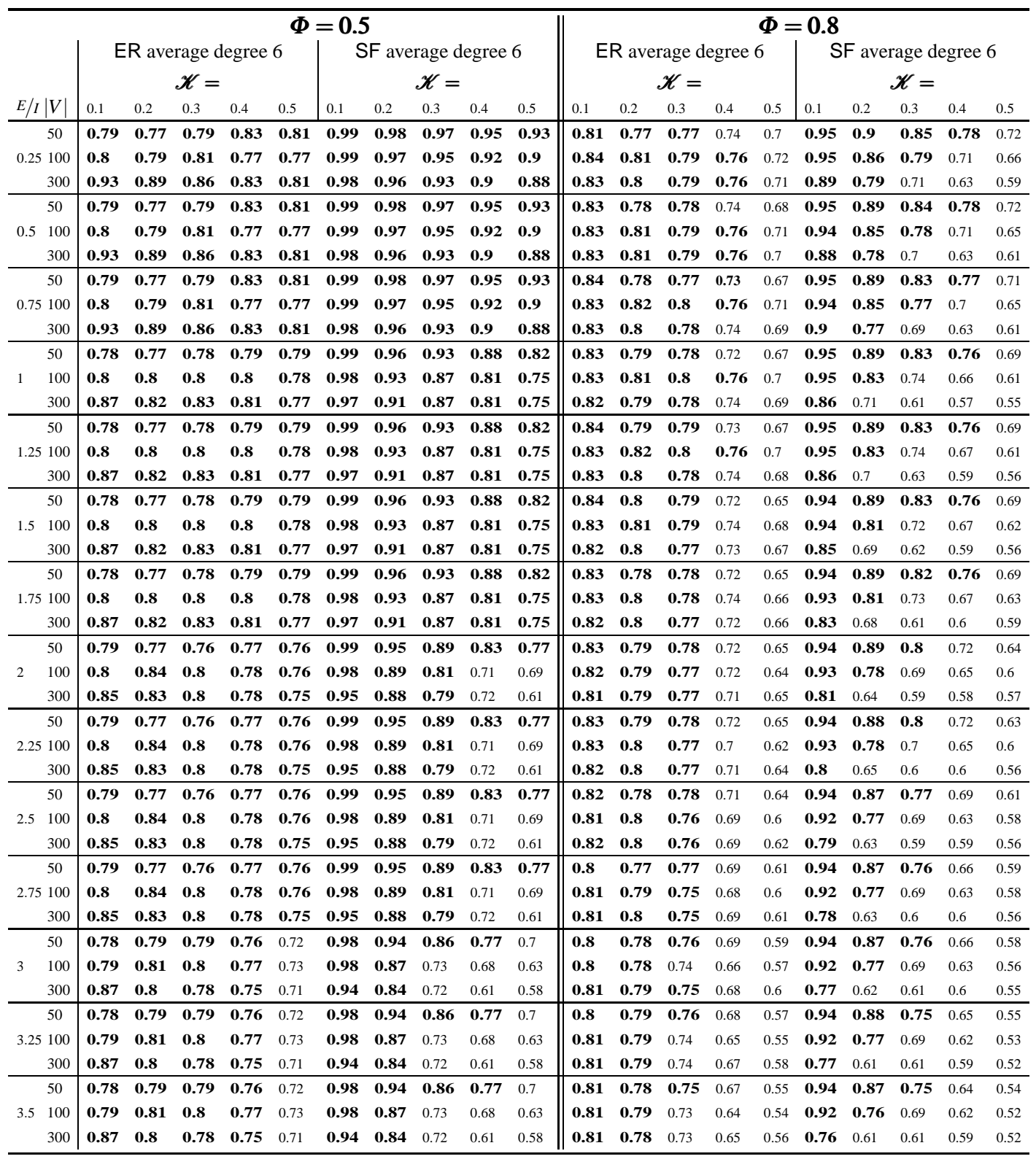


Table 11. Values of $\Delta(n, \Phi, \gamma, \mathscr{K})=\frac{\max _{0.5 \leqslant E / I \leqslant 1}\{\xi\}-\min _{0.5 \leqslant E / I \leqslant 1}\{\xi\}}{\max _{\text {entire range of } E}\{\xi\}-\min _{\text {entire range of } E}\{\xi\}}$ for homogeneous in-arborescence networks. Entries that are at least $2 \times 0.16$ are shown in boldface black. Entries that are at least $\frac{3}{2} \times 0.16$ are shown in boldface gray.

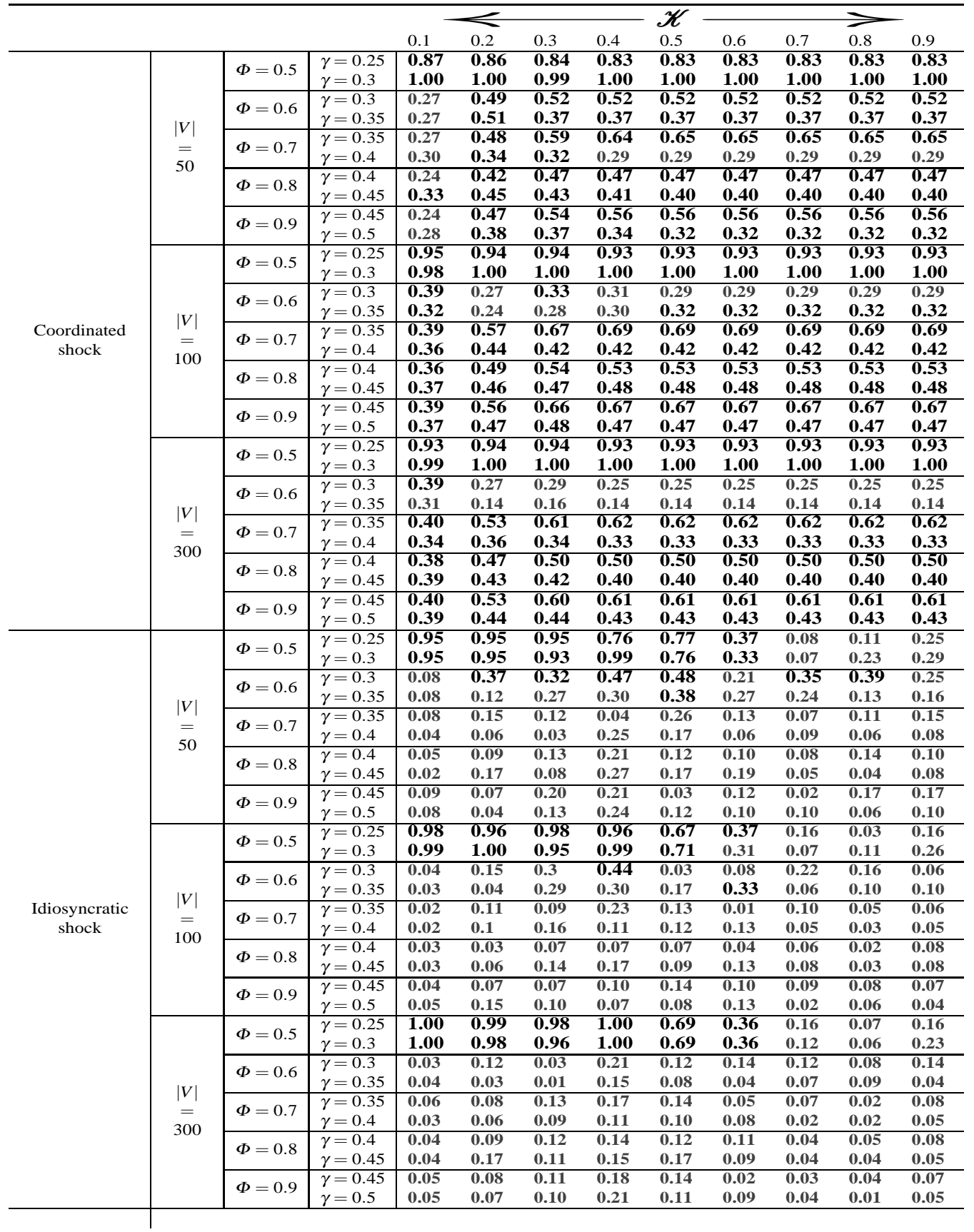


DASGUPTA AND KALIGOUNDER

\section{Formal intuition}

A formal intuition behind such a sharp decrease of $\xi$ can be provided as follows.

Lemma 7.6 (see Section Fof the appendix for a proof) Fix $\gamma, \Phi, \mathscr{I}$, a homogeneous in-arborescence network $G$ and assume that $\gamma \approx \Phi / 2$. Consider any node $v \in V_{\mathbf{X}}$ with $\operatorname{deg}^{\operatorname{in}}(v)>1$, suppose that $v$ fails due to the initial shock. Let $u$ be any node such that $u$ is a "leaf node" (i.e., $\left.\operatorname{deg}^{\text {in }}(u)=0\right)$, and $(u, v) \in E$. Then, as the total external asset $\mathscr{E}$ of the network is varied, there exists a threshold value $\mathscr{E}_{\tau}(u)$ such that

- if $\mathscr{E}<\mathscr{E}_{\tau}(u)$ then $u$ will become insolvent, but

- if $\mathscr{E}>\mathscr{E}_{\tau}(u)$ then $u$ will not become insolvent at any time $t \geqslant 1$, and the shock will not propagate any further through $u$.

The next lemma provides a lower bound, using the degree distributions of the Barábasi-Albert preferential-attachment model [8], on the expected value of the number of leaves in a random inarborescence network for which Lemma 7.6 can be applied.

LEMma 7.7 (see Section Gof the appendix for a proof) Consider a random in-arborescence $G=(V, E)$ generated by the Barábasi-Albert preferential-attachment algorithm [8] as outlined in Section 6.1] and let

$$
\widehat{V}=\left\{u \in V \mid\left(\operatorname{deg}^{\text {in }}(u)=0\right) \wedge\left(\exists v:\left(\left(\operatorname{deg}^{\text {in }}(v)>1\right) \wedge((u, v) \in E)\right)\right)\right\}
$$

Then, $\mathbb{E}[|\widehat{V}|] \geqslant \frac{n}{8}-\frac{11}{8}$.

Let $\xi(\mathscr{E})$ be the value of $\xi$ parameterized by $\mathscr{E}$ (keeping all other parameters unchanged), and let

$$
\begin{aligned}
& \mathscr{E}_{\tau_{\min }}=\min \left\{\mathscr{E}_{\tau}(u) \mid \operatorname{deg}^{\mathrm{in}}(u)=0,(u, v) \in E \text { and } \operatorname{deg}^{\mathrm{in}}(v)>1\right\} \\
& \mathscr{E}_{\tau_{\max }}=\max \left\{\mathscr{E}_{\tau}(u) \mid \operatorname{deg}^{\mathrm{in}}(u)=0,(u, v) \in E \text { and } \operatorname{deg}^{\mathrm{in}}(v)>1\right\}
\end{aligned}
$$

It then follows that

$$
\mathbb{E}\left[\xi\left(\mathscr{E}_{\tau_{\min }}\right)\right]-\mathbb{E}\left[\xi\left(\mathscr{E}_{\tau_{\max }}\right)\right] \geqslant \frac{\frac{n}{8}-\frac{11}{8}}{n} \approx \frac{1}{8}
$$

and $\xi(\mathscr{E})$ exhibits a sharp decrease around the range $\left[\mathscr{E}_{\tau_{\min }}, \mathscr{E}_{\tau_{\max }}\right]$. In practice, the extent of this decrease is expected to be much more than the pessimistic lower bound of $1 / 8$, as our simulation results clearly show.

\section{Concluding remarks}

In this paper, we have initiated a methodology for systematic investigation of the global stabilities of financial networks that arise in OTC derivatives market and elsewhere. Our results can be viewed as a much needed beginning of a systematic investigation of these issues, with future research works concentrating on further improving the network model, the stability measure and parameter choices. 
STABILITY OF FINANCIAL NETWORKS

\section{Acknowledgment}

This work partially supported by NSF grant IIS-1160995.

\section{REFERENCES}

1. Acemoglu D., Ozdaglar A. \& Tahbaz-Salehi A. (2013). Systemic risk and stability in financial networks. working paper.

2. Allen F. \& Babus A. (2009). Networks in Finance, in: Network-based Strategies and Competencies, Kleindorfer P., Wind J. (Eds.), Wharton School Publishing, 367-382.

3. Allen F. \& Gale D. (2000). Financial Contagion. Journal of Political Economy, 108 (1), 1-33.

4. Alon N. \& Spencer J. H. (1992). The Probabilistic Method. John Wiley \& Sons, Inc.

5. Amini H., Cont R. \& MincA A. (2011). Resilience to contagion in financial networks, arXiv:1112.5687v1.

6. BABUS A. (2007a). The Formation of Financial Networks, Tinbergen Institute Discussion Paper No. 2006093/2; FEEM Working Paper No. 69.2007.

7. Babus A. (2007b). Contagion Risk in Financial Networks, in: Financial Development, Integration and Stability, Liebscher K., Christl J., Mooslechner P., Ritzberger-Grunwald D. (Eds.), Edward Elgar Pub, 423-440.

8. BARÁBASI A.-L. \& Albert R. (1999). Emergence of Scaling in Random Networks. Science, 286, 509-512.

9. Berman P., DasGupta B., Kaligounder L. \& Karpinski M. (2013). On Systemic Stability of Banking Networks, Algorithmica, in press.

10. BollobÁs B. (2001). Random Graphs. Cambridge University Press.

11. Bollobás B., Borgs C., Chayes T. \& Riordan O. (2003). Directed scale-free graphs, in: $14^{\text {th }}$ ACMSIAM Symposium on Discrete Algorithms, Society for Industrial and Applied Mathematics, Philadelphia, PA, 132-139.

12. Borodin A. \& El-YAniv R. (1998). Online Computation and Competitive Analysis, Cambridge University Press.

13. Borodin A., Filmus Y. \& OREN J. (2010). Threshold models for competitive influence in social networks, $6^{\text {th }}$ international conference on Internet and network economics, Springer-Verlag Berlin, Heidelberg, 539550.

14. Calabria M. A. (2009). Did Deregulation Cause the Financial Crisis?, Cato Institute.

15. Callaway D. S., Newman M. E. J., Strogatz S. H. \& Watts D. J. (2000). Network robustness and fragility: percolation on random graphs. Physical Review Letters, 85, 5468-5471.

16. Castiglionesi F. \& Navarro B. (2007). Optimal Fragile Financial Networks. Tilburg University Discussion Paper no. 2007-100 (Tilburg, The Netherlands: Tilburg University).

17. CHEN N. (2008). On the approximability of influence in social networks, in: $19^{\text {th }}$ ACM-SIAM Symposium on Discrete Algorithms, Society for Industrial and Applied Mathematics, Philadelphia, PA, 1029-1037.

18. CHEN W., WANG Y. \& YANG S. (2009). Efficient influence maximization in social networks, in: $15^{\text {th }}$ ACM SIGKDD international conference on Knowledge discovery and data mining, Association for Computing Machinery, New York, 199-208.

19. Coelho F. C., Cruz O. G. \& Codeo C. T. (2008). Epigrass: a tool to study disease spread in complex networks. Source Code for Biology and Medicine, 3 (3), 2008.

20. CONT R. (2010). Credit default swaps and financial stability. Finacial Stability Review (Banque de France), 14, 35-43.

21. Cont R., Moussa A. \& Santos E. B. (2010). Network Structure and Systemic Risk in Banking Systems. Working paper.

22. Corbo J. \& Demange G. (2010). in: $3^{\text {rd }}$ Financial Risks International Forum, Paris, France.

23. DAsGupta B . (2004). Capital Accumulation in the Presence of Informal Credit Contracts: Does the Incentive Mechanism Work Better than Credit Rationing Under Asymmetric Information?, Working papers 2004-32, University of Connecticut, Department of Economics.

24. Diamond D. W. \& Dybvig P. H. (1983). Bank runs, deposit insurance and liquidity. Journal of Political Economy, 91, 401-419. 


\section{DASGUPTA AND KALIGOUNDER}

25. EвоLi M. (2004). Systemic Risk in Financial Network: a Graph Theoretical Approach. Mimeo, Univ. di Chieti, Pescara.

26. Elliott M., Golub B. \& Jackson M. O. (2013). Financial networks and contagion. Working paper.

27. Ekelund R. \& Thornton M. (2008). More Awful Truths About Republicans. Ludwig von Mises Institute.

28. EubAnk S. (2005). Network based models of infectious disease spread. Japanese Journal of Infectious Diseases, 58, S19-S13.

29. Eubank S., Guclu H., Kumar V. S. A., Marathe M. V., Srinivasan A., Toroczkai Z. \& Wang N. (2004). Modeling Disease Outbreaks in Realistic Urban Social Networks. Nature, 429, 180-183.

30. FISHER I. (1933). The Debt-Deflation Theory of Great Depressions. Econometrica, 1, 337-357.

31. FreiXas X., PARigi B. M. \& Rochet J.-C. (2000). Systemic risk, interbank relations, and liquidity provision by the central bank. Journal of Money, Credit and Banking, 32 (3), 611-638.

32. Furfine C. H. (2003). Interbank exposures: Quantifying the risk of contagion. Journal of Money, Credit and Banking, 35 (1), 111-128.

33. Gai P. \& Kapadia S. (2010). Contagion in financial networks. Proc. R. Soc. A, 466 (2120), 2401-2423.

34. Haldane A. (2009). Why Banks Failed the Stress Test. speech given at the Marcus-Evans Conference on Stress-Testing, 9-10 February 2009 (available at http://www.bankofengland.co.uk/publications/speeches/2009/speech374.pdf).

35. Haldane A. G. \& MAY R. M. (2011). Systemic risk in banking ecosystems. Nature, 469, 351-355.

36. Hann J. \& Kamber M. (2000). Data Mining: Concepts and Techniques. Morgan Kaufman Publishers.

37. HÜBSCH A. \& Walther U. (2012). The impact of network inhomogeneities on contagion and system stability, CPQF Working Paper Series, No. 32, Frankfurt School of Finance \& Management.

38. IAzZetta C. \& Manna M. (2009). The Topology of the Interbank Market Developements in Italy Since 1990, Bank of Italy Temi di Discussione (Working Paper) No. 711.

39. IYER R. \& PEYDRO J. L. (2010). Interbank Contagion at Work: Evidence from a Natural Experiment. Review of Financial Studies, 24 (4), 1337-1377.

40. Jones C. M. \& Zhigljavsky A. A. (2004). Approximating the negative moments of the Poisson distribution. Statistics \& Probability Letters, 66 (2), 171-181.

41. Kempe D., Kleinberg J. \& TARdos E. (2003). Maximizing the spread of influence through a social network, $9^{\text {th }}$ ACM SIGKDD international conference on Knowledge discovery and data mining, Association for Computing Machinery, New York, 137-146.

42. Keynes J. M. (1936). The General Theory of Employment, Interest, and Money. New York: Harcourt, Brace and Company.

43. Kleindorfer P. R., Wind Y. \& Gunther R. E. (2009). The Network Challenge: Strategy, Profit and Risk in an Interlinked World. Pearson Prentice Hall.

44. Knuth D. E. (1997). The Art of Computer Programming (Fundamental Algorithms), Volume 1, Third Edition, Reading, Massachusetts: Addison-Wesley.

45. LADLEY D. (2013). Contagion and risk sharing on the inter-bank market, Journal of Economics Dynamics \& Control, 37, 1384-1400.

46. LAgunoff R. \& Schreft S. (2001). A Model of Financial Fragility. Journal of Economic Theory, 99 (1-2), 220-264.

47. Liedorp F. R., Medema L., Koetter M., Koning R. H. \& Lelyveld I. van (2010). Peer monitoring or contagion? Interbank market exposure and bank risk, DNB working paper No. 248.

48. Markose S., Giansante S., Gatkowski M. \& Shaghaghi A. R. (2009). Too Interconnected To Fail: Financial Contagion and Systemic Risk In Network Model of CDS and Other Credit Enhancement Obligations of US Banks. Economics Discussion Papers 683, Department of Economics, University of Essex.

49. May R. \& ARinaminpathy N. (2010). Systemic risk: the dynamics of model banking systems. J. R. Soc. Interface, 7, 823-838.

50. MincA A. (2011). Mathematical modeling of default contagion. PhD thesis, Universite Paris VI (Pierre et Marie Curie).

51. Minoiu C. \& Reyes J. A. (2013). A Network Analysis of Global Banking: 1978-2010. Journal of Financial 


\section{STABILITY OF FINANCIAL NETWORKS}

Stability (in press).

52. MinsKY H. (1977). A Theory of Systemic Fragility, in: Financial Crises: Institutions and Markets in a Fragile Environment, Altman E. I., Sametz A. W. (Eds.), Wiley.

53. Mistrulli P. E. (2007). Assessing financial contagion in the interbank market: Maximum entropy versus observed interbank lending patterns. Bank of Italy Research.

54. Moussa A. (2011). Thesis, Columbia University.

55. Nier E., Yang J., Yorulmazer T. \& Alentorn A. (2007). Network models and financial stability. Journal of Economics Dynamics and Control, 31, 2033-2060.

56. ONNELA K.-P., KASKI K. \& KERTÉSZ J. (2004). Clustering and information in correlation based financial networks. Eur. Phys. J. B, 38, 353-362.

57. Rozanov Y. A. (1977). Probability Theory: A Concise Course. Dover Publications, Inc.

58. SACHS A. (2013). Completeness interconnectedness and distribution of interbank exposures - a parameterized analysis of the stability of financial networks. Quantitative Finance (in press).

59. SAntos E. B. \& ConT R. (2010). The Brazilian Interbank Network Structure and Systemic Risk. Working Papers Series 219, Central Bank of Brazil.

60. SaramäKi J., Onnela J.-P., KertéSZ J. \& KASKi K. (2005). Characterizing Motifs in Weighted Complex Networks, in: Science of Complex Networks, Mendes J. F. F. et al. (Eds.).

61. Stauffer D. \& Aharony A. (1994). Introduction to Percolation Theory, 2nd edition, CRC Press.

62. Staum J. C. \& LiU M. (2012). Systemic Risk Components in a Network Model of Contagion. Working paper, Department of Industrial Engineering and Management Sciences, Northwestern University.

63. UPPER C. \& WORMS A. (2004). Estimating bilateral exposures in the german interbank market: Is there a danger of contagion?. European Economic Review, 48 (4), 827-849.

64. ZAWADOWSKI A. (2011). Entangled Financial Systems. Working paper, Boston University School of Management.

\section{APPENDIX}

\section{A. Proof of Lemma 7.1}

We will reuse the notations in Definition 6.1. Using Equation (5.1), for every edge $(u, v) \in E$, the amount of shock received by node $u$ at time $t=1$ is as follows:

- If $G$ is homogeneous then

$$
\begin{aligned}
\mathbb{E}\left[\Delta_{\text {homo }}(u)\right] & =\frac{\min \left\{\Phi\left(\operatorname{deg}^{\text {in }}(v)-\operatorname{deg}^{\text {out }}(v)+\frac{\mathscr{E}}{n}\right)-\gamma\left(\operatorname{deg}{ }^{\text {in }}(v)+\frac{\mathscr{E}}{n}\right), \operatorname{deg}^{\text {in }}(v)\right\}}{\operatorname{deg}^{\text {in }}(v)} \\
& =\min \left\{(\Phi-\gamma)+\frac{\frac{\mathscr{E}}{n}(\Phi-\gamma)-\Phi \operatorname{deg}^{\text {out }}(v)}{\operatorname{deg}^{\text {in }}(v)}, 1\right\}
\end{aligned}
$$

- If $G$ is $(\alpha, \beta)$-heterogeneous, then $\sigma_{v}=\frac{\beta}{\alpha}$ and, using linearity of expectation, we get

$$
\begin{aligned}
\mathbb{E}\left[b_{v}\right] & =\mathbb{E}\left[\sum_{(u, v) \in \widetilde{E}}\left(\alpha \frac{\beta \mathscr{I}}{\alpha|\widetilde{E}|}+(1-\alpha) \frac{(1-\beta) \mathscr{I}}{|E|-\alpha|\widetilde{E}|}\right)\right] \\
& =\operatorname{deg}^{\text {in }}(v) \mathbb{E}\left[\left(\beta \frac{\mathscr{I}}{|\widetilde{E}|}+(1-\alpha) \frac{(1-\beta) \mathscr{I}}{|E|-\alpha|\widetilde{E}|}\right)\right]
\end{aligned}
$$




$$
\begin{aligned}
& \mathbb{E}\left[b_{v}-u_{v}\right]=\mathbb{E}\left[\sum_{(u, v) \in \widetilde{E}}\left(\alpha \frac{\beta \mathscr{I}}{\alpha|\widetilde{E}|}+(1-\alpha) \frac{(1-\beta) \mathscr{I}}{|E|-\alpha|\widetilde{E}|}\right)-\sum_{(v, u) \in \widetilde{E}}\left(\alpha \frac{\beta \mathscr{I}}{\alpha|\widetilde{E}|}+(1-\alpha) \frac{(1-\beta) \mathscr{I}}{|E|-\alpha|\widetilde{E}|}\right)\right] \\
& =\sum_{(u, v) \in \widetilde{E}} \mathbb{E}\left[\left(\beta \frac{\mathscr{I}}{|\widetilde{E}|}+(1-\alpha) \frac{(1-\beta) \mathscr{I}}{|E|-\alpha|\widetilde{E}|}\right)\right]-\sum_{(v, u) \in \widetilde{E}} \mathbb{E}\left[\left(\beta \frac{\mathscr{I}}{|\widetilde{E}|}+(1-\alpha) \frac{(1-\beta) \mathscr{I}}{|E|-\alpha|\widetilde{E}|}\right)\right] \\
& =\left(\operatorname{deg}^{\text {in }}(v)-\operatorname{deg}^{\text {out }}(v)\right) \mathbb{E}\left[\left(\beta \frac{\mathscr{I}}{|\widetilde{E}|}+(1-\alpha) \frac{(1-\beta) \mathscr{I}}{|E|-\alpha|\widetilde{E}|}\right)\right]
\end{aligned}
$$$$
\mathbb{E}\left[\Delta_{\text {hetero }}(u)\right]=\mathbb{E}\left[\frac{\min \left\{\Phi\left(b_{v}-\imath_{v}+\sigma_{v} \mathscr{E}\right)-\gamma\left(b_{v}+\sigma_{v} \mathscr{E}\right), b_{v}\right\}}{\operatorname{deg}^{\operatorname{in}}(v)}\right]
$$$$
=\min \left\{\mathbb{E}\left[\frac{\Phi\left(b_{v}-\imath_{v}+\sigma_{v} \mathscr{E}\right)-\gamma\left(b_{v}+\sigma_{v} \mathscr{E}\right)}{\operatorname{deg}{ }^{\text {in }}(v)}\right], \mathbb{E}\left[\frac{b_{v}}{\operatorname{deg}^{\operatorname{in}}(v)}\right]\right\}
$$$$
=\min \left\{\frac{\Phi}{\operatorname{deg}^{\operatorname{in}(v)}} \mathbb{E}\left[b_{v}-u_{v}\right]+\frac{\Phi \sigma_{v} \mathscr{E}}{\operatorname{deg}^{\operatorname{in}}(v)}-\frac{\gamma}{\operatorname{deg}^{\operatorname{in}}(v)} \mathbb{E}\left[b_{v}\right]-\frac{\gamma \sigma_{v} \mathscr{E}}{\operatorname{deg}^{\operatorname{in}}(v)}, \frac{1}{\operatorname{deg}^{\operatorname{in}(v)}} \mathbb{E}\left[b_{v}\right]\right\}
$$$$
=\min \left\{\frac{\Phi}{\operatorname{deg}^{\operatorname{in}(v)}}\left(\operatorname{deg}^{\mathrm{in}}(v)-\operatorname{deg}^{\text {out }}(v)\right) \mathbb{E}\left[\left(\beta \frac{\mathscr{I}}{|\widetilde{E}|}+(1-\alpha) \frac{(1-\beta) \mathscr{I}}{|E|-\alpha|\widetilde{E}|}\right)\right]+\frac{\Phi \sigma_{v} \mathscr{E}}{\operatorname{deg}^{\mathrm{in}}(v)}\right.
$$$$
-\frac{\gamma}{\operatorname{deg}^{\operatorname{in}(v)}} \operatorname{deg}^{\operatorname{in}}(v) \mathbb{E}\left[\left(\beta \frac{\mathscr{I}}{|\widetilde{E}|}+(1-\alpha) \frac{(1-\beta) \mathscr{I}}{|E|-\alpha|\widetilde{E}|}\right)\right]-\frac{\gamma \sigma_{v} \mathscr{E}}{\operatorname{deg}^{\operatorname{in}}(v)},
$$$$
\left.\mathbb{E}\left[\left(\beta \frac{\mathscr{I}}{|\widetilde{E}|}+(1-\alpha) \frac{(1-\beta) \mathscr{I}}{|E|-\alpha|\widetilde{E}|}\right)\right]\right\}
$$$$
=\min \left\{\frac{\Phi\left(\operatorname{deg}^{\text {in }}(v)-\operatorname{deg}^{\text {out }}(v)\right)-\gamma \operatorname{deg}^{\text {in }}(v)}{\operatorname{deg}^{\text {in }}(v)} \mathbb{E}\left[\left(\beta \frac{\mathscr{I}}{|\widetilde{E}|}+(1-\alpha) \frac{(1-\beta) \mathscr{I}}{|E|-\alpha|\widetilde{E}|}\right)\right]+\frac{(\Phi-\gamma) \sigma_{v} \mathscr{E}}{\operatorname{deg}^{\text {in }}(v)},\right.
$$

$$
\begin{aligned}
& \left.\mathbb{E}\left[\left(\beta \frac{\mathscr{I}}{|\widetilde{E}|}+(1-\alpha) \frac{(1-\beta) \mathscr{I}}{|E|-\alpha|\widetilde{E}|}\right)\right]\right\} \\
& \geqslant \min \left\{\frac{\Phi\left(\operatorname{deg}^{\text {in }}(v)-\operatorname{deg}^{\text {out }}(v)\right)-\gamma \operatorname{deg}^{\text {in }}(v)}{\operatorname{deg}^{\text {in }}(v)} \beta \mathbb{E}\left[\frac{\mathscr{L}}{|\widetilde{E}|}\right]+\frac{(\Phi-\gamma) \sigma_{v} \mathscr{E}}{\operatorname{deg}^{\text {in }}(v)}, \beta \mathbb{E}\left[\frac{\mathscr{G}}{|\widetilde{E}|}\right]\right\}
\end{aligned}
$$

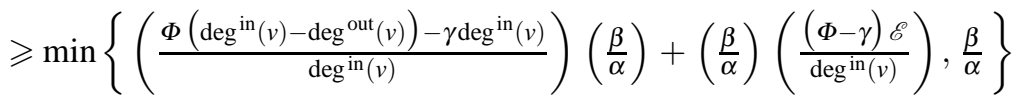

$$
\begin{aligned}
& \text { since } \mathbb{E}\left[\frac{\mathscr{q}}{|\widetilde{E}|}\right]=\mathbb{E}\left[\frac{|E|}{|\widetilde{E}|}\right]=\alpha \\
& \geqslant \frac{\beta}{\alpha} \min \left\{(\Phi-\gamma)+\frac{(\Phi-\gamma) \mathscr{E}-\Phi \operatorname{deg}^{\text {out }}(v)}{\operatorname{deg}^{\text {in }}(v)}, 1\right\} \\
& \geqslant \frac{\beta}{\alpha} \mathbb{E}\left[\Delta_{\text {homo }}(u)\right]
\end{aligned}
$$


STABILITY OF FINANCIAL NETWORKS

\section{B. Proof of Lemma 7.2}

Using standard probabilistic calculations, we get

$$
\begin{aligned}
& \mathbb{E}\left[\frac{1}{\operatorname{deg} \text { in }(v)} \mid \operatorname{deg}^{\text {in }}(v)>0\right]=\sum_{k=1}^{n-1} \frac{1}{k} \operatorname{Pr}\left[\operatorname{deg}^{\text {in }}(v)=k\right]=C \sum_{k=1}^{n-1} k^{-4} \approx C \zeta(4) \approx \frac{\pi^{2}}{15} \operatorname{deg}_{\text {ave }} \\
& \operatorname{Var}\left[\operatorname{deg}^{\text {in }}(v)\right]=\mathbb{E}\left[\left(\operatorname{deg}^{\text {in }}(v)\right)^{2}\right]-\left(\mathbb{E}\left[\operatorname{deg}^{\text {in }}(v)\right]\right)^{2}=\sum_{k=1}^{n-1} k^{2}\left(C k^{-3}\right)-\left(\operatorname{deg}_{\text {ave }}\right)^{2} \\
& =C \sum_{k=1}^{n-1} \frac{1}{k}-\left(\operatorname{deg}_{\mathrm{ave}}\right)^{2} \approx \frac{6 \operatorname{deg}_{\mathrm{ave}}}{\pi^{2}} \ln n-\left(\operatorname{deg}_{\mathrm{ave}}\right)^{2} \approx \frac{6 \operatorname{deg}_{\mathrm{ave}}}{\pi^{2}} \ln n
\end{aligned}
$$

\section{Proof of Lemma 7.3}

Let $\mathscr{D}=\sum_{v \in \widetilde{V}} \operatorname{deg}{ }^{\text {in }}(v)+\sum_{v \in \widetilde{V}} \operatorname{deg}$ out $(v)$. By linearity of expectation, we have

$$
\mathbb{E}[\mathscr{D}]=2 \sum_{v \in \widetilde{V}} \mathbb{E}\left[\operatorname{deg}^{\text {in }}(v)\right]=2 \alpha n \operatorname{deg}_{\text {ave }}
$$

and similarly, since $\operatorname{deg}^{\text {in }}(v)$ is independent of any other $\operatorname{deg}^{\text {in }}(u)$ for $u \neq v$, we have

$$
\operatorname{Var}[\mathscr{D}]=2 \sum_{v \in \widetilde{V}} \operatorname{Var}\left[\operatorname{deg}^{\text {in }}(v)\right] \approx \frac{12 \operatorname{deg}_{\text {ave }}}{\pi^{2}} \alpha n \ln n
$$

Thus, via Chebyschev's inequality [4, page 37], for any positive $\lambda$ we have

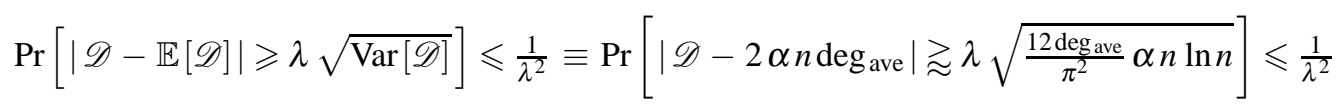

Setting $\lambda=\sqrt{\frac{\pi^{2} \ln n}{12 \alpha}}$ gives $\operatorname{Pr}\left[\left|\mathscr{D}-2 \alpha n \operatorname{deg}_{\text {ave }}\right| \gtrsim \sqrt{n \operatorname{deg}_{\text {ave }}} \ln n\right] \leqslant \frac{12 \alpha}{\pi^{2} \ln n}$ and thus w.h.p. $\mathscr{D} \approx$ $2 \alpha n \operatorname{deg}_{\text {ave. }}$ Since $\frac{\mathscr{D}}{2} \leqslant|\widetilde{E}| \leqslant \mathscr{D}$, it now follows that

$$
\begin{array}{r}
\alpha n \operatorname{deg}_{\text {ave }} \leqslant \mathbb{E}[|\widetilde{E}|] \leqslant 2 \alpha n \operatorname{deg}_{\text {ave }} \\
\text { w.h.p. } \alpha n \operatorname{deg}_{\text {ave }} \leqslant \quad|\widetilde{E}| \leqslant 2 \alpha n \operatorname{deg}_{\text {ave }}
\end{array}
$$

Also, note that $\operatorname{Pr}[(u, v) \in \widetilde{E}]=\operatorname{Pr}[v \in \widetilde{V}]=\alpha$. For notational convenience, let $\widetilde{E}_{1}$ be a random subset of $\alpha|\widetilde{E}|$ of edges from the edges in $\widetilde{E}$ as used in Definition 6.1. This implies that

$$
\begin{aligned}
& \mathbb{E}\left[c_{1}^{\text {hetero }}\right]=\operatorname{Pr}\left[(u, v) \in \widetilde{E}_{1}\right]\left(\frac{\beta \mathscr{I}}{\alpha|\widetilde{E}|}\right)+\left(1-\operatorname{Pr}\left[(u, v) \in \widetilde{E}_{1}\right]\right) \frac{\mathscr{I}-\beta \mathscr{I}}{|E|-\alpha|\widetilde{E}|} \\
&=\alpha \operatorname{Pr}[(u, v) \in \widetilde{E}]\left(\frac{\beta \mathscr{I}}{\alpha|\widetilde{E}|}\right)+(1-\alpha \operatorname{Pr}[(u, v) \in \widetilde{E}]) \frac{\mathscr{I}-\beta \mathscr{I}}{|E|-\alpha|\widetilde{E}|} \\
&=\alpha^{2}\left(\frac{\beta \mathscr{I}}{\alpha|\widetilde{E}|}\right)+\left(1-\alpha^{2}\right) \frac{\mathscr{I}-\beta \mathscr{I}}{|E|-\alpha|\widetilde{E}|} \\
& \Rightarrow \mathrm{w} . \mathrm{h} . \mathrm{p} \cdot \quad \alpha^{2} \frac{\beta n \operatorname{deg}_{\mathrm{ave}}}{2 \alpha n \operatorname{deg}_{\mathrm{ave}}}+\left(1-\alpha^{2}\right) \frac{(1-\beta) n \operatorname{deg}_{\text {ave }}}{n \operatorname{deg}_{\text {ave }}-\alpha n \operatorname{deg}_{\text {ave }}}
\end{aligned}
$$


DASGUPTA AND KALIGOUNDER

$$
\begin{array}{r}
\qquad \mathbb{E}\left[c_{1}^{\text {hetero }}\right] \leqslant \alpha^{2} \frac{\beta n \operatorname{deg}_{\text {ave }}}{\alpha n \operatorname{deg}_{\text {ave }}}+\left(1-\alpha^{2}\right) \frac{(1-\beta) n \operatorname{deg}_{\text {ave }}}{n \operatorname{deg}_{\text {ave }}-2 \alpha n \text { deg ave }_{\text {ave }}} \\
\equiv \mathrm{w} . \mathrm{h} \cdot \mathrm{p} \cdot \quad \frac{\alpha \beta}{2}+(1+\alpha)(1-\beta) \leqslant \mathbb{E}\left[c_{1}^{\text {hetero }}\right] \leqslant \alpha \beta+\frac{\left(1-\alpha^{2}\right)(1-\beta)}{1-2 \alpha} \\
\equiv \mathrm{w} . \mathrm{h} \cdot \mathrm{p} \cdot \quad 1+\alpha-\beta-\frac{\alpha \beta}{2} \leqslant \mathbb{E}\left[c_{1}^{\text {hetero }}\right] \leqslant \frac{1+\alpha \beta-\alpha^{2}-\beta-\alpha^{2} \beta}{1-2 \alpha}
\end{array}
$$

\section{Proof of Lemma 7.4}

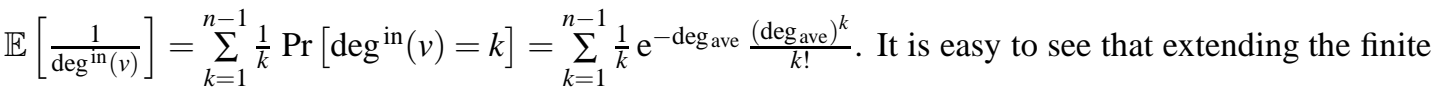
series to an infinite series does not change the asymptotic value of the series since

$$
\begin{aligned}
& \sum_{k=1}^{\infty} \frac{1}{k} \mathrm{e}^{-\operatorname{deg}_{\text {ave }}} \frac{\left(\text { deg }_{\text {ave }}\right)^{k}}{k !}-\sum_{k=1}^{n-1} \frac{1}{k} \mathrm{e}^{-\operatorname{deg}_{\text {ave }}} \frac{\left(\text { deg }_{\text {ave }}\right)^{k}}{k !} \\
& =\sum_{k=n}^{\infty} \frac{1}{k} \mathrm{e}^{-\operatorname{deg}_{\mathrm{ave}}} \frac{\left(\operatorname{deg}_{\mathrm{ave}}\right)^{k}}{k !} \\
& \leqslant \frac{\mathrm{e}^{- \text {deg ave }}}{n} \sum_{k=n}^{\infty} \frac{\left(\operatorname{deg}_{\mathrm{ave}}\right)^{k}}{k !} \\
& \leqslant\left(\frac{\mathrm{e}^{- \text {deg ave }_{\text {a }}}}{n}\right)\left(\frac{\left(\text { deg }_{\text {ave }}\right)^{n}}{n !}\right)\left(\max _{0 \leqslant x \leqslant \operatorname{deg}_{\text {ave }}}\left\{\mathrm{e}^{x}\right\}\right) \\
& =\left(\frac{\mathrm{e}^{- \text {deg ave }_{\text {a }}}}{n}\right)\left(\frac{\left(\operatorname{deg}_{\text {ave }}\right)^{n}}{n !}\right) \mathrm{e}^{\operatorname{deg}_{\text {ave }}} \\
& =\frac{\left(\operatorname{deg}_{\mathrm{ave}}\right)^{n}}{n(n !)}
\end{aligned}
$$

using the Lagrange remainder term for the Maclaurin series expansion of $\mathrm{e}^{\mathrm{deg}_{\text {ave }}}$

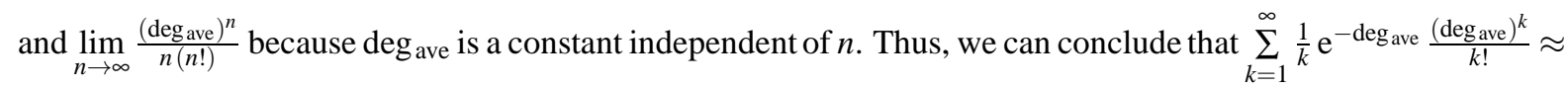
$\sum_{k=1}^{n-1} \frac{1}{k} \mathrm{e}^{-\operatorname{deg}_{\text {ave }}} \frac{\left(\operatorname{deg}_{\text {ave }}\right)^{k}}{k !}$. It now follows that

$$
\begin{aligned}
\frac{\partial}{\partial d} \mathbb{E}\left[\frac{1}{\operatorname{deg}{ }^{\text {in }(v)}} \mid \operatorname{deg}_{\text {ave }}=d\right] \approx \frac{\partial}{\partial d} \sum_{k=1}^{\infty} \frac{1}{k} \mathrm{e}^{-d} \frac{d^{k}}{k !} \\
=\mathrm{e}^{-d} \sum_{k=1}^{\infty} \frac{d^{k-1}}{k !}=\frac{\mathrm{e}^{-d}}{d} \sum_{k=1}^{\infty} \frac{d^{k}}{k !}=\frac{\mathrm{e}^{-d}}{d}\left(\mathrm{e}^{d}-1\right)=\frac{1-\mathrm{e}^{-d}}{d}
\end{aligned}
$$

This proves one of the claims in the lemma. To prove the other claim, using a well-known approximation on the first inverse moment of Poisson's distribution [40, page 173] we have

$$
\left|\sum_{k=1}^{\infty} \frac{1}{k} \mathrm{e}^{-\operatorname{deg}_{\text {ave }}} \frac{\left(\operatorname{deg}_{\text {ave }}\right)^{k}}{k !}-\sum_{k=1}^{\left\lfloor 3 \operatorname{deg}_{\text {ave }}+10\right\rfloor} \frac{1}{k} \mathrm{e}^{-\operatorname{deg}_{\text {ave }}} \frac{\left(\operatorname{deg}_{\text {ave }}\right)^{k}}{k !}\right|<10^{-10}
$$

and therefore we obtain

$$
\left|\sum_{k=1}^{n-1} \frac{1}{k} \mathrm{e}^{- \text {deg }_{\text {ave }}} \frac{\left(\operatorname{deg}_{\text {ave }}\right)^{k}}{k !}-\sum_{k=1}^{\left\lfloor 3 \operatorname{deg}_{\text {ave }}+10\right\rfloor} \frac{1}{k} \mathrm{e}^{- \text {deg }_{\text {ave }}} \frac{\left(\text { deg }_{\text {ave }}\right)^{k}}{k !}\right| \lesssim 10^{-10}
$$




\section{STABILITY OF FINANCIAL NETWORKS}

\section{E. Proof of Lemma 7.5}

Let $\mathscr{D}=\sum_{v \in \widetilde{V}} \operatorname{deg}^{\text {in }}(v)+\sum_{v \in \widetilde{V}} \operatorname{deg}{ }^{\text {out }}(v)$. We can reuse the proof of Lemma 7.3 provided we show that $\mathbb{E}[\mathscr{D}]=2 \alpha n \operatorname{deg}_{\text {ave }}$ and w.h.p. $\mathscr{D} \approx 2 \alpha n \operatorname{deg}_{\text {ave }}$. By linearity of expectation, we have $\mathbb{E}[\mathscr{D}]=$ $2 \sum_{v \in \widetilde{V}} \mathbb{E}\left[\operatorname{deg}^{\text {in }}(v)\right]=2 \alpha n \operatorname{deg}_{\text {ave }}$, and similarly $\operatorname{Var}[\mathscr{D}]=2 \sum_{v \in \widetilde{V}} \operatorname{Var}\left[\operatorname{deg}^{\text {in }}(v)\right]=2 \alpha n\left(1-\frac{\operatorname{deg}_{\text {ave }}}{n-1}\right) \operatorname{deg}_{\text {ave }} \approx$

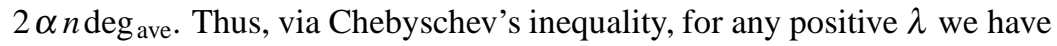

$$
\operatorname{Pr}[|\mathscr{D}-\mathbb{E}[\mathscr{D}]| \geqslant \lambda \sqrt{\operatorname{Var}[\mathscr{D}]}] \leqslant \frac{1}{\lambda^{2}} \equiv \operatorname{Pr}\left[\left|\mathscr{D}-2 \alpha n \operatorname{deg}_{\text {ave }}\right| \gtrsim \lambda \sqrt{2 \alpha n \operatorname{deg}_{\text {ave }}}\right] \leqslant \frac{1}{\lambda^{2}}
$$

Setting $\lambda=\sqrt{\frac{\ln n}{2 \alpha}}$ gives

$$
\operatorname{Pr}\left[\left|\mathscr{D}-2 \alpha n \operatorname{deg}_{\text {ave }}\right| \gtrsim \sqrt{n \operatorname{deg}_{\text {ave }} \ln n}\right] \leqslant \frac{2 \alpha}{\ln n}
$$

and thus w.h.p. $\mathscr{D} \approx 2 \alpha n$ deg $_{\text {ave }}$.

\section{F. Proof of Lemma 7.6}

The amount of shock $\mu$ transmitted from $v$ to $u$ is given by

$$
\mu=\min \left\{(\Phi-\gamma)\left(1+\frac{\mathscr{E}}{n \operatorname{deg}^{\text {in }}(v)}\right)+\Phi \frac{\operatorname{deg}^{\text {out }}(v)}{\operatorname{deg}^{\text {in }}(v)}, 1\right\}
$$

Since $G$ is an in-arborescence, $\operatorname{deg}^{\text {out }}(v) \leqslant 1$. First, consider the case of $\operatorname{deg}^{\text {out }}(v)=0$. In this case, $\mu=\min \left\{(\Phi-\gamma)\left(1+\frac{\mathscr{E}}{n \operatorname{deg}^{\operatorname{in}}(v)}\right), 1\right\}$ and thus we have

$$
c_{u}(1)=c_{u}(0)-\mu=\gamma \frac{\mathscr{E}}{n}-\min \left\{(\Phi-\gamma)\left(1+\frac{\mathscr{E}}{n \operatorname{deg}^{\operatorname{in}(v)}}\right), 1\right\}
$$

Assuming $\gamma \approx \Phi / 2$, we have

$$
\begin{aligned}
c_{u}(1) \approx \gamma \frac{\mathscr{E}}{n}+\operatorname{deg}^{\mathrm{in}}(u)-\min \left\{\gamma\left(1+\frac{\mathscr{E}}{n \operatorname{deg}^{\mathrm{in}}(v)}\right)\right. & , 1\} \\
& =\min \left\{\gamma\left(\frac{\mathscr{E}}{n}-1-\frac{\mathscr{E}}{n \operatorname{deg}^{\mathrm{in}}(v)}\right), \gamma \frac{\mathscr{E}}{n}-1\right\}
\end{aligned}
$$

There are two cases to consider:

- If $\gamma\left(\frac{\mathscr{E}}{n}-1-\frac{\mathscr{E}}{n \operatorname{deg}^{\mathrm{in}}(v)}\right) \geqslant \gamma \frac{\mathscr{E}}{n}-1$ then

$$
c_{u}(1) \approx \gamma\left(\frac{\mathscr{E}}{n}-1-\frac{\mathscr{E}}{n \operatorname{deg}^{\mathrm{in}}(v)}\right)=\gamma\left(\frac{\mathscr{E}}{n}\left(1-\frac{1}{\operatorname{deg}^{\mathrm{in}}(v)}\right)-1\right)
$$

Thus, if $\mathscr{E}>\mathscr{E}_{\tau_{1}}(u)=\frac{n}{1-\frac{1}{\operatorname{deg}^{\operatorname{in}(v)}}}$ then $c_{u}(1)$ would be strictly positive, the node $u$ will not become insolvent at time $t=1$, but if $\mathscr{E}<\mathscr{E}_{\tau_{1}}(u)$ then $c_{u}(1)$ would be strictly negative and $u$ would fail. 
DASGUPTA AND KALIGOUNDER

- Otherwise, $c_{u}(1) \approx \gamma \frac{\mathscr{E}}{n}-1$. Thus, if $\mathscr{E}>\mathscr{E}_{\tau_{2}}(u)=\frac{n}{\mathscr{E}}$ then $c_{u}(1)$ would be strictly positive, the node $u$ will not become insolvent at time $t=1$, but if $\mathscr{E}<\mathscr{E}_{\tau_{1}}(u)$ then $c_{u}(1)$ would be strictly negative and $u$ would fail.

A similar analysis may be carried out if $\operatorname{deg}^{\text {out }}(v)=1$ leading to slightly two different threshold values, say $\mathscr{E}_{\tau_{3}}(u)$ and $\mathscr{E}_{\tau_{4}}(u)$. Since deg ${ }^{\text {out }}(u)=1$, if $u$ does not become insolvent at time $t=1$ then it does not become insolvent for any $t>1$ as well.

\section{G. Proof of Lemma7.7}

Let $r$ be the root node of $G$. Note that, for any node $u \in V \backslash\{r\}, \operatorname{deg}^{\text {out }}(u)=1$. Thus, using the results in [8], it follows that for any node $u \in V \backslash\{r\}, \operatorname{Pr}\left[\operatorname{deg}^{\operatorname{in}}(u)=k-1\right] \propto 1 / k^{3}$ and in particular $\operatorname{Pr}\left[\operatorname{deg}^{\text {in }}(u)=1\right] \leqslant 1 / 4$. For $j=0,2, \ldots, n$, let $n_{j}$ be the number of nodes $u$ of $G$ with $\operatorname{deg}^{\text {in }}(u)=j$. Thus, $n=\sum_{j=0}^{n} n_{j},|E|=n-1=\sum_{j=1}^{n} j n_{j}$, and

$$
\begin{aligned}
\sum_{u \in V \backslash\{r\}} \operatorname{Pr}\left[\operatorname{deg}^{\mathrm{in}}(u)=1\right] & \leqslant \mathbb{E}\left[n_{1}\right] \leqslant 1+\sum_{u \in V \backslash\{r\}} \operatorname{Pr}\left[\operatorname{deg}^{\mathrm{in}}(u)=1\right] \\
& \equiv \frac{n-1}{4} \leqslant \mathbb{E}\left[n_{1}\right] \leqslant 1+\frac{n-1}{4} \equiv \mathbb{E}\left[n_{1}\right]=\frac{n-1}{4}+t \quad \text { for some } t \in[0,1]
\end{aligned}
$$

Letting $n_{>1}=\sum_{j=2}^{n} n_{j}$, we have

$$
\begin{aligned}
& \mathbb{E}\left[n_{0}+n_{>1}\right]=n-\mathbb{E}\left[n_{1}\right]=\frac{3 n+1}{4}-t \\
& \begin{aligned}
\mathbb{E}\left[\sum_{j=1}^{n} j n_{j}\right] & =n-1 \equiv \mathbb{E}\left[n_{1}\right]+\mathbb{E}\left[\sum_{j=2}^{n} j n_{j}\right]=n-1 \\
& \equiv \mathbb{E}\left[n_{1}\right]+2 \mathbb{E}\left[n_{>1}\right] \leqslant n-1 \Rightarrow \mathbb{E}\left[n_{>1}\right] \leqslant \frac{n-1-\frac{3 n+1}{4}+t}{2}=\frac{n}{8}+\frac{4 t-5}{8}
\end{aligned} \\
& \mathbb{E}\left[n_{0}\right]=n-\mathbb{E}\left[n_{1}\right]-\mathbb{E}\left[n_{>1}\right] \geqslant n-\left(\frac{n-1}{4}+t\right)-\left(\frac{n}{8}+\frac{4 t-5}{8}\right)=\frac{n}{8}+\frac{7}{8}-\frac{3 t}{2}
\end{aligned}
$$

and hence we can bound $\mathbb{E}[|\widehat{V}|]$ as

$$
\begin{aligned}
\mathbb{E}[|\widehat{V}|]=\mathbb{E}\left[n_{0}\right]-\mathbb{E}\left[\left|\left\{u \in V \mid\left(\operatorname{deg}^{\mathrm{in}}(u)=0\right) \wedge\left(\exists v:\left(\left(\operatorname{deg}^{\mathrm{in}}(v)=1\right) \wedge((u, v) \in E)\right)\right)\right\}\right|\right] \\
\geqslant \mathbb{E}\left[n_{0}\right]-\mathbb{E}\left[n_{1}\right] \geqslant\left(\frac{n}{8}+\frac{7}{8}-\frac{3 t}{2}\right)-\left(\frac{n-1}{4}+t\right)=\frac{n}{8}+\frac{9}{8}-\frac{5 t}{2} \geqslant \frac{n}{8}-\frac{11}{8}
\end{aligned}
$$


Supplementary documents follow Supplementary Table S1-Table S9

Supplementary color figures FIG. S1-FIG. S11 
DASGUPTA AND KALIGOUNDER

Table S1. Residual instabilities of homogeneous versus heterogeneous networks under idiosyncratic shocks. The percentages shown are the percentages of networks for which $\xi<0.05$ or $\xi<0.1$ or $\xi<0.2$.

\begin{tabular}{|c|c|c|c|c|c|c|c|c|}
\hline & & & & & idiosyncr & tic shock & & \\
\hline & & & $\Phi=$ & $0.5, \gamma=0$ & & $\Phi=$ & $0.5, \gamma=0$ & \\
\hline & & & $\xi<0.05$ & $\xi<0.1$ & $\xi<0.2$ & $\xi<0.05$ & $\xi<0.1$ & $\xi<0.2$ \\
\hline & & in-arborescence & $76 \%$ & 78\% & $82 \%$ & $26 \%$ & $58 \%$ & $75 \%$ \\
\hline & & ER, average degree 3 & $99 \%$ & $100 \%$ & $100 \%$ & $43 \%$ & $84 \%$ & $100 \%$ \\
\hline & homogeneous & ER, average degree 6 & $100 \%$ & $100 \%$ & $100 \%$ & $100 \%$ & $100 \%$ & $100 \%$ \\
\hline & & SF, average degree 3 & $42 \%$ & $100 \%$ & $100 \%$ & $23 \%$ & $70 \%$ & $88 \%$ \\
\hline & & SF, average degree 6 & $100 \%$ & $100 \%$ & $100 \%$ & $100 \%$ & $100 \%$ & $100 \%$ \\
\hline & & in-arborescence & $1 \%$ & $2 \%$ & $15 \%$ & $0 \%$ & $1 \%$ & $10 \%$ \\
\hline & & ER, average degree 3 & $0 \%$ & $2 \%$ & $16 \%$ & $0 \%$ & $1 \%$ & $8 \%$ \\
\hline$|V|=50$ & $(0.1,0.95)$-heterogeneous & ER, average degree 6 & $7 \%$ & $10 \%$ & $21 \%$ & $2 \%$ & $7 \%$ & $14 \%$ \\
\hline & & SF, average degree 3 & $0 \%$ & $6 \%$ & $22 \%$ & $0 \%$ & $2 \%$ & $14 \%$ \\
\hline & & SF, average degree 6 & $8 \%$ & $19 \%$ & $34 \%$ & $4 \%$ & $12 \%$ & $21 \%$ \\
\hline & & in-arborescence & $0 \%$ & $2 \%$ & $12 \%$ & $0 \%$ & $1 \%$ & $11 \%$ \\
\hline & & ER, average degree 3 & $7 \%$ & $14 \%$ & $22 \%$ & $4 \%$ & $9 \%$ & $18 \%$ \\
\hline & $(0.2,0.6)$-heterogeneous & ER, average degree 6 & $8 \%$ & $18 \%$ & $30 \%$ & $6 \%$ & $10 \%$ & $20 \%$ \\
\hline & & SF, average degree 3 & $0 \%$ & $9 \%$ & $19 \%$ & $0 \%$ & $3 \%$ & $17 \%$ \\
\hline & & SF, average degree 6 & $8 \%$ & $12 \%$ & $24 \%$ & $4 \%$ & $7 \%$ & $18 \%$ \\
\hline & & in-arborescence & $76 \%$ & $78 \%$ & $81 \%$ & $32 \%$ & $62 \%$ & $81 \%$ \\
\hline & & ER, average degree 3 & $66 \%$ & $100 \%$ & $100 \%$ & $26 \%$ & $74 \%$ & $100 \%$ \\
\hline & homogeneous & ER, average degree 6 & $100 \%$ & $100 \%$ & $100 \%$ & $100 \%$ & $100 \%$ & $100 \%$ \\
\hline & & SF, average degree 3 & $29 \%$ & $72 \%$ & $100 \%$ & $19 \%$ & $53 \%$ & $88 \%$ \\
\hline & & SF, average degree 6 & $100 \%$ & $100 \%$ & $100 \%$ & $88 \%$ & $100 \%$ & $100 \%$ \\
\hline & & in-arborescence & $0 \%$ & $1 \%$ & $12 \%$ & $0 \%$ & $1 \%$ & $11 \%$ \\
\hline & & ER, average degree 3 & $0 \%$ & $0 \%$ & $15 \%$ & $0 \%$ & $1 \%$ & $10 \%$ \\
\hline$|V|=100$ & $(0.1,0.95)$-heterogeneous & ER, average degree 6 & $6 \%$ & $7 \%$ & $16 \%$ & $6 \%$ & $6 \%$ & $10 \%$ \\
\hline & & SF, average degree 3 & $0 \%$ & $6 \%$ & $23 \%$ & $0 \%$ & $0 \%$ & $16 \%$ \\
\hline & & SF, average degree 6 & $8 \%$ & $16 \%$ & $30 \%$ & $6 \%$ & $12 \%$ & $19 \%$ \\
\hline & & in-arborescence & $0 \%$ & $1 \%$ & $11 \%$ & $0 \%$ & $1 \%$ & $11 \%$ \\
\hline & & ER, average degree 3 & $6 \%$ & $10 \%$ & $18 \%$ & $0 \%$ & $7 \%$ & $16 \%$ \\
\hline & $(0.2,0.6)$-heterogeneous & ER, average degree 6 & $7 \%$ & $12 \%$ & $22 \%$ & $6 \%$ & $8 \%$ & $17 \%$ \\
\hline & & SF, average degree 3 & $0 \%$ & $2 \%$ & $18 \%$ & $0 \%$ & $0 \%$ & $16 \%$ \\
\hline & & SF, average degree 6 & $5 \%$ & $9 \%$ & $18 \%$ & $2 \%$ & $6 \%$ & $16 \%$ \\
\hline & & in-arborescence & $76 \%$ & 78\% & $81 \%$ & $36 \%$ & $67 \%$ & $81 \%$ \\
\hline & & ER, average degree 3 & $76 \%$ & $100 \%$ & $100 \%$ & $22 \%$ & $73 \%$ & $93 \%$ \\
\hline & homogeneous & ER, average degree 6 & $100 \%$ & $100 \%$ & $100 \%$ & $100 \%$ & $100 \%$ & $100 \%$ \\
\hline & & SF, average degree 3 & $26 \%$ & $52 \%$ & $100 \%$ & $20 \%$ & $42 \%$ & $88 \%$ \\
\hline & & SF, average degree 6 & $100 \%$ & $100 \%$ & $100 \%$ & $87 \%$ & $100 \%$ & $100 \%$ \\
\hline & & in-arborescence & $0 \%$ & $1 \%$ & $12 \%$ & $0 \%$ & $1 \%$ & $12 \%$ \\
\hline & & ER, average degree 3 & $0 \%$ & $2 \%$ & $16 \%$ & $0 \%$ & $0 \%$ & $5 \%$ \\
\hline$|V|=300$ & $(0.1,0.95)$-heterogeneous & ER, average degree 6 & $0 \%$ & $6 \%$ & $12 \%$ & $0 \%$ & $2 \%$ & $6 \%$ \\
\hline & & SF, average degree 3 & $0 \%$ & $9 \%$ & $24 \%$ & $0 \%$ & $1 \%$ & $16 \%$ \\
\hline & & SF, average degree 6 & $7 \%$ & $16 \%$ & $28 \%$ & $6 \%$ & $9 \%$ & $19 \%$ \\
\hline & & in-arborescence & $0 \%$ & $1 \%$ & $11 \%$ & $0 \%$ & $1 \%$ & $11 \%$ \\
\hline & & ER, average degree 3 & $6 \%$ & $8 \%$ & $16 \%$ & $0 \%$ & $2 \%$ & $16 \%$ \\
\hline & $(0.2,0.6)$-heterogeneous & ER, average degree 6 & $6 \%$ & $8 \%$ & $17 \%$ & $6 \%$ & $7 \%$ & $16 \%$ \\
\hline & & SF, average degree 3 & $0 \%$ & $1 \%$ & $16 \%$ & $0 \%$ & $0 \%$ & $16 \%$ \\
\hline & & SF, average degree 6 & $2 \%$ & $6 \%$ & $16 \%$ & $2 \%$ & $5 \%$ & $16 \%$ \\
\hline
\end{tabular}


STABILITY OF FINANCIAL NETWORKS

Table S2. Residual instabilities of homogeneous versus heterogeneous networks under coordinated shocks. The percentages shown are the percentages of networks for which $\xi<0.05$ or $\xi<0.1$ or $\xi<0.2$.

\begin{tabular}{|c|c|c|c|c|c|c|c|c|}
\hline & & & \multicolumn{6}{|c|}{ coordinated shock } \\
\hline & & & \multicolumn{3}{|c|}{$\Phi=0.6, \gamma=0.55$} & \multicolumn{3}{|c|}{$\Phi=0.6, \gamma=0.50$} \\
\hline & & & $\xi<0.05$ & $\xi<0.1$ & $\xi<0.2$ & $\xi<0.05$ & $\xi<0.1$ & $\xi<0.2$ \\
\hline \multirow{15}{*}{$|V|=50$} & \multirow{5}{*}{ homogeneous } & in-arborescence & $93 \%$ & $93 \%$ & $93 \%$ & $2 \%$ & $69 \%$ & $93 \%$ \\
\hline & & ER, average degree 3 & $97 \%$ & $100 \%$ & $100 \%$ & $64 \%$ & $95 \%$ & $100 \%$ \\
\hline & & ER, average degree 6 & $100 \%$ & $100 \%$ & $100 \%$ & $100 \%$ & $100 \%$ & $100 \%$ \\
\hline & & $\mathrm{SF}$, average degree 3 & $56 \%$ & $96 \%$ & $100 \%$ & $44 \%$ & $87 \%$ & $100 \%$ \\
\hline & & SF, average degree 6 & $100 \%$ & $100 \%$ & $100 \%$ & $100 \%$ & $100 \%$ & $100 \%$ \\
\hline & \multirow{5}{*}{$(0.1,0.95)$-heterogeneous } & in-arborescence & $0 \%$ & $0 \%$ & $1 \%$ & $0 \%$ & $0 \%$ & $0 \%$ \\
\hline & & ER, average degree 3 & $0 \%$ & $0 \%$ & $5 \%$ & $0 \%$ & $0 \%$ & $1 \%$ \\
\hline & & ER, average degree 6 & $9 \%$ & $11 \%$ & $14 \%$ & $5 \%$ & $7 \%$ & $7 \%$ \\
\hline & & SF, average degree 3 & $5 \%$ & $9 \%$ & $19 \%$ & $0 \%$ & $3 \%$ & $8 \%$ \\
\hline & & SF, average degree 6 & $22 \%$ & $28 \%$ & $41 \%$ & $10 \%$ & $15 \%$ & $17 \%$ \\
\hline & \multirow{5}{*}{$(0.2,0.6)$-heterogeneous } & in-arborescence & $0 \%$ & $0 \%$ & $9 \%$ & $0 \%$ & $0 \%$ & $9 \%$ \\
\hline & & ER, average degree 3 & $5 \%$ & $7 \%$ & $20 \%$ & $3 \%$ & $7 \%$ & $16 \%$ \\
\hline & & ER, average degree 6 & $11 \%$ & $14 \%$ & $26 \%$ & $7 \%$ & $7 \%$ & $19 \%$ \\
\hline & & SF, average degree 3 & $2 \%$ & $8 \%$ & $23 \%$ & $1 \%$ & $3 \%$ & $18 \%$ \\
\hline & & SF, average degree 6 & $9 \%$ & $15 \%$ & $26 \%$ & $7 \%$ & $8 \%$ & $18 \%$ \\
\hline \multirow{15}{*}{$|V|=100$} & \multirow{5}{*}{ homogeneous } & in-arborescence & 93\% & $93 \%$ & $93 \%$ & $23 \%$ & $84 \%$ & $93 \%$ \\
\hline & & ER, average degree 3 & $85 \%$ & $100 \%$ & $100 \%$ & $47 \%$ & $86 \%$ & $100 \%$ \\
\hline & & ER, average degree 6 & $100 \%$ & $100 \%$ & $100 \%$ & $100 \%$ & $100 \%$ & $100 \%$ \\
\hline & & SF, average degree 3 & $37 \%$ & $75 \%$ & $100 \%$ & $35 \%$ & $71 \%$ & $100 \%$ \\
\hline & & SF, average degree 6 & $100 \%$ & $100 \%$ & $100 \%$ & $96 \%$ & $100 \%$ & $100 \%$ \\
\hline & \multirow{5}{*}{$(0.1,0.95)$-heterogeneous } & in-arborescence & $0 \%$ & $0 \%$ & $0 \%$ & $0 \%$ & $0 \%$ & $0 \%$ \\
\hline & & ER, average degree 3 & $0 \%$ & $1 \%$ & $7 \%$ & $0 \%$ & $0 \%$ & $3 \%$ \\
\hline & & ER, average degree 6 & $7 \%$ & $7 \%$ & $9 \%$ & $7 \%$ & $7 \%$ & $7 \%$ \\
\hline & & SF, average degree 3 & $0 \%$ & $1 \%$ & $9 \%$ & $0 \%$ & $0 \%$ & $5 \%$ \\
\hline & & SF, average degree 6 & $10 \%$ & $15 \%$ & $21 \%$ & $7 \%$ & $7 \%$ & $11 \%$ \\
\hline & \multirow{5}{*}{$(0.2,0.6)$-heterogeneous } & in-arborescence & $0 \%$ & $0 \%$ & $9 \%$ & $0 \%$ & $0 \%$ & $9 \%$ \\
\hline & & ER, average degree 3 & $0 \%$ & $7 \%$ & $17 \%$ & $0 \%$ & $5 \%$ & $16 \%$ \\
\hline & & ER, average degree 6 & $7 \%$ & $7 \%$ & $19 \%$ & $7 \%$ & $7 \%$ & $16 \%$ \\
\hline & & SF, average degree 3 & $1 \%$ & $3 \%$ & $15 \%$ & $0 \%$ & $1 \%$ & $13 \%$ \\
\hline & & SF, average degree 6 & $7 \%$ & $9 \%$ & $18 \%$ & $7 \%$ & $7 \%$ & $17 \%$ \\
\hline \multirow{15}{*}{$|V|=300$} & \multirow{10}{*}{$(0.1,0.95)$-heterogeneous } & in-arborescence & $93 \%$ & $93 \%$ & $93 \%$ & $79 \%$ & $93 \%$ & $93 \%$ \\
\hline & & ER, average degree 3 & $93 \%$ & $100 \%$ & $100 \%$ & $48 \%$ & $85 \%$ & $100 \%$ \\
\hline & & ER, average degree 6 & $100 \%$ & $100 \%$ & $100 \%$ & $100 \%$ & $100 \%$ & $100 \%$ \\
\hline & & SF, average degree 3 & $28 \%$ & $56 \%$ & $97 \%$ & $28 \%$ & $56 \%$ & $97 \%$ \\
\hline & & SF, average degree 6 & $100 \%$ & $100 \%$ & $100 \%$ & $93 \%$ & $100 \%$ & $100 \%$ \\
\hline & & in-arborescence & $0 \%$ & $0 \%$ & $0 \%$ & $0 \%$ & $0 \%$ & $0 \%$ \\
\hline & & ER, average degree 3 & $0 \%$ & $1 \%$ & $7 \%$ & $0 \%$ & $0 \%$ & $3 \%$ \\
\hline & & ER, average degree 6 & $7 \%$ & $7 \%$ & $7 \%$ & $3 \%$ & $7 \%$ & $7 \%$ \\
\hline & & SF, average degree 3 & $0 \%$ & $0 \%$ & $11 \%$ & $0 \%$ & $0 \%$ & $3 \%$ \\
\hline & & SF, average degree 6 & $7 \%$ & $7 \%$ & $18 \%$ & $7 \%$ & $7 \%$ & $7 \%$ \\
\hline & \multirow{5}{*}{$(0.2,0.6)$-heterogeneous } & in-arborescence & $0 \%$ & $0 \%$ & $9 \%$ & $0 \%$ & $0 \%$ & $9 \%$ \\
\hline & & ER, average degree 3 & $1 \%$ & $7 \%$ & $17 \%$ & $1 \%$ & $5 \%$ & $16 \%$ \\
\hline & & ER, average degree 6 & $7 \%$ & $7 \%$ & $16 \%$ & $7 \%$ & $7 \%$ & $16 \%$ \\
\hline & & SF, average degree 3 & $0 \%$ & $1 \%$ & $13 \%$ & $0 \%$ & $0 \%$ & $13 \%$ \\
\hline & & SF, average degree 6 & $7 \%$ & $7 \%$ & $16 \%$ & $7 \%$ & $7 \%$ & $16 \%$ \\
\hline
\end{tabular}


DASGUPTA AND KALIGOUNDER

Table S3. Residual instabilities of homogeneous versus heterogeneous networks under coordinated shocks. The percentages shown are the percentages of networks for which $\xi<0.05$ or $\xi<0.1$ or $\xi<0.2$.

\begin{tabular}{|c|c|c|c|c|c|c|c|c|}
\hline & & & & & coordine & ed shock & & \\
\hline & & & $\Phi=$ & $0.7, \gamma=0$ & 65 & $\Phi=$ & $0.7, \gamma=0$ & \\
\hline & & & $\xi<0.05$ & $\xi<0.1$ & $\xi<0.2$ & $\xi<0.05$ & $\xi<0.1$ & $\xi<0.2$ \\
\hline & & in-arborescence & $93 \%$ & $93 \%$ & $93 \%$ & $35 \%$ & $\mathbf{8 8 \%}$ & $93 \%$ \\
\hline & & ER, average degree 3 & $97 \%$ & $100 \%$ & $100 \%$ & $81 \%$ & $100 \%$ & $100 \%$ \\
\hline & homogeneous & ER, average degree 6 & $100 \%$ & $100 \%$ & $100 \%$ & $100 \%$ & $100 \%$ & $100 \%$ \\
\hline & & SF, average degree 3 & $56 \%$ & $96 \%$ & $100 \%$ & $52 \%$ & $96 \%$ & $100 \%$ \\
\hline & & SF, average degree 6 & $100 \%$ & $100 \%$ & $100 \%$ & $100 \%$ & $100 \%$ & $100 \%$ \\
\hline & & in-arborescence & $0 \%$ & $1 \%$ & $7 \%$ & $0 \%$ & $0 \%$ & $0 \%$ \\
\hline & & ER, average degree 3 & $0 \%$ & $0 \%$ & $5 \%$ & $0 \%$ & $0 \%$ & $1 \%$ \\
\hline$|V|=50$ & $(0.1,0.95)$-heterogeneous & ER, average degree 6 & $10 \%$ & $13 \%$ & $16 \%$ & $7 \%$ & $7 \%$ & $9 \%$ \\
\hline & & SF, average degree 3 & $7 \%$ & $12 \%$ & $25 \%$ & $1 \%$ & $4 \%$ & $9 \%$ \\
\hline & & SF, average degree 6 & $26 \%$ & $32 \%$ & $49 \%$ & $14 \%$ & $15 \%$ & $21 \%$ \\
\hline & & in-arborescence & $0 \%$ & $0 \%$ & $10 \%$ & $0 \%$ & $0 \%$ & $9 \%$ \\
\hline & & ER, average degree 3 & $5 \%$ & $8 \%$ & $21 \%$ & $3 \%$ & $7 \%$ & $17 \%$ \\
\hline & $(0.2,0.6)$-heterogeneous & ER, average degree 6 & $13 \%$ & $18 \%$ & $30 \%$ & $7 \%$ & $7 \%$ & $20 \%$ \\
\hline & & SF, average degree 3 & $3 \%$ & $9 \%$ & $25 \%$ & $1 \%$ & $3 \%$ & $19 \%$ \\
\hline & & SF, average degree 6 & $9 \%$ & $16 \%$ & $28 \%$ & $8 \%$ & $9 \%$ & $19 \%$ \\
\hline & & in-arborescence & $93 \%$ & $93 \%$ & $93 \%$ & $31 \%$ & $93 \%$ & $93 \%$ \\
\hline & & ER, average degree 3 & $85 \%$ & $100 \%$ & $100 \%$ & $62 \%$ & $97 \%$ & $100 \%$ \\
\hline & homogeneous & ER, average degree 6 & $100 \%$ & $100 \%$ & $100 \%$ & $100 \%$ & $100 \%$ & $100 \%$ \\
\hline & & SF, average degree 3 & $37 \%$ & $75 \%$ & $100 \%$ & $37 \%$ & $75 \%$ & $100 \%$ \\
\hline & & SF, average degree 6 & $100 \%$ & $100 \%$ & $100 \%$ & $100 \%$ & $100 \%$ & $100 \%$ \\
\hline & & in-arborescence & $0 \%$ & $0 \%$ & $0 \%$ & $0 \%$ & $0 \%$ & $0 \%$ \\
\hline & & ER, average degree 3 & $0 \%$ & $1 \%$ & $7 \%$ & $0 \%$ & $0 \%$ & $5 \%$ \\
\hline$|V|=100$ & $(0.1,0.95)$-heterogeneous & ER, average degree 6 & $7 \%$ & $7 \%$ & $9 \%$ & $7 \%$ & $7 \%$ & $7 \%$ \\
\hline & & SF, average degree 3 & $0 \%$ & $2 \%$ & $15 \%$ & $0 \%$ & $0 \%$ & $5 \%$ \\
\hline & & SF, average degree 6 & $11 \%$ & $15 \%$ & $27 \%$ & $7 \%$ & $7 \%$ & $11 \%$ \\
\hline & & in-arborescence & $0 \%$ & $0 \%$ & $9 \%$ & $0 \%$ & $0 \%$ & $9 \%$ \\
\hline & & ER, average degree 3 & $0 \%$ & $7 \%$ & $18 \%$ & $0 \%$ & $7 \%$ & $16 \%$ \\
\hline & $(0.2,0.6)$-heterogeneous & ER, average degree 6 & $7 \%$ & $7 \%$ & $19 \%$ & $7 \%$ & $7 \%$ & $16 \%$ \\
\hline & & SF, average degree 3 & $1 \%$ & $4 \%$ & $17 \%$ & $0 \%$ & $2 \%$ & $13 \%$ \\
\hline & & SF, average degree 6 & $8 \%$ & $10 \%$ & $19 \%$ & $7 \%$ & $8 \%$ & $17 \%$ \\
\hline & & in-arborescence & 93\% & $93 \%$ & $93 \%$ & $56 \%$ & $93 \%$ & $93 \%$ \\
\hline & & ER, average degree 3 & $93 \%$ & $100 \%$ & $100 \%$ & $65 \%$ & $97 \%$ & $100 \%$ \\
\hline & homogeneous & ER, average degree 6 & $100 \%$ & $100 \%$ & $100 \%$ & $100 \%$ & $100 \%$ & $100 \%$ \\
\hline & & SF, average degree 3 & $28 \%$ & $56 \%$ & $97 \%$ & $28 \%$ & $56 \%$ & $97 \%$ \\
\hline & & SF, average degree 6 & $100 \%$ & $100 \%$ & $100 \%$ & $100 \%$ & $100 \%$ & $100 \%$ \\
\hline & & in-arborescence & $0 \%$ & $0 \%$ & $0 \%$ & $0 \%$ & $0 \%$ & $0 \%$ \\
\hline & & ER, average degree 3 & $0 \%$ & $1 \%$ & $7 \%$ & $0 \%$ & $0 \%$ & $4 \%$ \\
\hline$|V|=300$ & $(0.1,0.95)$-heterogeneous & ER, average degree 6 & $7 \%$ & $7 \%$ & $7 \%$ & $6 \%$ & $7 \%$ & $7 \%$ \\
\hline & & SF, average degree 3 & $0 \%$ & $0 \%$ & $15 \%$ & $0 \%$ & $0 \%$ & $3 \%$ \\
\hline & & SF, average degree 6 & $7 \%$ & $7 \%$ & $21 \%$ & $7 \%$ & $7 \%$ & $7 \%$ \\
\hline & & in-arborescence & $0 \%$ & $0 \%$ & $9 \%$ & $0 \%$ & $0 \%$ & $9 \%$ \\
\hline & & ER, average degree 3 & $1 \%$ & $7 \%$ & $17 \%$ & $0 \%$ & $7 \%$ & $16 \%$ \\
\hline & $(0.2,0.6)$-heterogeneous & ER, average degree 6 & $7 \%$ & $7 \%$ & $16 \%$ & $7 \%$ & $7 \%$ & $16 \%$ \\
\hline & & SF, average degree 3 & $0 \%$ & $1 \%$ & $14 \%$ & $0 \%$ & $0 \%$ & $13 \%$ \\
\hline & & SF, average degree 6 & $7 \%$ & $8 \%$ & $16 \%$ & $7 \%$ & $7 \%$ & $16 \%$ \\
\hline
\end{tabular}


STABILITY OF FINANCIAL NETWORKS

Table S4. Residual instabilities of homogeneous versus heterogeneous networks under coordinated shocks. The percentages shown are the percentages of networks for which $\xi<0.05$ or $\xi<0.1$ or $\xi<0.2$.

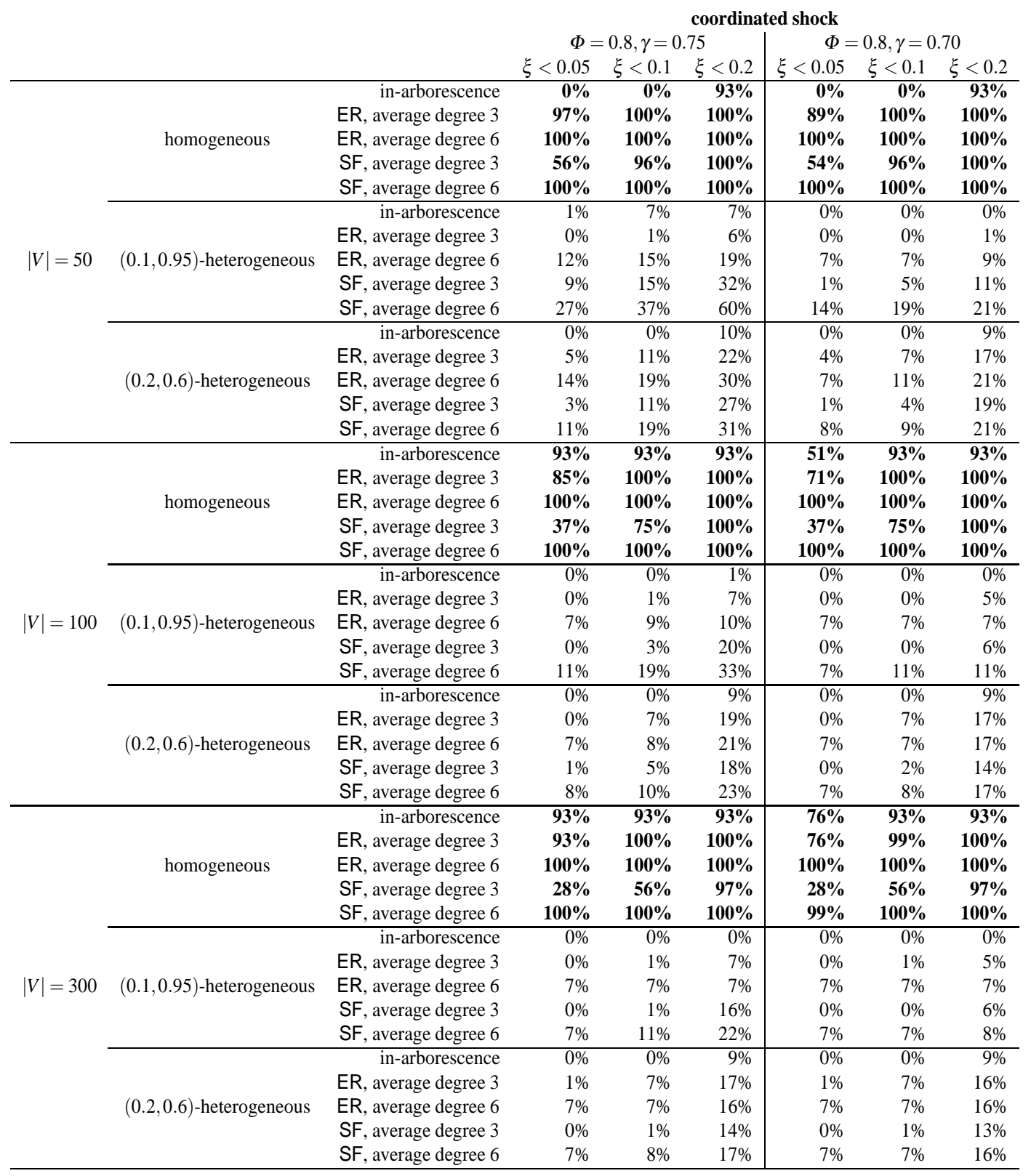




\section{DASGUPTA AND KALIGOUNDER}

Table S5. Residual instabilities of homogeneous versus heterogeneous networks under coordinated shocks. The percentages shown are the percentages of networks for which $\xi<0.05$ or $\xi<0.1$ or $\xi<0.2$.

\begin{tabular}{|c|c|c|c|c|c|c|c|c|}
\hline & & & & & coordine & ed shock & & \\
\hline & & & $\Phi=$ & $0.9, \gamma=0$ & 85 & $\Phi=$ & $0.9, \gamma=0$ & \\
\hline & & & $\xi<0.05$ & $\xi<0.1$ & $\xi<0.2$ & $\xi<0.05$ & $\xi<0.1$ & $\xi<0.2$ \\
\hline & & in-arborescence & $93 \%$ & $93 \%$ & $93 \%$ & $81 \%$ & $93 \%$ & $93 \%$ \\
\hline & & ER, average degree 3 & $98 \%$ & $100 \%$ & $100 \%$ & $95 \%$ & $100 \%$ & $100 \%$ \\
\hline & homogeneous & ER, average degree 6 & $0 \%$ & $0 \%$ & $0 \%$ & $100 \%$ & $100 \%$ & $100 \%$ \\
\hline & & SF, average degree 3 & $56 \%$ & $96 \%$ & $100 \%$ & $56 \%$ & $96 \%$ & $100 \%$ \\
\hline & & SF, average degree 6 & $100 \%$ & $100 \%$ & $100 \%$ & $100 \%$ & $100 \%$ & $100 \%$ \\
\hline & & in-arborescence & $7 \%$ & $7 \%$ & $7 \%$ & $0 \%$ & $0 \%$ & $0 \%$ \\
\hline & & ER, average degree 3 & $0 \%$ & $1 \%$ & $7 \%$ & $0 \%$ & $0 \%$ & $1 \%$ \\
\hline$|V|=50$ & $(0.1,0.95)$-heterogeneous & ER, average degree 6 & $13 \%$ & $18 \%$ & $22 \%$ & $7 \%$ & $9 \%$ & $10 \%$ \\
\hline & & SF, average degree 3 & $11 \%$ & $17 \%$ & $37 \%$ & $3 \%$ & $5 \%$ & $13 \%$ \\
\hline & & SF, average degree 6 & $31 \%$ & $41 \%$ & $66 \%$ & $18 \%$ & $19 \%$ & $25 \%$ \\
\hline & & in-arborescence & $0 \%$ & $0 \%$ & $15 \%$ & $0 \%$ & $0 \%$ & $9 \%$ \\
\hline & & ER, average degree 3 & $5 \%$ & $11 \%$ & $25 \%$ & $5 \%$ & $7 \%$ & $19 \%$ \\
\hline & $(0.2,0.6)$-heterogeneous & ER, average degree 6 & $14 \%$ & $19 \%$ & $34 \%$ & $7 \%$ & $12 \%$ & $23 \%$ \\
\hline & & SF, average degree 3 & $4 \%$ & $12 \%$ & $28 \%$ & $2 \%$ & $6 \%$ & $21 \%$ \\
\hline & & SF, average degree 6 & $17 \%$ & $21 \%$ & $33 \%$ & $8 \%$ & $11 \%$ & $23 \%$ \\
\hline & & in-arborescence & $93 \%$ & $93 \%$ & $93 \%$ & $75 \%$ & $93 \%$ & $93 \%$ \\
\hline & & ER, average degree 3 & $85 \%$ & $100 \%$ & $100 \%$ & $78 \%$ & $100 \%$ & $100 \%$ \\
\hline & homogeneous & ER, average degree 6 & $0 \%$ & $0 \%$ & $0 \%$ & $100 \%$ & $100 \%$ & $100 \%$ \\
\hline & & SF, average degree 3 & $37 \%$ & $75 \%$ & $100 \%$ & $37 \%$ & $75 \%$ & $100 \%$ \\
\hline & & SF, average degree 6 & $100 \%$ & $100 \%$ & $100 \%$ & $100 \%$ & $100 \%$ & $100 \%$ \\
\hline & & in-arborescence & $0 \%$ & $0 \%$ & $1 \%$ & $0 \%$ & $0 \%$ & $0 \%$ \\
\hline & & ER, average degree 3 & $0 \%$ & $1 \%$ & $7 \%$ & $0 \%$ & $0 \%$ & $6 \%$ \\
\hline$|V|=100$ & $(0.1,0.95)$-heterogeneous & ER, average degree 6 & $7 \%$ & $9 \%$ & $10 \%$ & $7 \%$ & $7 \%$ & $7 \%$ \\
\hline & & SF, average degree 3 & $1 \%$ & $4 \%$ & $25 \%$ & $0 \%$ & $1 \%$ & $7 \%$ \\
\hline & & SF, average degree 6 & $14 \%$ & $22 \%$ & $41 \%$ & $7 \%$ & $11 \%$ & $15 \%$ \\
\hline & & in-arborescence & $0 \%$ & $0 \%$ & $9 \%$ & $0 \%$ & $0 \%$ & $9 \%$ \\
\hline & & ER, average degree 3 & $0 \%$ & $7 \%$ & $21 \%$ & $0 \%$ & $7 \%$ & $17 \%$ \\
\hline & $(0.2,0.6)$-heterogeneous & ER, average degree 6 & $7 \%$ & $8 \%$ & $23 \%$ & $7 \%$ & $7 \%$ & $17 \%$ \\
\hline & & SF, average degree 3 & $1 \%$ & $5 \%$ & $19 \%$ & $1 \%$ & $3 \%$ & $15 \%$ \\
\hline & & SF, average degree 6 & $9 \%$ & $11 \%$ & $23 \%$ & $7 \%$ & $9 \%$ & $17 \%$ \\
\hline & & in-arborescence & 93\% & $93 \%$ & $93 \%$ & $93 \%$ & $93 \%$ & $93 \%$ \\
\hline & & ER, average degree 3 & $0 \%$ & $0 \%$ & $100 \%$ & $85 \%$ & $99 \%$ & $100 \%$ \\
\hline & homogeneous & ER, average degree 6 & $0 \%$ & $0 \%$ & $0 \%$ & $100 \%$ & $100 \%$ & $100 \%$ \\
\hline & & SF, average degree 3 & $28 \%$ & $56 \%$ & $97 \%$ & $28 \%$ & $56 \%$ & $97 \%$ \\
\hline & & SF, average degree 6 & $100 \%$ & $100 \%$ & $100 \%$ & $100 \%$ & $100 \%$ & $100 \%$ \\
\hline & & in-arborescence & $0 \%$ & $0 \%$ & $0 \%$ & $0 \%$ & $0 \%$ & $0 \%$ \\
\hline & & ER, average degree 3 & $0 \%$ & $1 \%$ & $7 \%$ & $0 \%$ & $1 \%$ & $6 \%$ \\
\hline$|V|=300$ & $(0.1,0.95)$-heterogeneous & ER, average degree 6 & $7 \%$ & $7 \%$ & $7 \%$ & $7 \%$ & $7 \%$ & $7 \%$ \\
\hline & & SF, average degree 3 & $0 \%$ & $1 \%$ & $17 \%$ & $0 \%$ & $0 \%$ & $10 \%$ \\
\hline & & SF, average degree 6 & $7 \%$ & $11 \%$ & $24 \%$ & $7 \%$ & $7 \%$ & $10 \%$ \\
\hline & & in-arborescence & $0 \%$ & $0 \%$ & $9 \%$ & $0 \%$ & $0 \%$ & $9 \%$ \\
\hline & & ER, average degree 3 & $1 \%$ & $7 \%$ & $17 \%$ & $1 \%$ & $7 \%$ & $16 \%$ \\
\hline & $(0.2,0.6)$-heterogeneous & ER, average degree 6 & $7 \%$ & $7 \%$ & $17 \%$ & $7 \%$ & $7 \%$ & $16 \%$ \\
\hline & & SF, average degree 3 & $0 \%$ & $1 \%$ & $14 \%$ & $0 \%$ & $1 \%$ & $13 \%$ \\
\hline & & SF, average degree 6 & $7 \%$ & $8 \%$ & $17 \%$ & $7 \%$ & $7 \%$ & $16 \%$ \\
\hline
\end{tabular}


STABILITY OF FINANCIAL NETWORKS

Table S6. Residual instabilities of homogeneous versus heterogeneous networks under coordinated shocks. The percentages shown are the percentages of networks for which $\xi<0.05$ or $\xi<0.1$ or $\xi<0.2$.

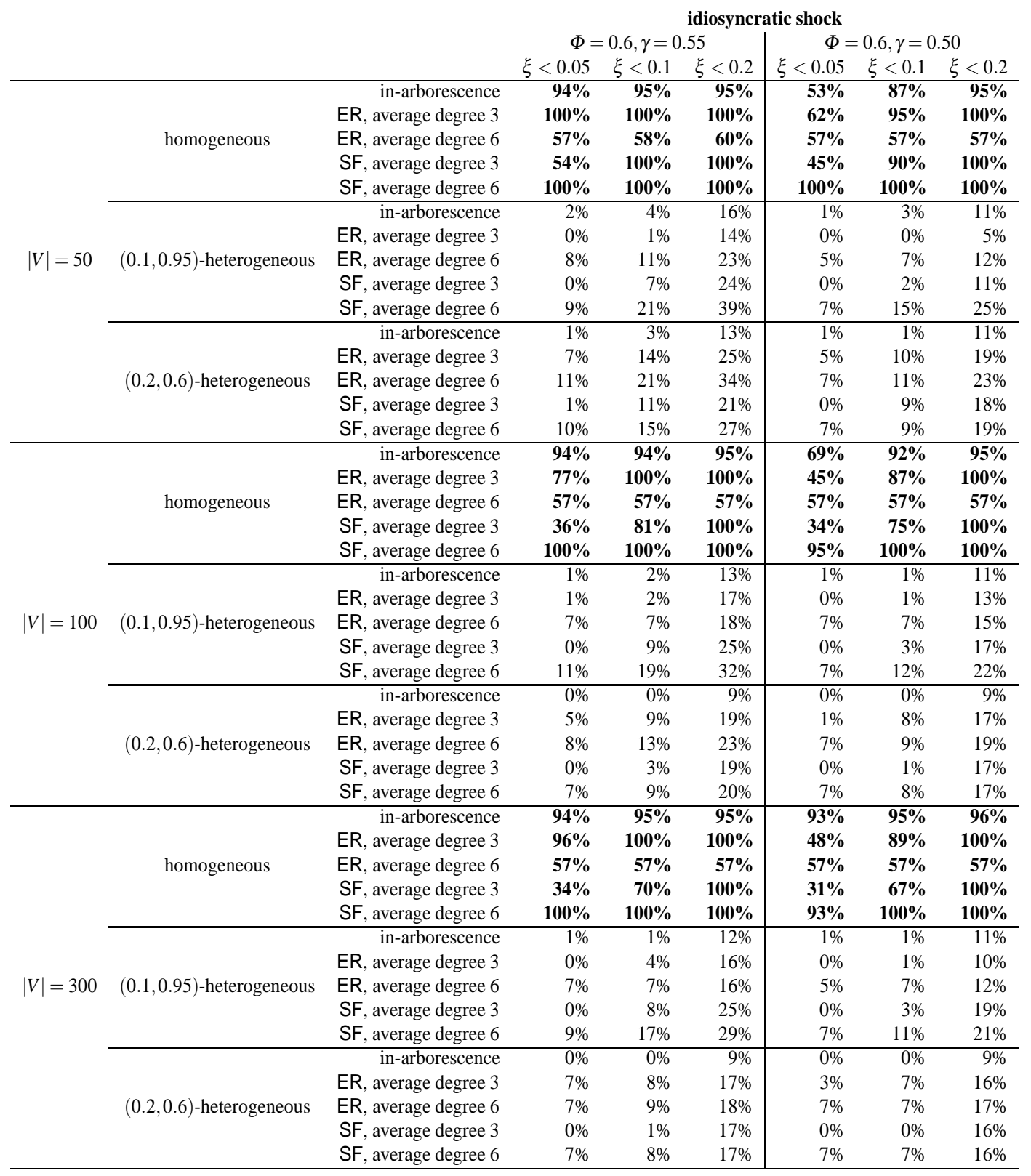


DASGUPTA AND KALIGOUNDER

Table S7. Residual instabilities of homogeneous versus heterogeneous networks under coordinated shocks. The percentages shown are the percentages of networks for which $\xi<0.05$ or $\xi<0.1$ or $\xi<0.2$.

\begin{tabular}{|c|c|c|c|c|c|c|c|c|}
\hline & & & & & idiosyncr & tic shock & & \\
\hline & & & $\Phi=$ & $0.7, \gamma=0$ & & $\Phi=$ & $0.7, \gamma=0$ & \\
\hline & & & $\xi<0.05$ & $\xi<0.1$ & $\xi<0.2$ & $\xi<0.05$ & $\xi<0.1$ & $\xi<0.2$ \\
\hline & & in-arborescence & $94 \%$ & $95 \%$ & $96 \%$ & 73\% & $94 \%$ & $95 \%$ \\
\hline & & ER, average degree 3 & $100 \%$ & $100 \%$ & $100 \%$ & $81 \%$ & $100 \%$ & $100 \%$ \\
\hline & homogeneous & ER, average degree 6 & $57 \%$ & $59 \%$ & $60 \%$ & $57 \%$ & $57 \%$ & $57 \%$ \\
\hline & & SF, average degree 3 & $55 \%$ & $100 \%$ & $100 \%$ & $55 \%$ & $100 \%$ & $100 \%$ \\
\hline & & SF, average degree 6 & $100 \%$ & $100 \%$ & $100 \%$ & $100 \%$ & $100 \%$ & $100 \%$ \\
\hline & & in-arborescence & $3 \%$ & $7 \%$ & $17 \%$ & $1 \%$ & $2 \%$ & $12 \%$ \\
\hline & & ER, average degree 3 & $1 \%$ & $5 \%$ & $21 \%$ & $0 \%$ & $3 \%$ & $13 \%$ \\
\hline$|V|=50$ & $(0.1,0.95)$-heterogeneous & ER, average degree 6 & $10 \%$ & $18 \%$ & $28 \%$ & $7 \%$ & $9 \%$ & $19 \%$ \\
\hline & & SF, average degree 3 & $7 \%$ & $9 \%$ & $26 \%$ & $0 \%$ & $3 \%$ & $19 \%$ \\
\hline & & SF, average degree 6 & $13 \%$ & $24 \%$ & $41 \%$ & $9 \%$ & $16 \%$ & $27 \%$ \\
\hline & & in-arborescence & $1 \%$ & $3 \%$ & $14 \%$ & $1 \%$ & $1 \%$ & $11 \%$ \\
\hline & & ER, average degree 3 & $7 \%$ & $15 \%$ & $27 \%$ & $7 \%$ & $10 \%$ & $20 \%$ \\
\hline & $(0.2,0.6)$-heterogeneous & ER, average degree 6 & $13 \%$ & $23 \%$ & $35 \%$ & $7 \%$ & $13 \%$ & $25 \%$ \\
\hline & & SF, average degree 3 & $1 \%$ & $11 \%$ & $23 \%$ & $0 \%$ & $6 \%$ & $19 \%$ \\
\hline & & SF, average degree 6 & $11 \%$ & $17 \%$ & $29 \%$ & $7 \%$ & $9 \%$ & $20 \%$ \\
\hline & & in-arborescence & $94 \%$ & $95 \%$ & $95 \%$ & $79 \%$ & $95 \%$ & $95 \%$ \\
\hline & & ER, average degree 3 & $77 \%$ & $100 \%$ & $100 \%$ & $57 \%$ & $97 \%$ & $100 \%$ \\
\hline & homogeneous & ER, average degree 6 & $57 \%$ & $57 \%$ & $\mathbf{5 7 \%}$ & $57 \%$ & $57 \%$ & $57 \%$ \\
\hline & & SF, average degree 3 & $37 \%$ & $78 \%$ & $100 \%$ & $35 \%$ & $76 \%$ & $100 \%$ \\
\hline & & SF, average degree 6 & $100 \%$ & $100 \%$ & $100 \%$ & $100 \%$ & $100 \%$ & $100 \%$ \\
\hline & & in-arborescence & $1 \%$ & $3 \%$ & $13 \%$ & $1 \%$ & $1 \%$ & $12 \%$ \\
\hline & & ER, average degree 3 & $0 \%$ & $4 \%$ & $17 \%$ & $0 \%$ & $1 \%$ & $14 \%$ \\
\hline$|V|=100$ & $(0.1,0.95)$-heterogeneous & ER, average degree 6 & $7 \%$ & $9 \%$ & $19 \%$ & $7 \%$ & $7 \%$ & $16 \%$ \\
\hline & & SF, average degree 3 & $0 \%$ & $10 \%$ & $25 \%$ & $0 \%$ & $3 \%$ & $19 \%$ \\
\hline & & SF, average degree 6 & $9 \%$ & $18 \%$ & $33 \%$ & $8 \%$ & $15 \%$ & $25 \%$ \\
\hline & & in-arborescence & $1 \%$ & $1 \%$ & $11 \%$ & $1 \%$ & $1 \%$ & $11 \%$ \\
\hline & & ER, average degree 3 & $6 \%$ & $11 \%$ & $21 \%$ & $3 \%$ & $9 \%$ & $17 \%$ \\
\hline & $(0.2,0.6)$-heterogeneous & ER, average degree 6 & $7 \%$ & $15 \%$ & $25 \%$ & $7 \%$ & $9 \%$ & $19 \%$ \\
\hline & & SF, average degree 3 & $0 \%$ & $3 \%$ & $19 \%$ & $0 \%$ & $1 \%$ & $17 \%$ \\
\hline & & SF, average degree 6 & $7 \%$ & $10 \%$ & $21 \%$ & $7 \%$ & $8 \%$ & $17 \%$ \\
\hline & & in-arborescence & $94 \%$ & $95 \%$ & $95 \%$ & $85 \%$ & $95 \%$ & $95 \%$ \\
\hline & & ER, average degree 3 & $96 \%$ & $100 \%$ & $100 \%$ & $67 \%$ & $98 \%$ & $100 \%$ \\
\hline & homogeneous & ER, average degree 6 & $57 \%$ & $57 \%$ & $57 \%$ & $57 \%$ & $\mathbf{5 7 \%}$ & $57 \%$ \\
\hline & & SF, average degree 3 & $33 \%$ & $71 \%$ & $100 \%$ & $33 \%$ & $69 \%$ & $100 \%$ \\
\hline & & SF, average degree 6 & $100 \%$ & $100 \%$ & $100 \%$ & $99 \%$ & $100 \%$ & $100 \%$ \\
\hline & & in-arborescence & $1 \%$ & $1 \%$ & $12 \%$ & $1 \%$ & $1 \%$ & $12 \%$ \\
\hline & & ER, average degree 3 & $1 \%$ & $5 \%$ & $16 \%$ & $0 \%$ & $2 \%$ & $13 \%$ \\
\hline$|V|=300$ & $(0.1,0.95)$-heterogeneous & ER, average degree 6 & $7 \%$ & $7 \%$ & $16 \%$ & $6 \%$ & $7 \%$ & $15 \%$ \\
\hline & & SF, average degree 3 & $0 \%$ & $9 \%$ & $25 \%$ & $0 \%$ & $3 \%$ & $19 \%$ \\
\hline & & SF, average degree 6 & $7 \%$ & $17 \%$ & $31 \%$ & $7 \%$ & $13 \%$ & $21 \%$ \\
\hline & & in-arborescence & $1 \%$ & $1 \%$ & $11 \%$ & $1 \%$ & $1 \%$ & $11 \%$ \\
\hline & & ER, average degree 3 & $7 \%$ & $8 \%$ & $17 \%$ & $3 \%$ & $7 \%$ & $17 \%$ \\
\hline & $(0.2,0.6)$-heterogeneous & ER, average degree 6 & $7 \%$ & $9 \%$ & $19 \%$ & $7 \%$ & $8 \%$ & $17 \%$ \\
\hline & & SF, average degree 3 & $0 \%$ & $3 \%$ & $17 \%$ & $0 \%$ & $1 \%$ & $16 \%$ \\
\hline & & SF, average degree 6 & $7 \%$ & $8 \%$ & $17 \%$ & $7 \%$ & $7 \%$ & $16 \%$ \\
\hline
\end{tabular}


STABILITY OF FINANCIAL NETWORKS

Table S8. Residual instabilities of homogeneous versus heterogeneous networks under coordinated shocks. The percentages shown are the percentages of networks for which $\xi<0.05$ or $\xi<0.1$ or $\xi<0.2$.

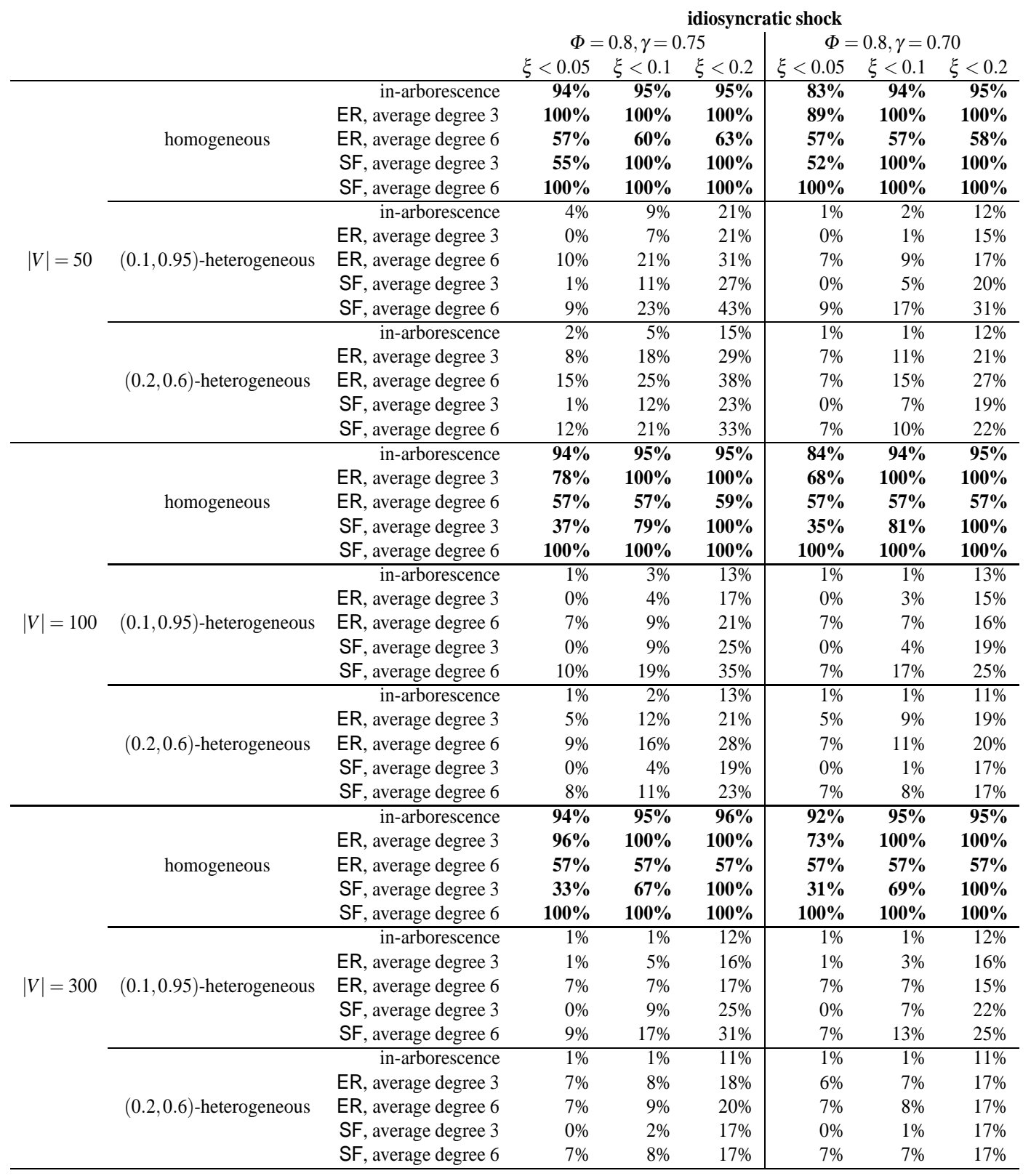




\section{DASGUPTA AND KALIGOUNDER}

Table S9. Residual instabilities of homogeneous versus heterogeneous networks under coordinated shocks. The percentages shown are the percentages of networks for which $\xi<0.05$ or $\xi<0.1$ or $\xi<0.2$.

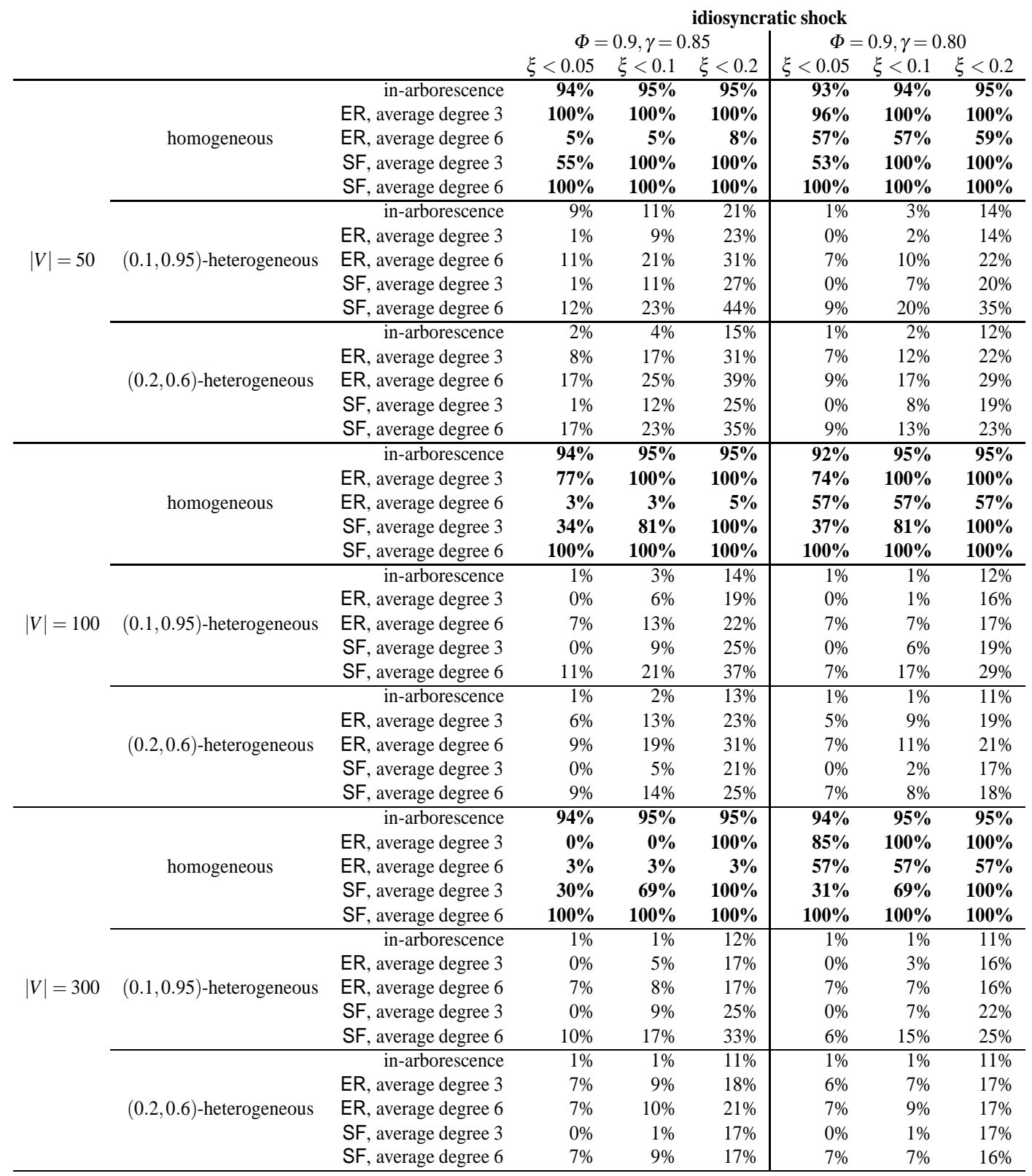


STABILITY OF FINANCIAL NETWORKS

\begin{abstract}
ER model (average degree 6), coordinated shock
ER model (average degree 3), coordinated shock

SF model (average degree 6), coordinated shock

SF model (average degree 3), coordinated shock
\end{abstract}

in-arborescence (average degree $\approx 1$ ), coordinated shock $\square=$

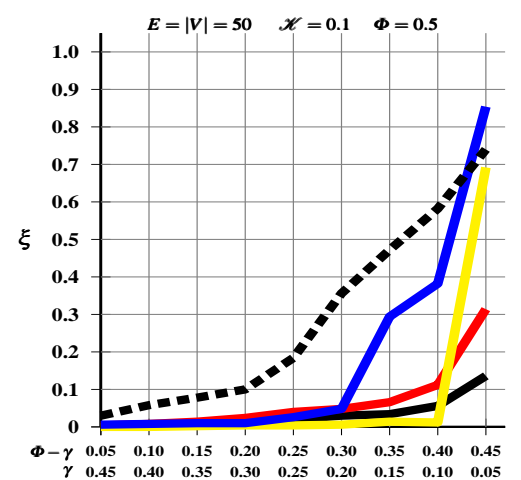

(A)

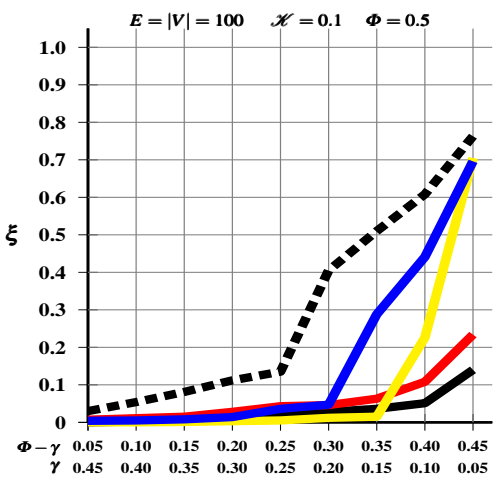

(D)

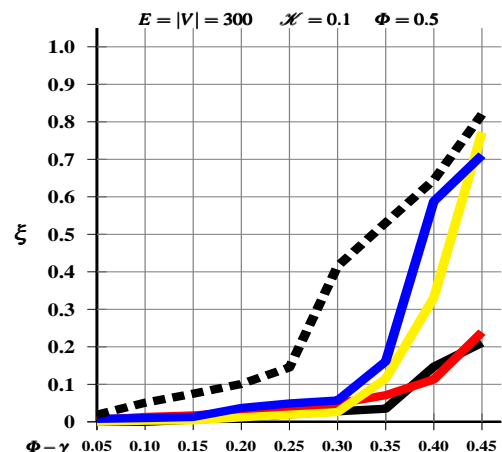

$\begin{array}{rrrrrrrrrr}\Phi-\gamma & 0.05 & 0.10 & 0.15 & 0.20 & 0.25 & 0.30 & 0.35 & 0.40 & 0.45 \\ \gamma & 0.45 & 0.40 & 0.35 & 0.30 & 0.25 & 0.20 & 0.15 & 0.10 & 0.05\end{array}$

(G)

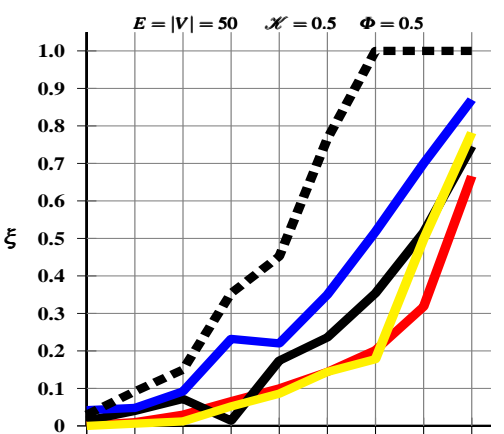

$\begin{array}{llllllllll}\Phi-\gamma & 0.05 & 0.10 & 0.15 & 0.20 & 0.25 & 0.30 & 0.35 & 0.40 & 0.45\end{array}$

$\begin{array}{llllllllll}\gamma & 0.05 & 0.10 & 0.15 & 0.20 & 0.25 & 0.30 & 0.35 & 0.40 & 0.45 \\ \gamma & 0.45 & 0.40 & 0.35 & 0.30 & 0.25 & 0.20 & 0.15 & 0.10 & 0.05\end{array}$

(B)

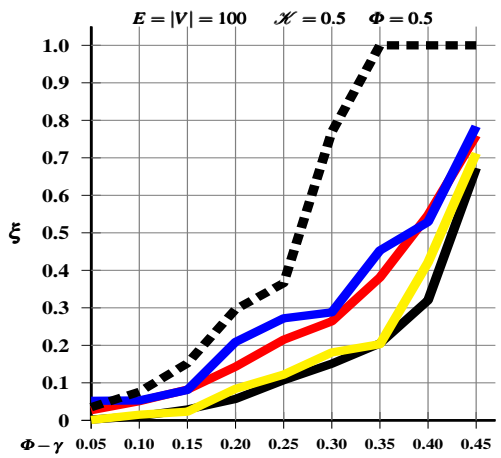

$\begin{array}{rrrrrrrrrr}\Phi-\gamma & 0.05 & 0.10 & 0.15 & 0.20 & 0.25 & 0.30 & 0.35 & 0.40 & 0.45 \\ \gamma & 0.45 & 0.40 & 0.35 & 0.30 & 0.25 & 0.20 & 0.15 & 0.10 & 0.05\end{array}$

(E)

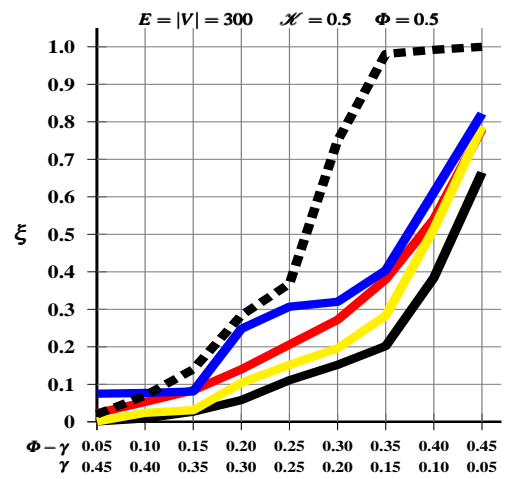

(H)

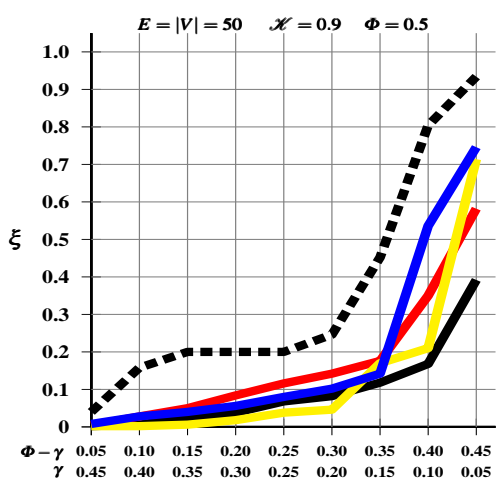

(C)

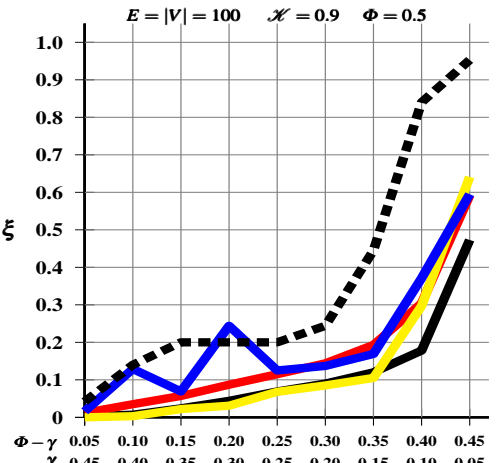

$\begin{array}{rlllllllll}\Phi-\gamma & 0.05 & 0.10 & 0.15 & 0.20 & 0.25 & 0.30 & 0.35 & 0.40 & 0.45 \\ \gamma & 0.45 & 0.40 & 0.35 & 0.30 & 0.25 & 0.20 & 0.15 & 0.10 & 0.05\end{array}$

(F)

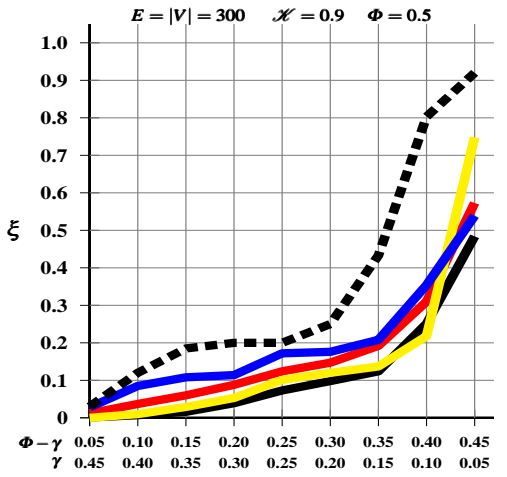

(I)

FIG. S1. Effect of variations of equity to asset ratio (with respect to shock) on the vulnerability index $\xi$ for homogeneous networks. Lower values of $\xi$ imply higher global stability of a network. 
ER model (average degree 6), coordinated shock

SF model (average degree 6), coordinated shock
ER model (average degree 3), coordinated shock

SF model (average degree 3 ), coordinated shock

in-arborescence (average degree $\approx 1$ ), coordinated shock $=\square$

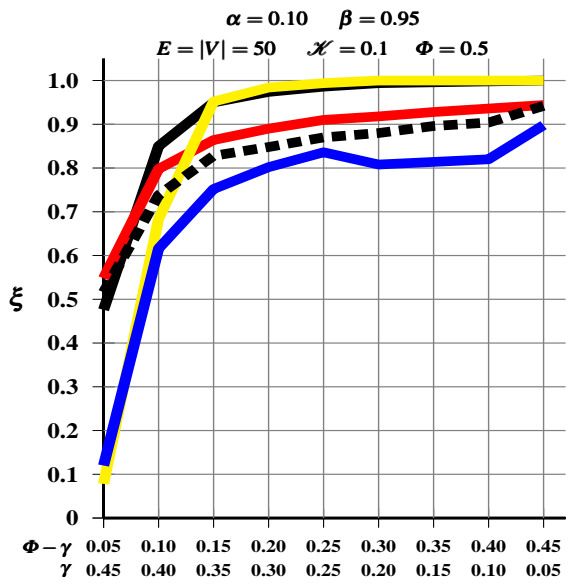

(A)

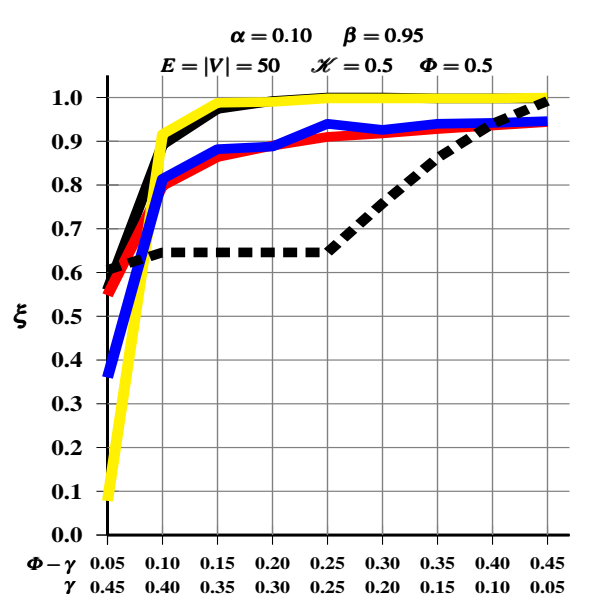

(B)

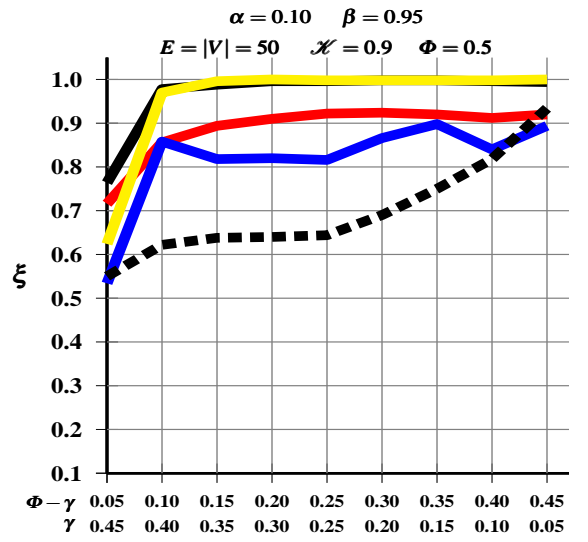

(C)

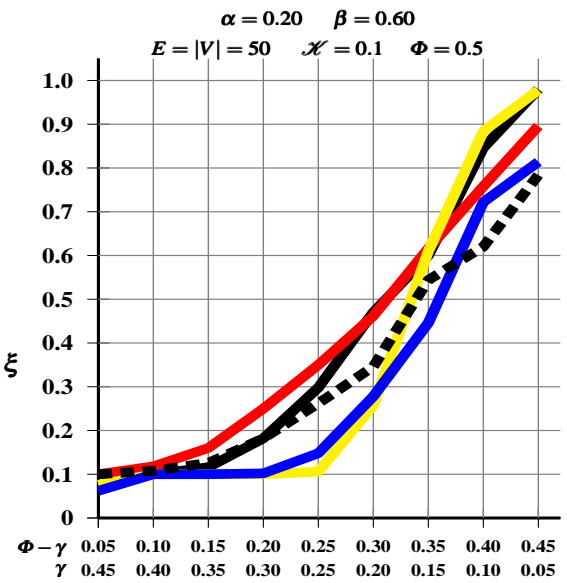

(D)

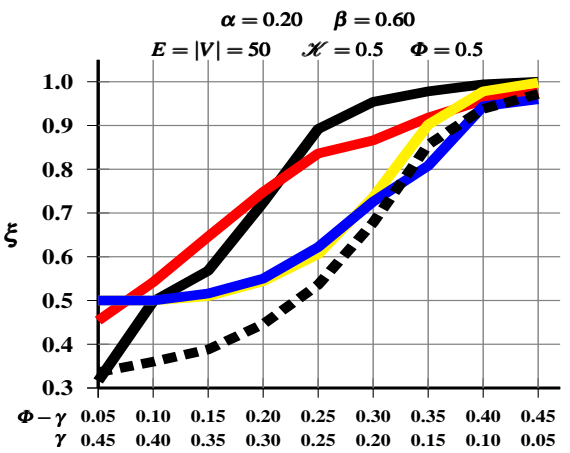

(E)

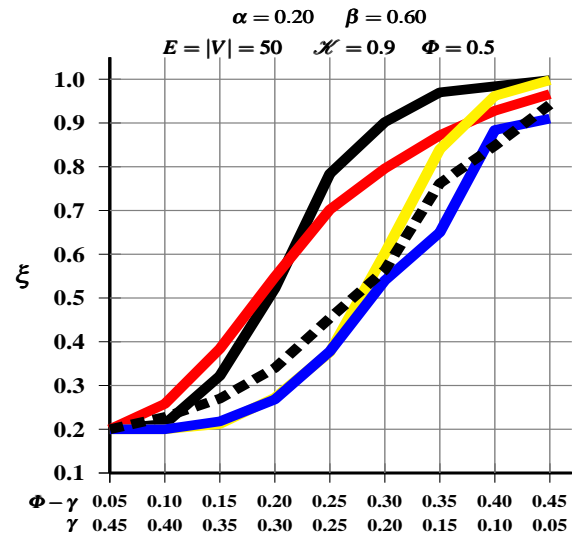

(F)

FIG. S2. Effect of variations of equity to asset ratio (with respect to shock) on the vulnerability index $\xi$ for $(\alpha, \beta)$-heterogeneous networks. Lower values of $\xi$ imply higher global stability of a network. 
STABILITY OF FINANCIAL NETWORKS

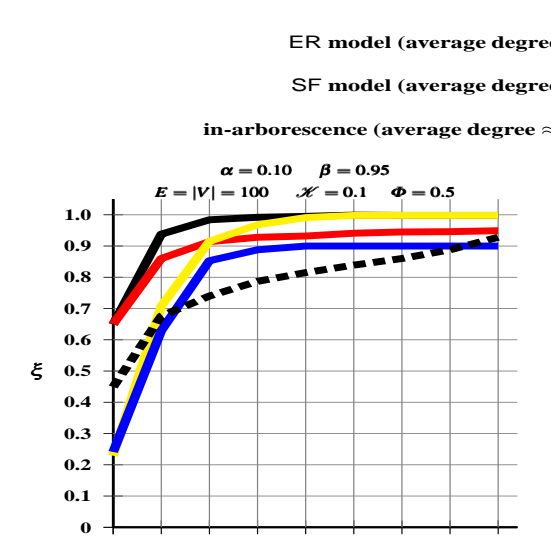

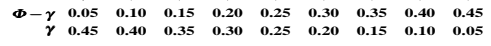
(A)

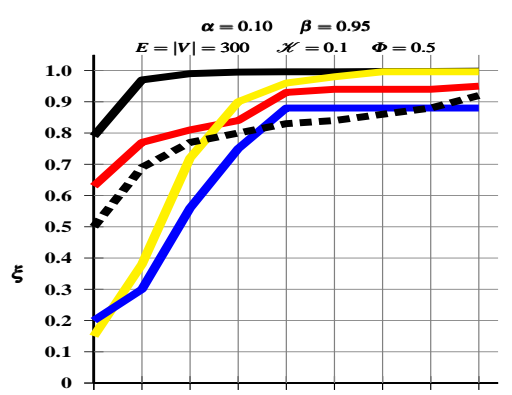

$\begin{array}{rlllllllll}\Phi-\gamma & 0.05 & 0.10 & 0.15 & 0.20 & 0.25 & 0.30 & 0.35 & 0.40 & 0.45 \\ \gamma & 0.45 & 0.40 & 0.35 & 0.30 & 0.25 & 0.20 & 0.15 & 0.10 & 0.05\end{array}$

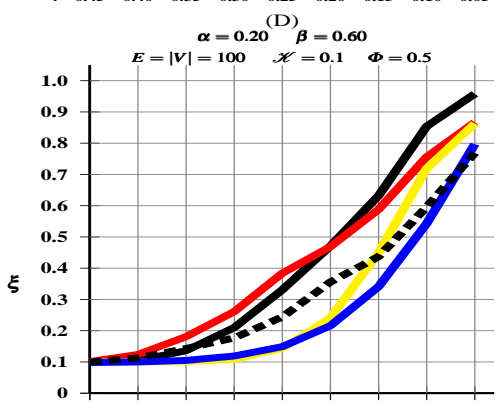

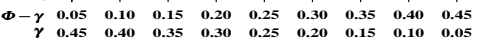
(G)

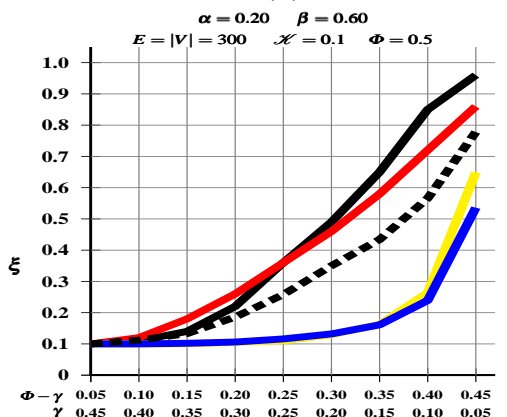

(J)
ER model (average degree 3), coordinated shock -

SF model (average degree 3), coordinated shock coordinated shock

oordinated shock $=\mathrm{m}$

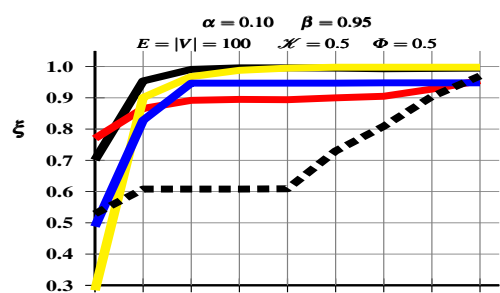

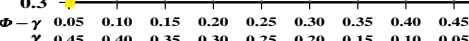

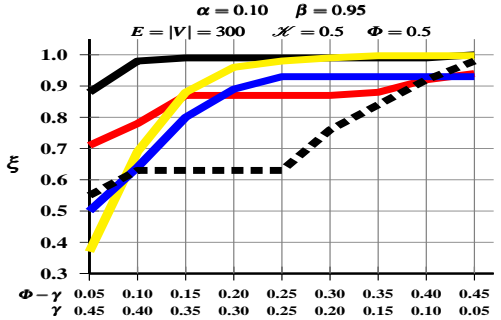

(E)

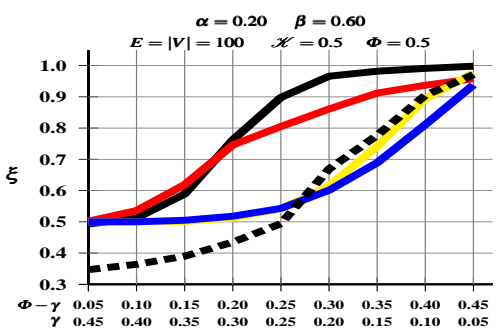

(H)

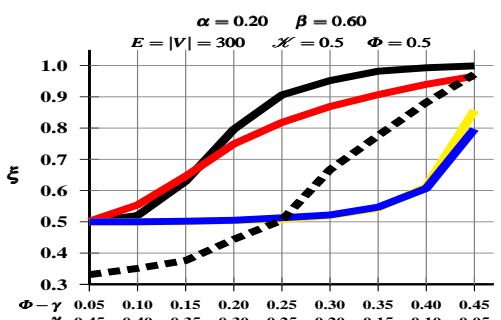

(K)

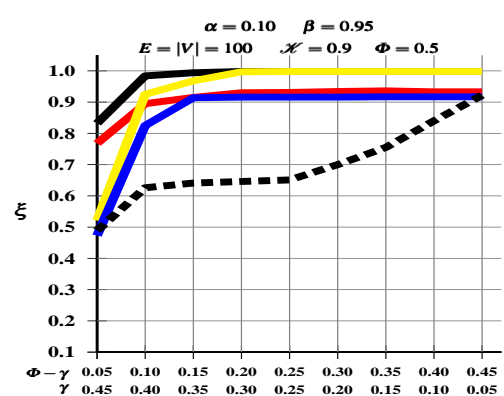

(C)

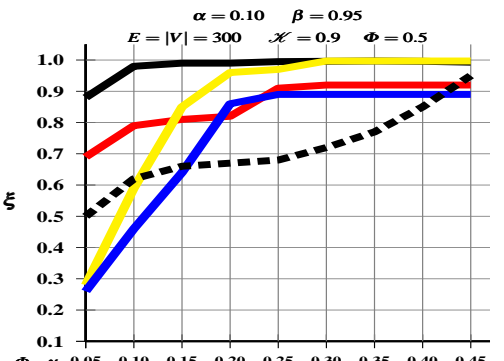

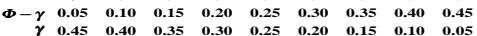

(F)

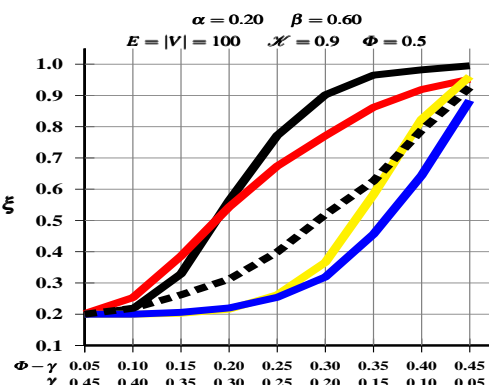

(I)

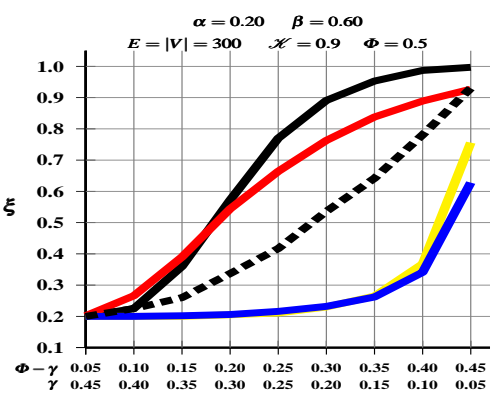

(L)

FIG. S3. Effect of variations of equity to asset ratio (with respect to shock) on the vulnerability index $\xi$ for $(\alpha, \beta)$-heterogeneous networks. Lower values of $\xi$ imply higher global stability of a network. 
DASGUPTA AND KALIGOUNDER

ER model (average degree 6), coordinated shock

SF model (average degree 6), coordinated shock
ER model (average degree 3), coordinated shock

SF model (average degree 3), coordinated shock

in-arborescence (average degree $\approx 1$ ), coordinated shock 1 u.

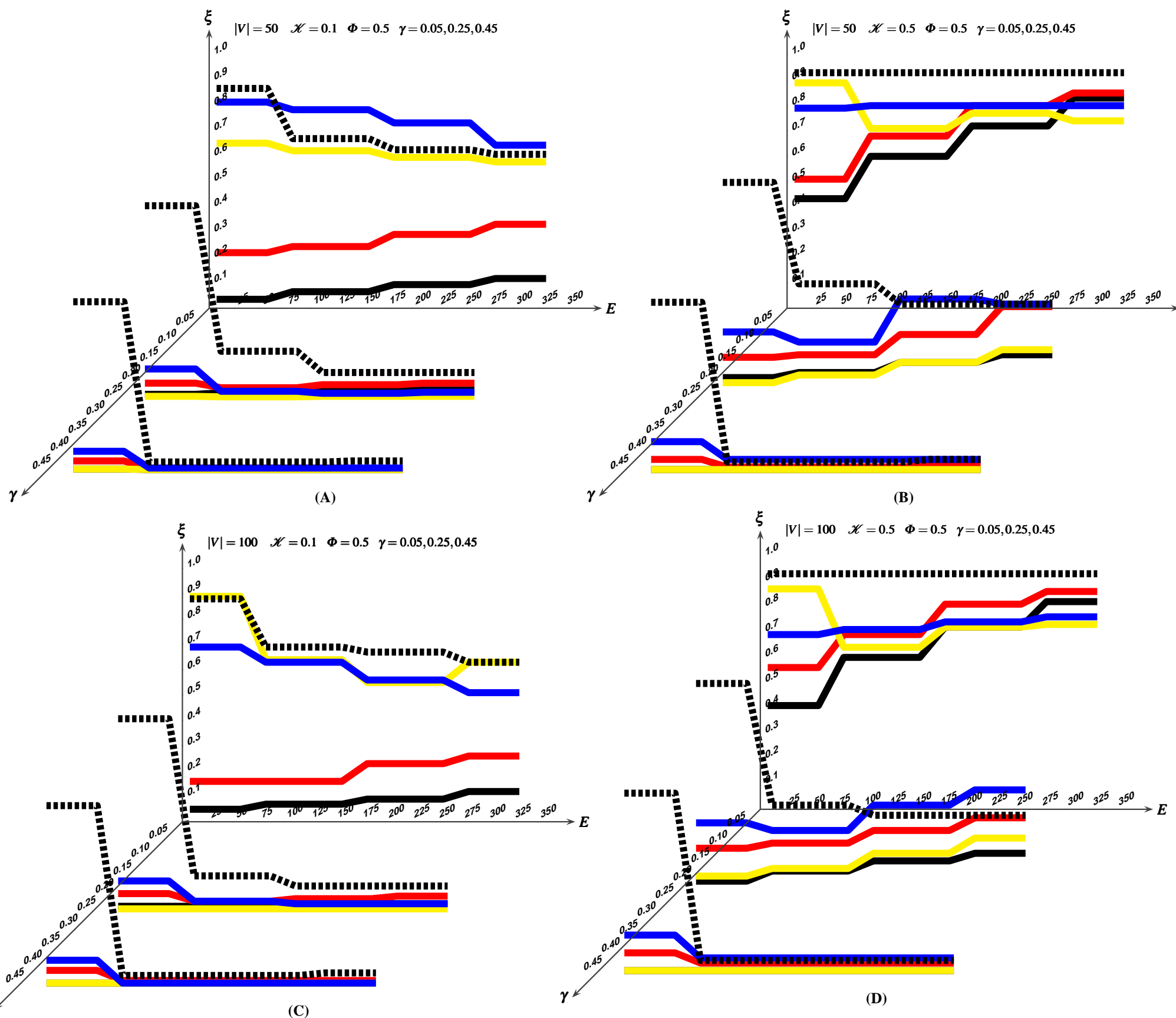

(C)

FIG. S4. Effect of variations of the total external to internal asset ratio $E / I$ on the vulnerability index $\xi$ for homogeneous networks. Lower values of $\xi$ imply higher global stability of a network. 


\section{STABILITY OF FINANCIAL NETWORKS}

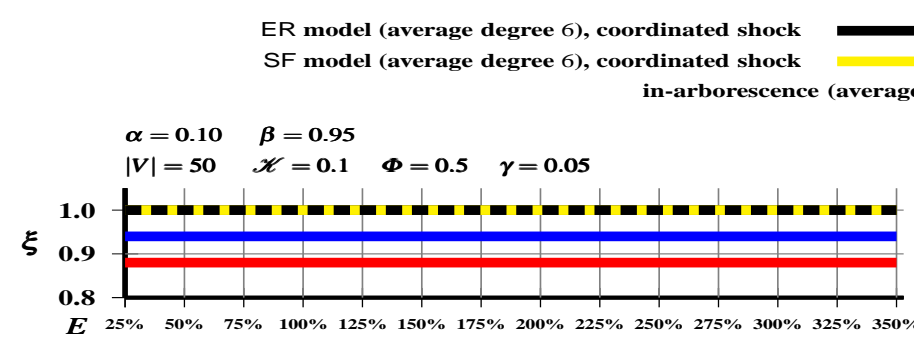

(as \% of $I$ )
(A)

ER model (average degree 3), coordinated shock

SF model (average degree 3 ), coordinated shock legree $\approx 1)$, coordinated shock $\square=\square$

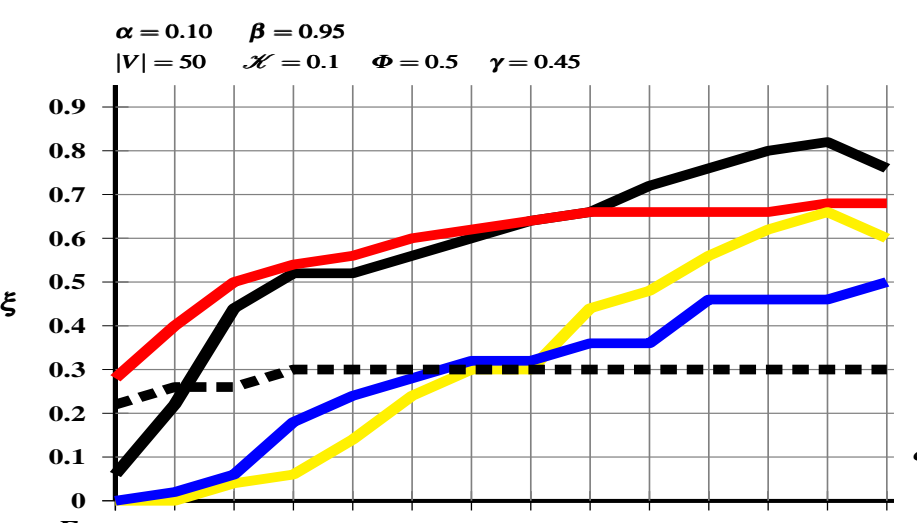

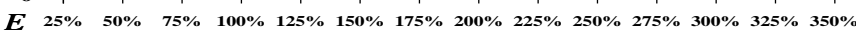
(as \% of $I$ )

(C)

$\xi$

$$
\begin{array}{llll}
\alpha=0.10 & \beta=0.95 & & \\
|V|=100 & \mathscr{K}=0.5 & \Phi=0.5 & \gamma=0.05
\end{array}
$$

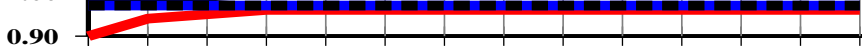

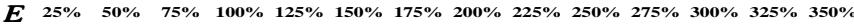
(as \% of $I$ )

(F)

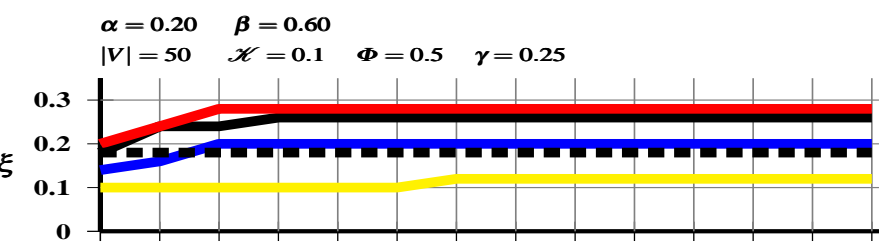

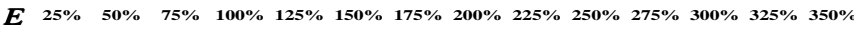
(as \% of $I$ )

(H)

$$
\begin{array}{llll}
\alpha=0.20 & \beta=0.60 & & \\
|V|=50 & \mathscr{K}=0.1 & \Phi=0.5 & \gamma=0.45
\end{array}
$$

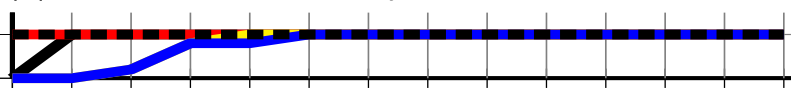

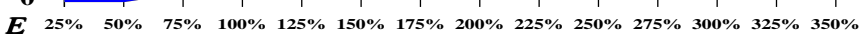
(as \% of $I$ )

(I)

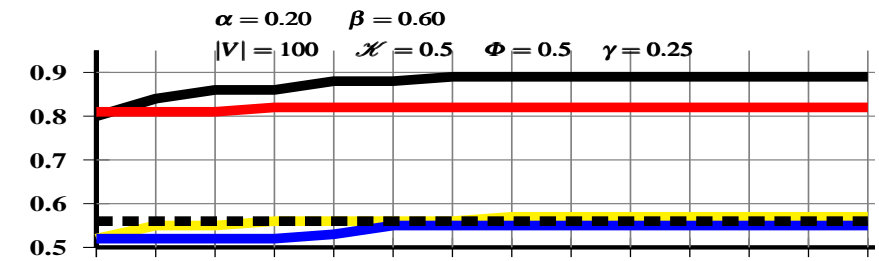

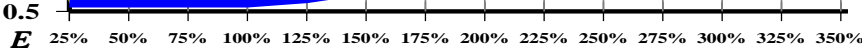
(as \% of $I$ )

(K)

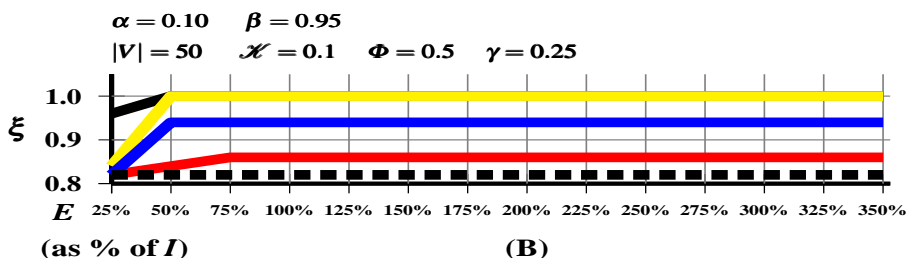

(as \% of $I$ )

(B)

$$
\begin{array}{cccc}
\alpha=0.10 & \beta=0.95 \\
|V|=100 & \mathscr{K}=0.1 \quad \Phi=0.5 \quad \gamma=0.25
\end{array}
$$

1.0 L

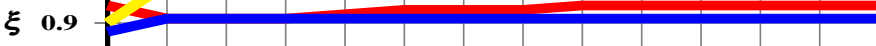

0.8

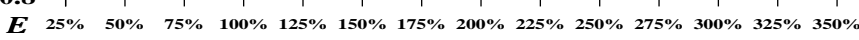
(as \% of $I$ )

(D)

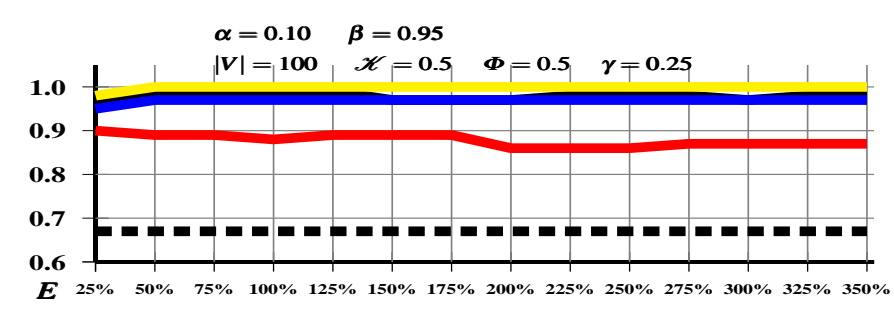

(as \% of $I$ )

(E)

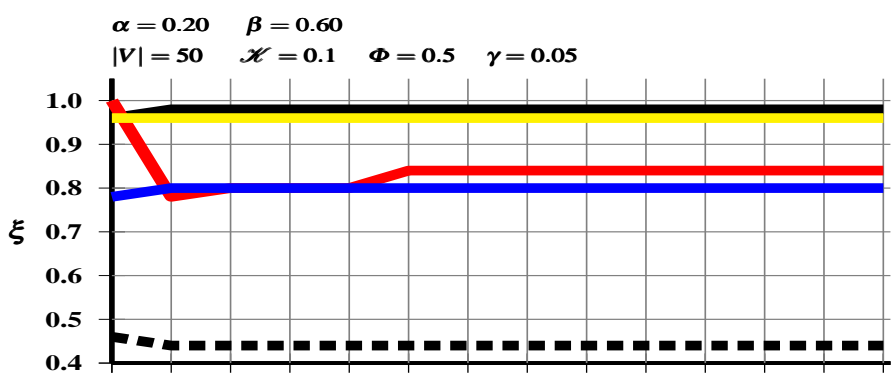

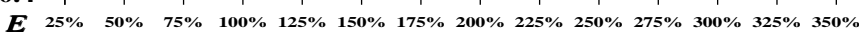
(as \% of $I$ )

(G)

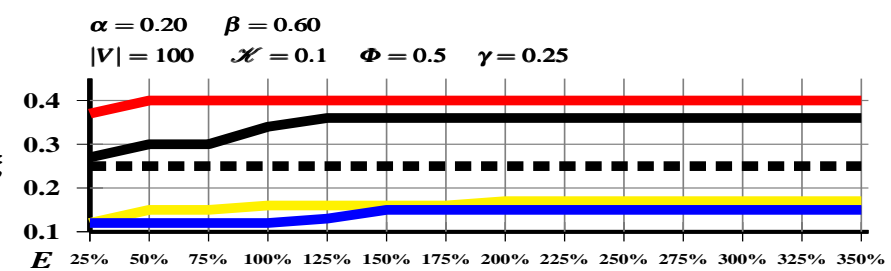

(as \% of $I$ )

(J)

$$
\begin{array}{llll}
\alpha=0.20 & \beta=0.60 & & \\
|V|=100 & \mathscr{K}=0.5 & \Phi=0.5 & \gamma=0.05
\end{array}
$$

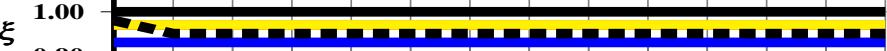

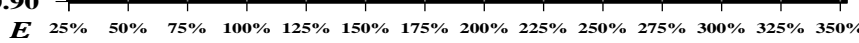
(as \% of $I$ )

(L)

FIG. S5. Effect of variations of the total external to internal asset ratio $E / I$ on the vulnerability index $\xi$ for $(\alpha, \beta)$-heterogeneous networks. Lower values of $\xi$ imply higher global stability of a network. 
DASGUPTA AND KALIGOUNDER

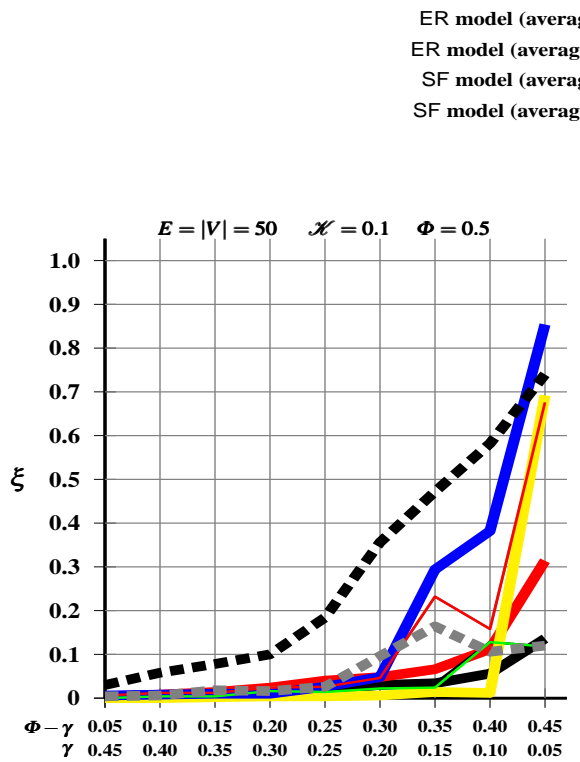

(A)

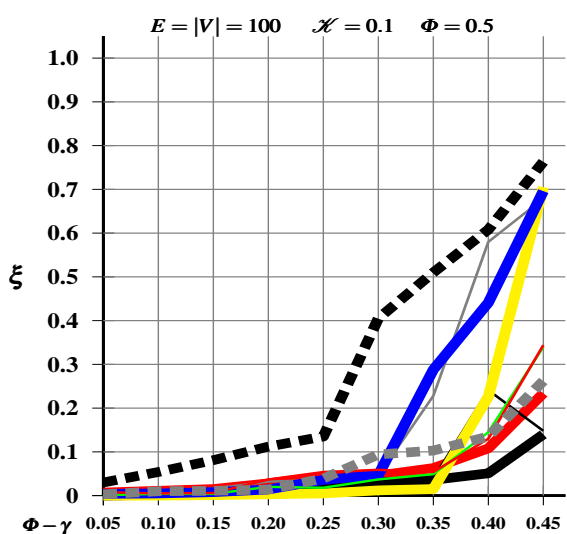

$\begin{array}{rlllllllll}\Phi-\gamma & 0.05 & 0.10 & 0.15 & 0.20 & 0.25 & 0.30 & 0.35 & 0.40 & 0.45 \\ \gamma & 0.45 & 0.40 & 0.35 & 0.30 & 0.25 & 0.20 & 0.15 & 0.10 & 0.05\end{array}$

(D)

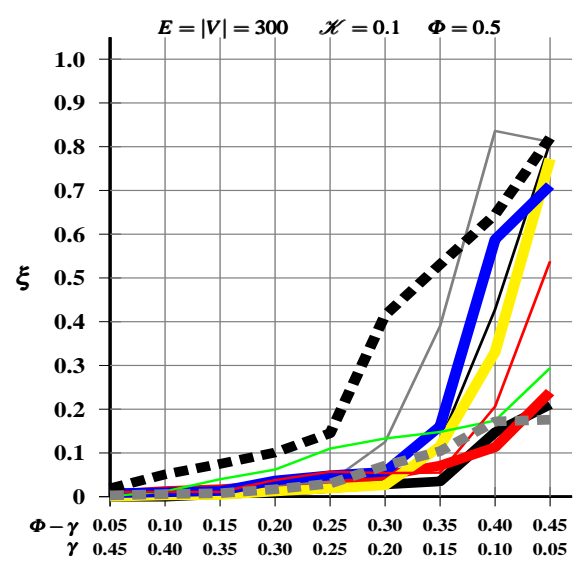

(G)
ER model (average degree 3), coordinated shock ER model (average degree 3), idiosyncratic shock

SF model (average degree 3), coordinated shock

SF model (average degree 3), idiosyncratic shock

in-arborescence (average degree $\approx 1$ ), coordinated shock $= \pm$.

in-arborescence (average degree $\approx 1$ ), idiosyncratic shock $\square \square$

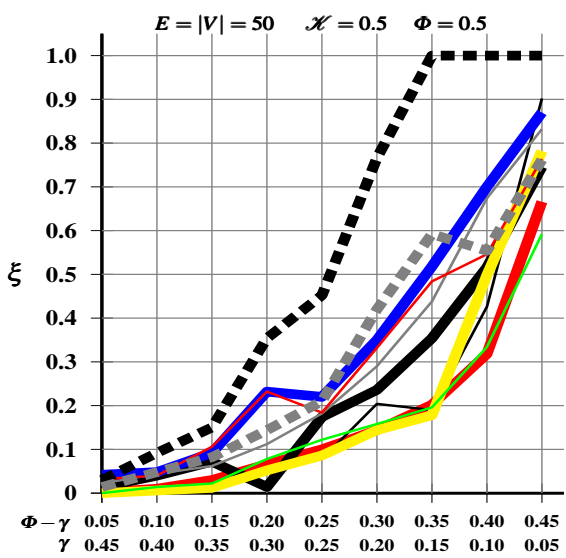

(B)

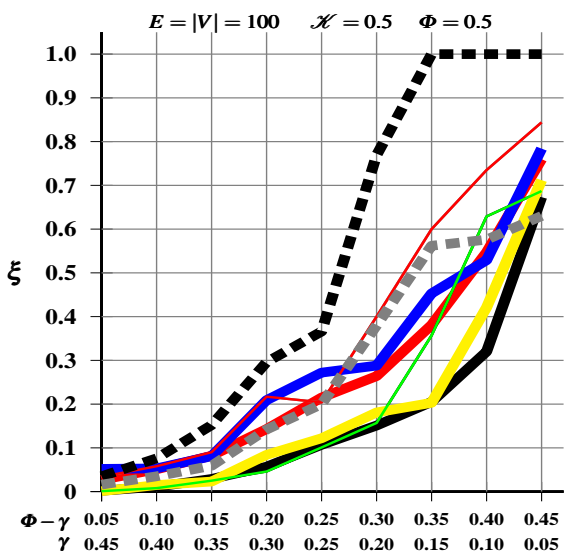

(E)

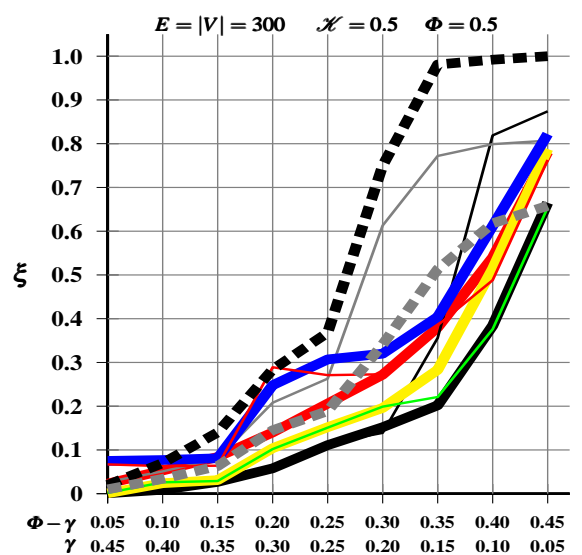

(H)

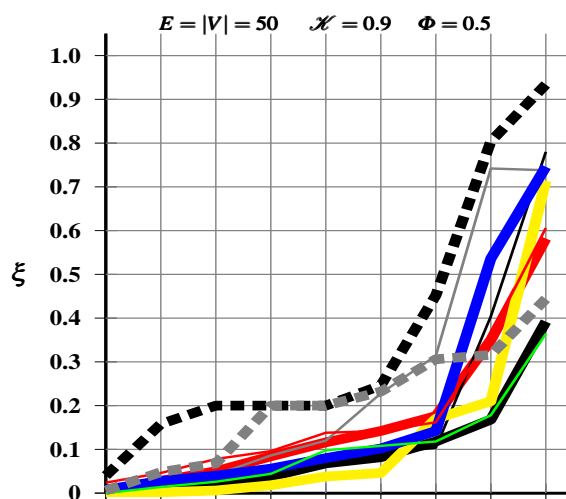

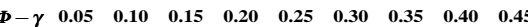
$\begin{array}{lllllllllll}\gamma & 0.45 & 0.40 & 0.35 & 0.30 & 0.25 & 0.20 & 0.15 & 0.10 & 0.05\end{array}$

(C)

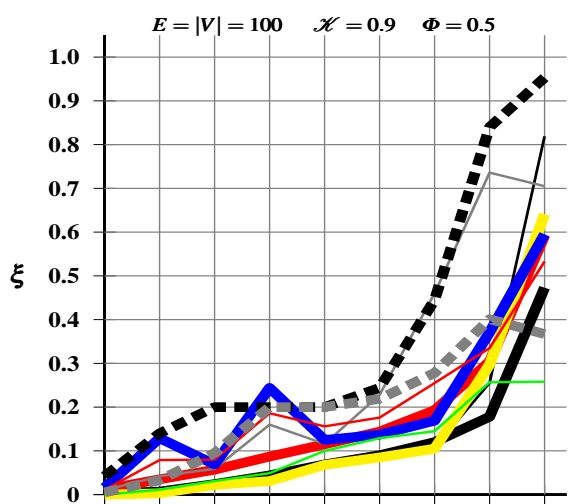

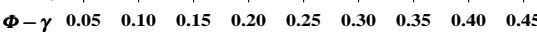
$\begin{array}{llllllllll}\gamma & 0.45 & 0.40 & 0.35 & 0.30 & 0.25 & 0.20 & 0.15 & 0.10 & 0.05\end{array}$

(F)

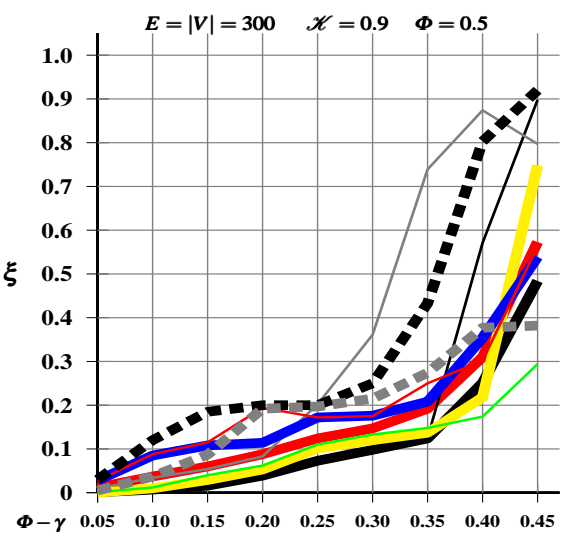

$\begin{array}{rlllllllll}\Phi-\gamma & 0.05 & 0.10 & 0.15 & 0.20 & 0.25 & 0.30 & 0.35 & 0.40 & 0.45 \\ \gamma & 0.45 & 0.40 & 0.35 & 0.30 & 0.25 & 0.20 & 0.15 & 0.10 & 0.05\end{array}$

(I)

FIG. S6. Effect of variations of equity to asset ratio (with respect to shock) on the vulnerability index $\xi$ for homogeneous networks. Lower values of $\xi$ imply higher global stability of a network. 


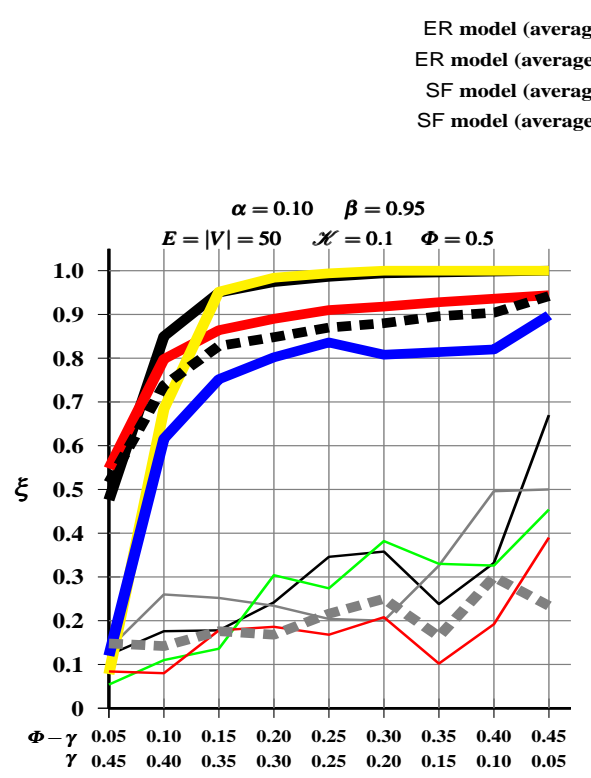

(A)

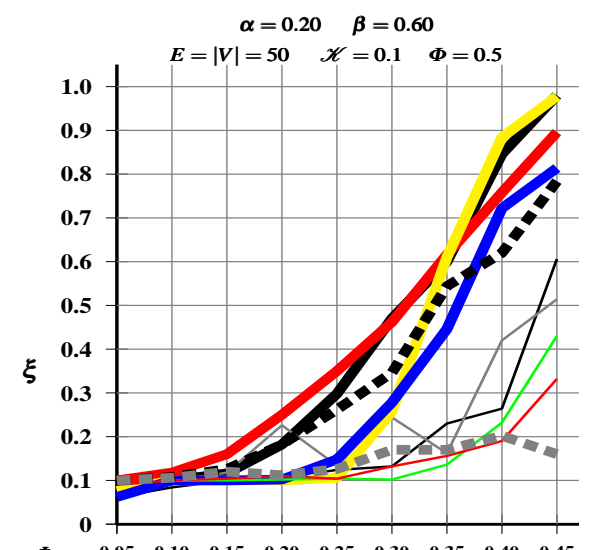

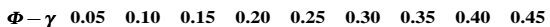
$\begin{array}{llllllllll}\gamma & 0.45 & 0.40 & 0.35 & 0.30 & 0.25 & 0.20 & 0.15 & 0.10 & 0.05\end{array}$

(D)

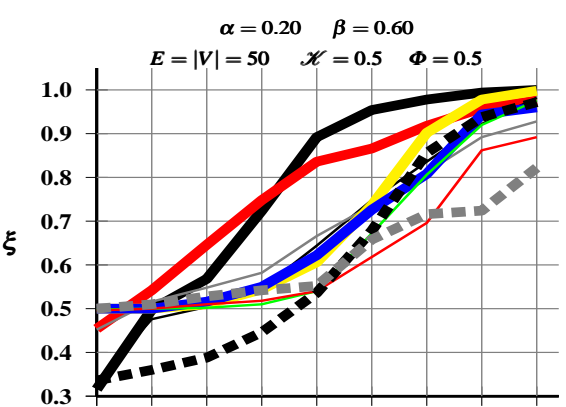

$\begin{array}{llllllllll}\Phi-\gamma & 0.05 & 0.10 & 0.15 & 0.20 & 0.25 & 0.30 & 0.35 & 0.40 & 0.45\end{array}$ $\begin{array}{llllllllll}\gamma & 0.45 & 0.40 & 0.35 & 0.30 & 0.25 & 0.20 & 0.15 & 0.10 & 0.05\end{array}$

(E)

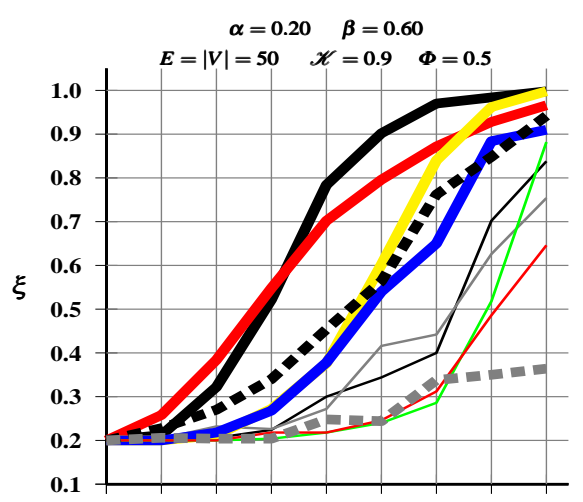

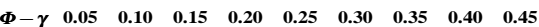
$\begin{array}{llllllllll}\gamma & 0.45 & 0.40 & 0.35 & 0.30 & 0.25 & 0.20 & 0.15 & 0.10 & 0.05\end{array}$

(F)

FIG. S7. Effect of variations of equity to asset ratio (with respect to shock) on the vulnerability index $\xi$ for $(\alpha, \beta)$-heterogeneous networks. Lower values of $\xi$ imply higher global stability of a network. 
DASGUPTA AND KALIGOUNDER

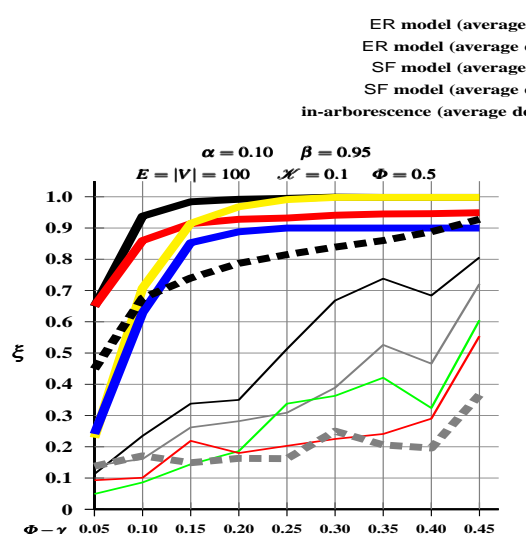

$\begin{array}{rrrrrrrrrr}\Phi-\gamma & 0.05 & 0.10 & 0.15 & 0.20 & 0.25 & 0.30 & 0.35 & 0.40 & 0.45 \\ \gamma & 0.45 & 0.40 & 0.35 & 0.30 & 0.25 & 0.20 & 0.15 & 0.10 & 0.05\end{array}$ (A)

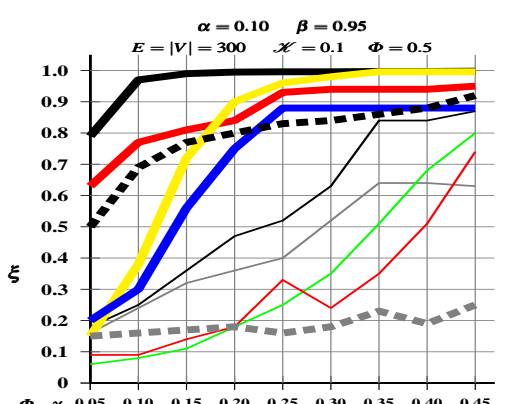

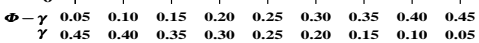

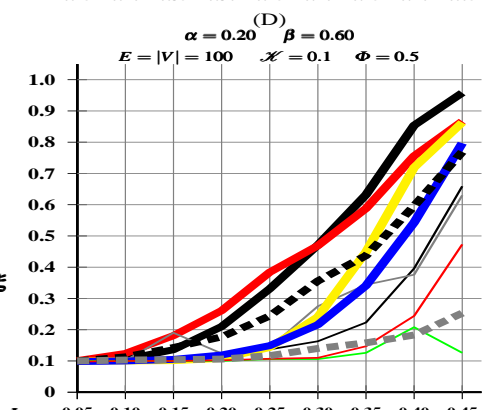

$\begin{array}{rrrrrrrrrr}\Phi-\gamma \gamma & 0.05 & 0.10 & 0.15 & 0.20 & 0.25 & 0.30 & 0.35 & 0.40 & 0.45 \\ \gamma & 0.45 & 0.40 & 0.35 & 0.30 & 0.25 & 0.20 & 0.15 & 0.10 & 0.05\end{array}$ (G)

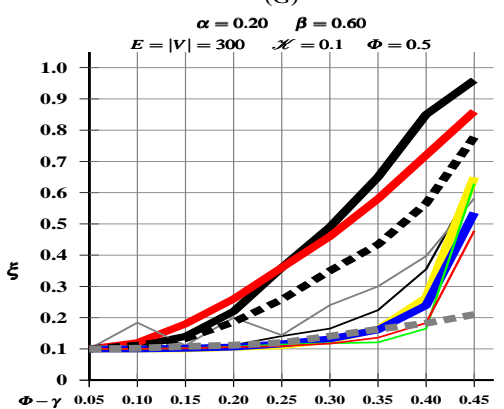

$\begin{array}{cccccccccc}\boldsymbol{\gamma} & 0.05 & 0.10 & 0.15 & 0.20 & 0.25 & 0.30 & 0.35 & 0.40 & 0.45 \\ \gamma & 0.45 & 0.40 & 0.35 & 0.30 & 0.25 & 0.20 & 0.15 & 0.10 & 0.05\end{array}$
ER model (average degree 3), coordinated shock $R$ model (average degree 3), idiosyncratic shock SF model (average degree 3), coordinated shock in-arborescence (average degree $\approx 1$ ), idiosyncratic shock $-\ldots$ $\alpha=0.10 \quad \beta=0.95$

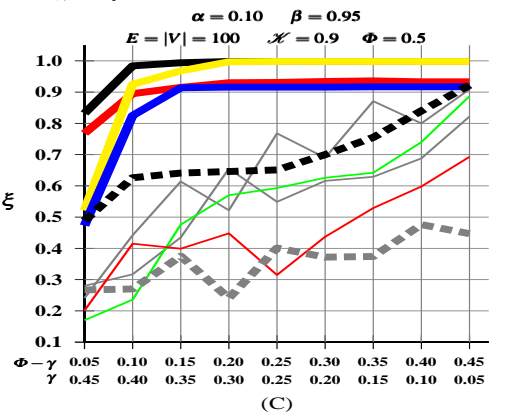
(C)

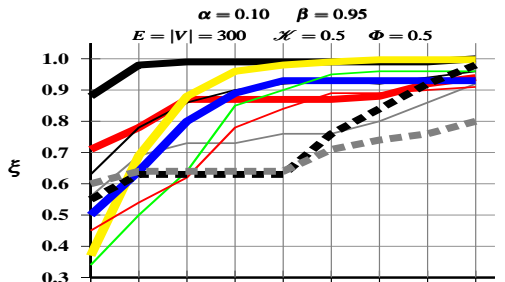

$\begin{array}{rrrrrrrrrr}\Phi-\gamma & 0.05 & 0.10 & 0.15 & 0.20 & 0.25 & 0.30 & 0.35 & 0.40 & 0.45 \\ \gamma & 0.45 & 0.40 & 0.35 & 0.30 & 0.25 & 0.20 & 0.15 & 0.10 & 0.05\end{array}$ (E)
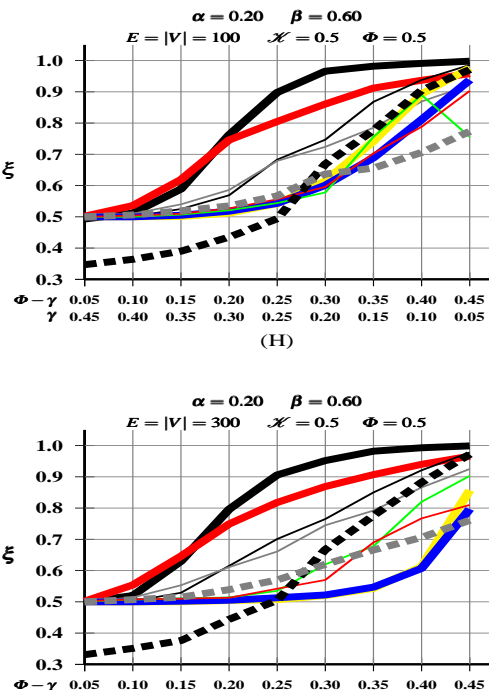

$\begin{array}{rrrrrrrrrr}\Phi-\gamma & 0.05 & 0.10 & 0.15 & 0.20 & 0.25 & 0.30 & 0.35 & 0.40 & 0.45 \\ \gamma & 0.45 & 0.40 & 0.35 & 0.30 & 0.25 & 0.20 & 0.15 & 0.10 & 0.05\end{array}$ (K)

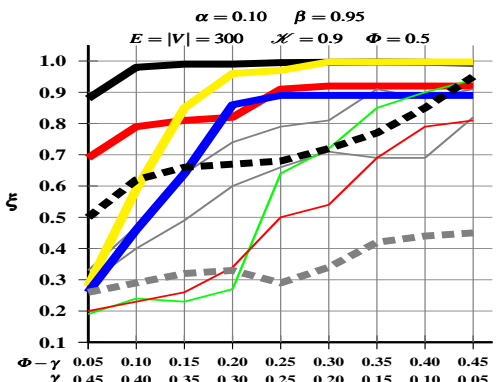

(F)

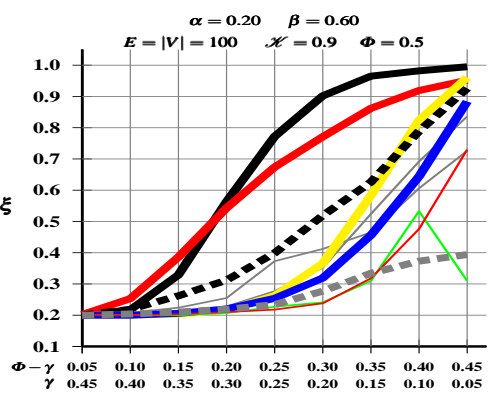

(I)

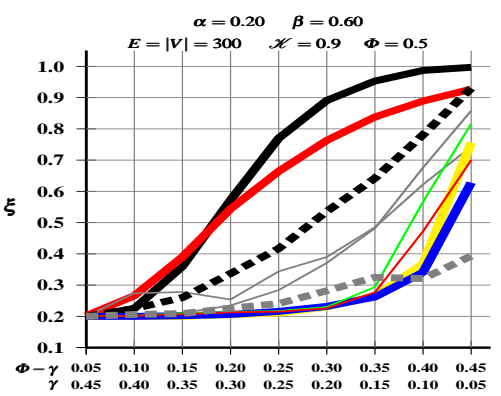

(L)

(J)

FIG. S8. Effect of variations of equity to asset ratio (with respect to shock) on the vulnerability index $\xi$ for $(\alpha, \beta)$-heterogeneous networks. Lower values of $\xi$ imply higher global stability of a network. 
ER model (average degree 6), coordinated shock - $\quad$ ER model (average degree 3), coordinated shock

ER model (average degree 6), idiosyncratic shock — $\quad$ ER model

SF model (average degree 6), coordinated shock

SF model (average degree 3), coordinated shock

SF model (average degree 6), idiosyncratic shock

SF model (average degree 3), idiosyncratic shock -

in-arborescence (average degree $\approx 1$ ), coordinated shock $\mathrm{I}$ I I

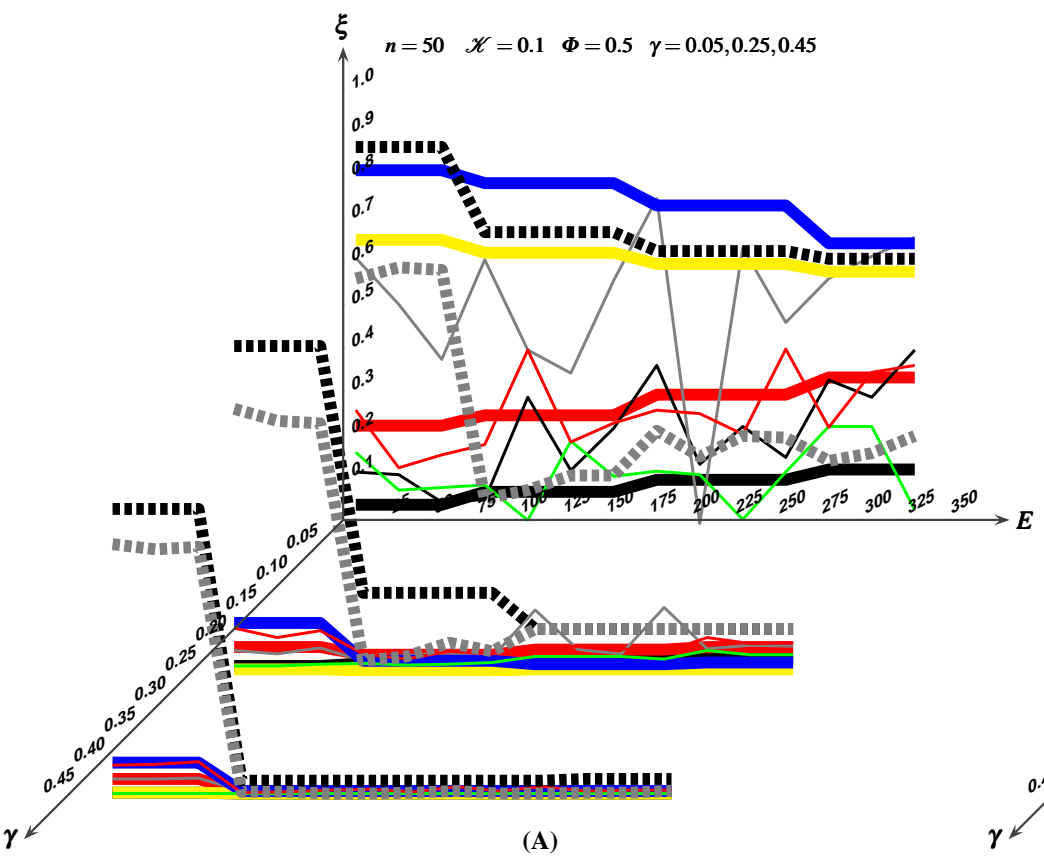

in-arborescence (average degree $\approx 1$ ), idiosyncratic shock $\|$ u

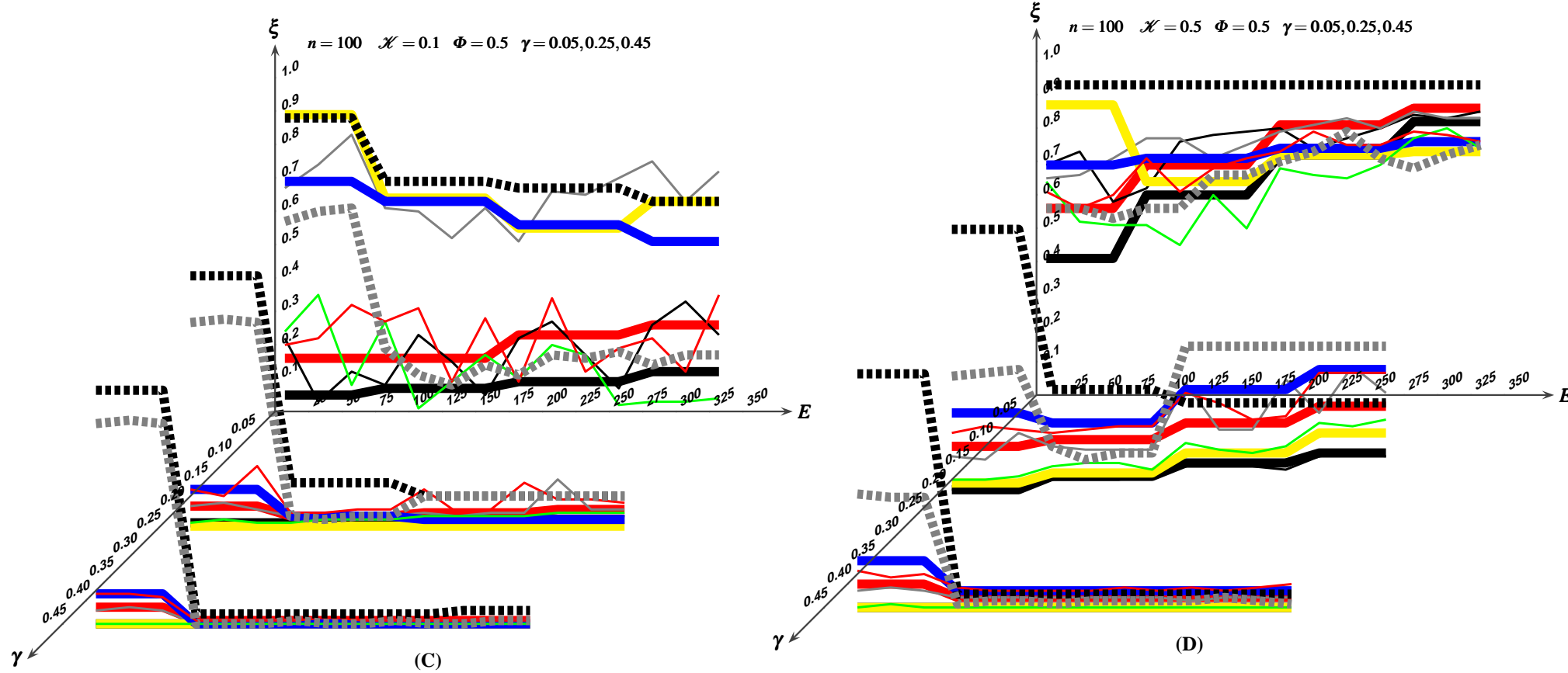

FIG. S9. Effect of variations of the total external to internal asset ratio $E / I$ on the vulnerability index $\xi$ for homogeneous networks.

Lower values of $\xi$ imply higher global stability of a network. 
DASGUPTA AND KALIGOUNDER

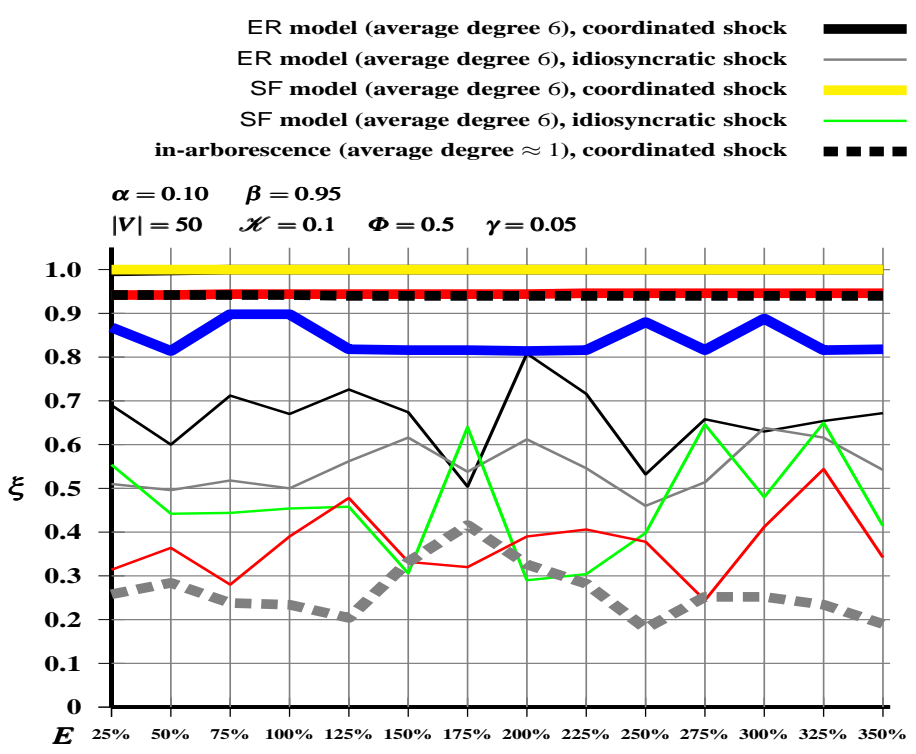

(as \% of $I$ )
(A)
ER model (average degree 3), coordinated shock
ER model (average degree 3), idiosyncratic shock
SF model (average degree 3), coordinated shock
SF model (average degree 3 ), idiosyncratic shock

in-arborescence (average degree $\approx 1$ ), idiosyncratic shock $\quad=\square$ $\alpha=0.10 \quad \beta=0.95$

$|V|=50 \quad \mathscr{K}=0.1 \quad \Phi=0.5 \quad \gamma=0.25$

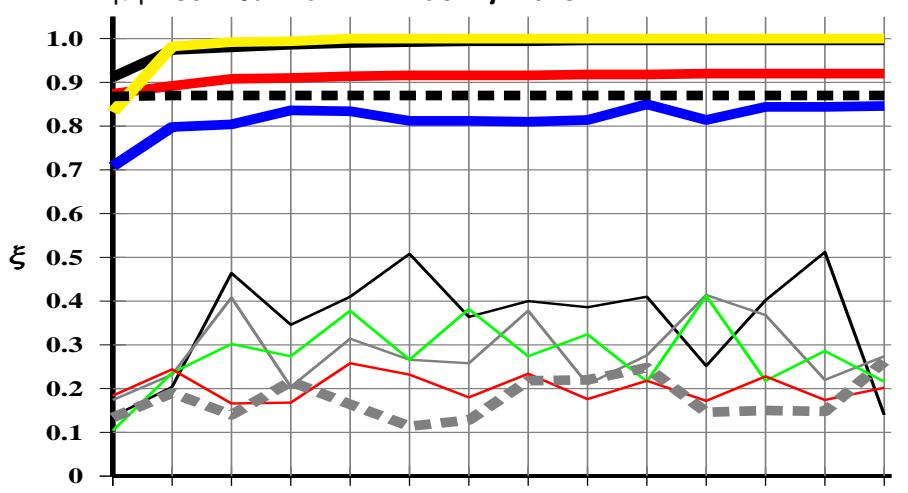

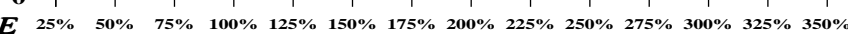
(as \% of $I$ )

(B)

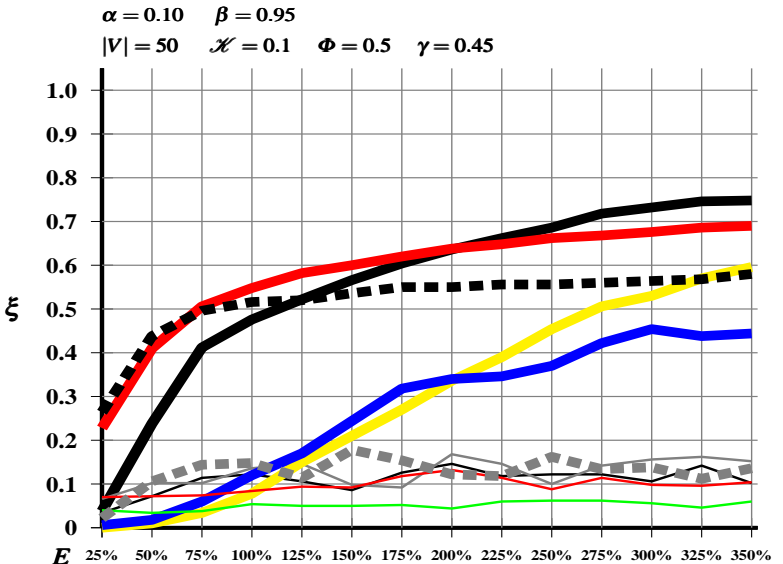

(as \% of $I$ )

(C)

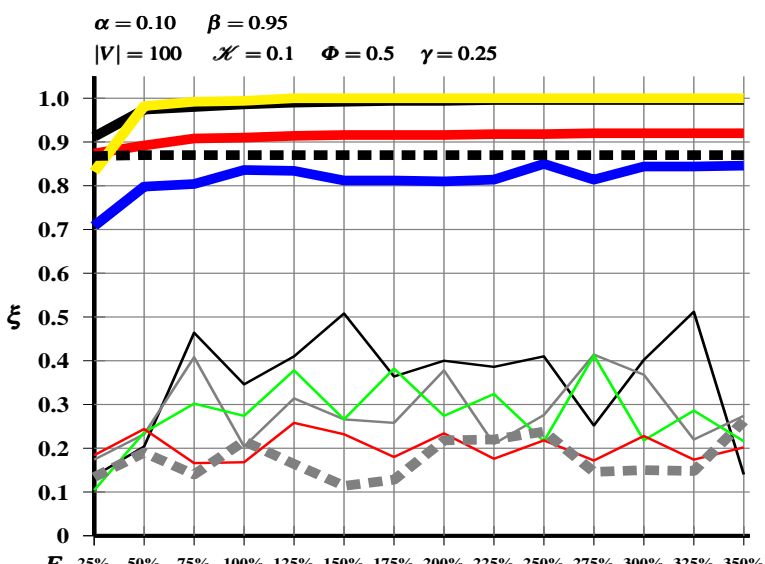

(as \% of $I$ )

(D)

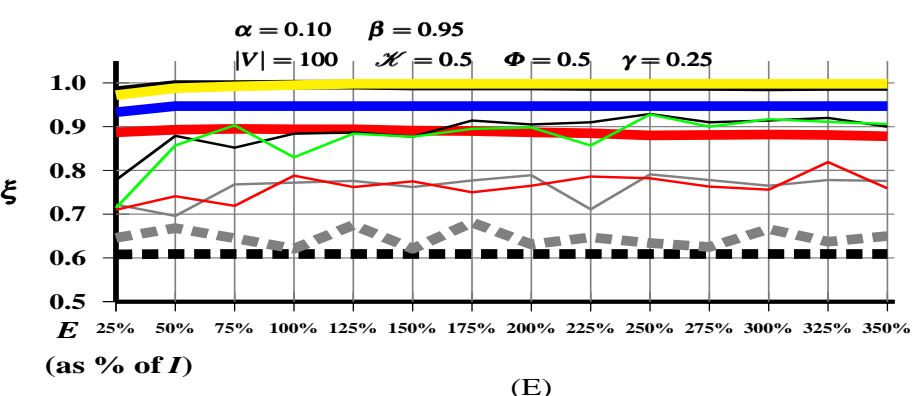

(E)

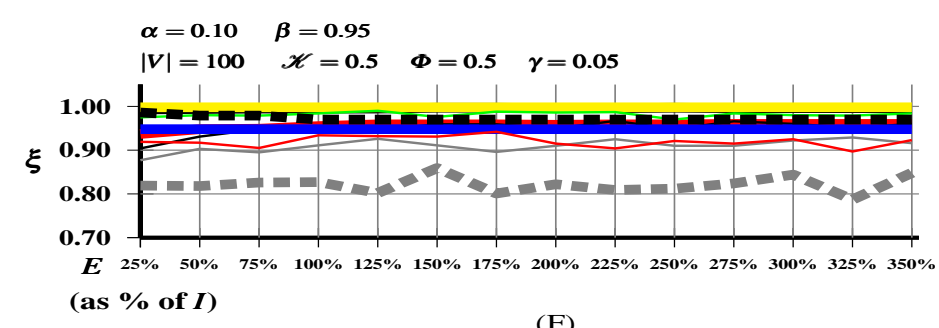

(F)

FIG. S10. Effect of variations of the total external to internal asset ratio $E / I$ on the vulnerability index $\xi$ for $(\alpha, \beta)$-heterogeneous networks. Lower values of $\xi$ imply higher global stability of a network. 
ER model (average degree 6), coordinated shock ER model (average degree 6), idiosyncratic shock SF model (average degree 6), coordinated shock

SF model (average degree 6), idiosyncratic shock in-arborescence (average degree $\approx 1$ ), coordinated shock

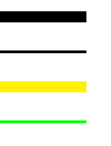

$\mathbf{a} \mathbf{\square}$
ER model (average degree 3), coordinated shock ER model (average degree 3), idiosyncratic shock SF model (average degree 3 ), coordinated shock SF model (average degree 3 ), idiosyncratic shock in-arborescence (average degree $\approx 1$ ), idiosyncratic shock $\square$

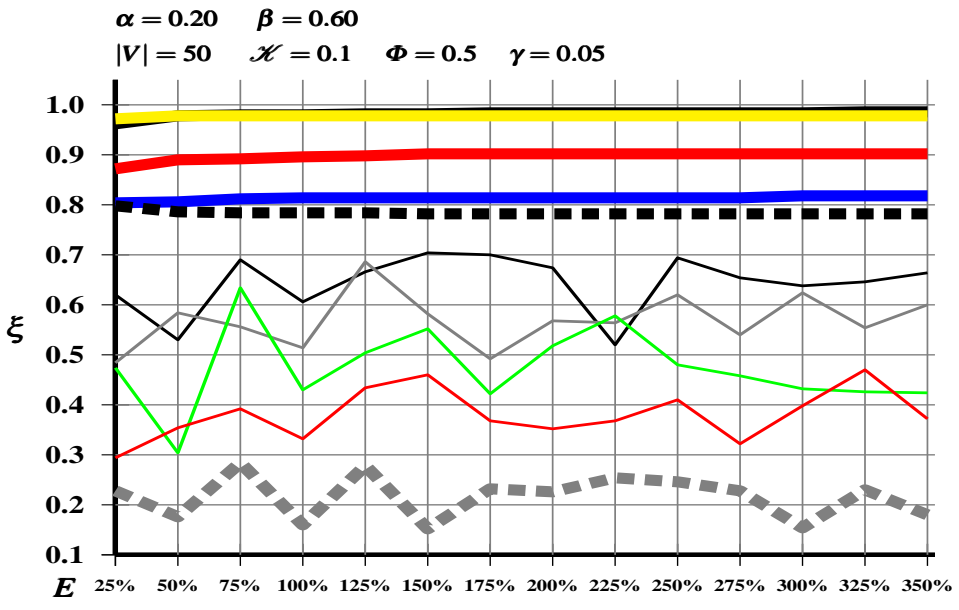

(as \% of $I$ )

(A)

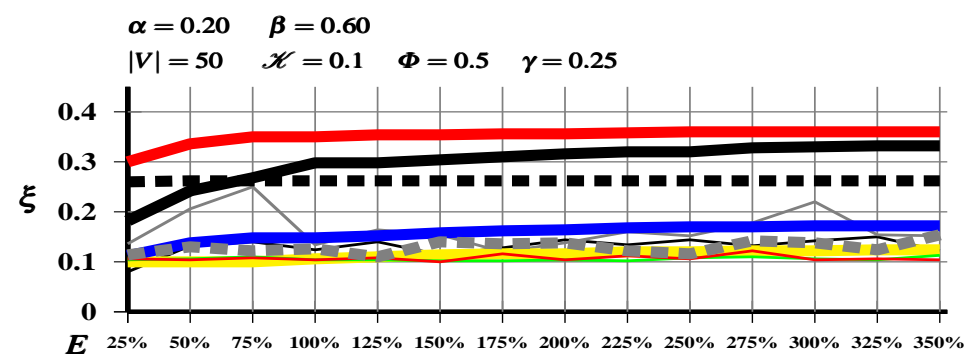

(as \% of I)

(B)

$$
\begin{array}{llll}
\alpha=0.20 & \beta=0.60 & \\
|V|=50 & \mathscr{K}=0.1 \quad \Phi=0.5 & \gamma=0.45
\end{array}
$$

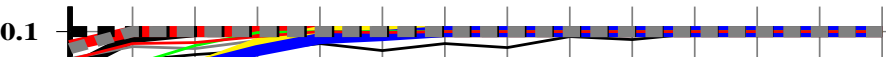
0

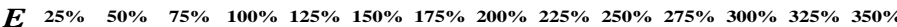
(as \% of $I$ )

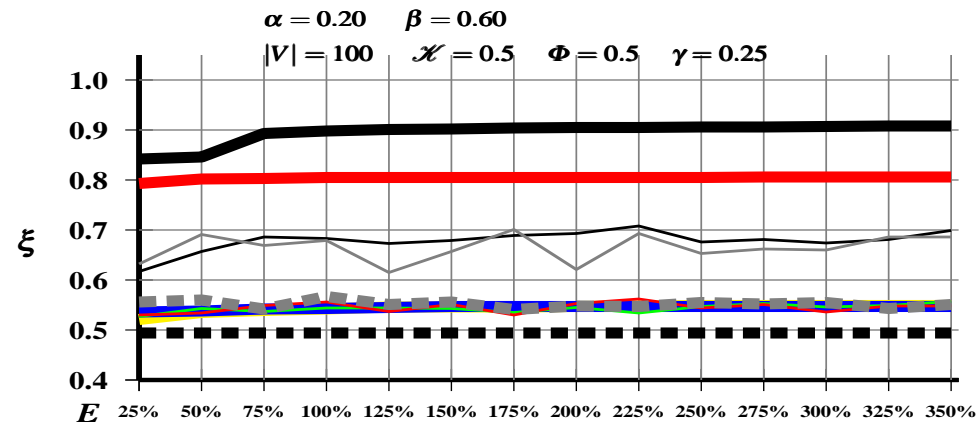

(as \% of $I$ )

(E)

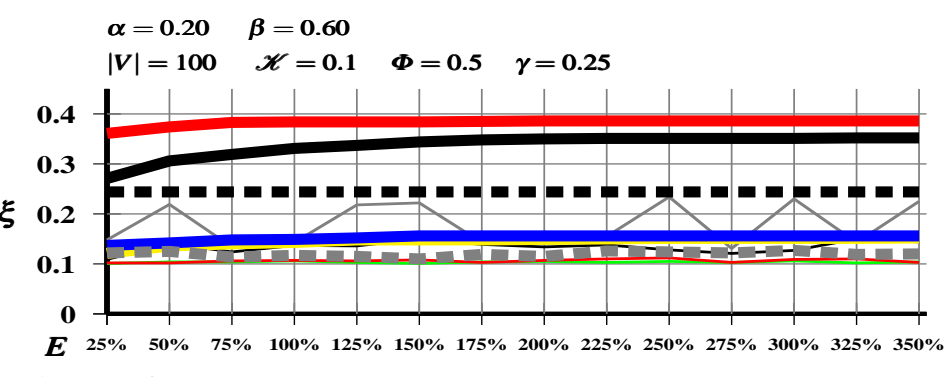

(as \% of I)

$$
\begin{array}{lll}
\alpha=0.20 & \beta=0.60 \\
|V|=100 & \mathscr{K}=0.5 \quad \Phi=0.5 \quad \gamma=0.05
\end{array}
$$

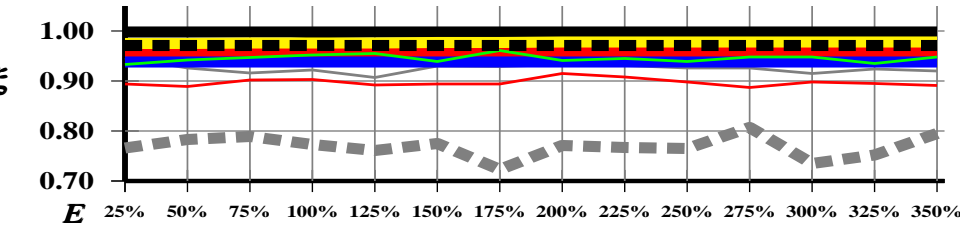

(as \% of $I)$

FIG. S11. Effect of variations of the total external to internal asset ratio $\frac{\mathscr{E}}{\mathscr{J}}$ on the vulnerability index $\xi$ for $(\alpha, \beta)$-heterogeneous networks. Lower values of $\xi$ imply higher global stability of a network. 